\title{
Deforming Composite Grids for Solving Fluid Structure Problems
}

\author{
Jeffrey W. Banks ${ }^{\mathrm{a}, 1}$, William D. Henshaw ${ }^{\mathrm{a}, 1, *}$, Donald W. Schwendeman ${ }^{\mathrm{b}, 2}$ \\ ${ }^{a}$ Centre for Applied Scientific Computing, Lawrence Livermore National Laboratory, Livermore, CA 94551, USA \\ ${ }^{b}$ Department of Mathematical Sciences, Rensselaer Polytechnic Institute, Troy, NY 12180, USA
}

\begin{abstract}
We describe a mixed Eulerian-Lagrangian approach for solving fluid-structure interaction (FSI) problems. The technique, which uses deforming composite grids (DCG), is applied to FSI problems that couple high speed compressible flow with elastic solids. The fluid and solid domains are discretized with composite overlapping grids. Curvilinear grids are aligned with each interface and these grids deform as the interface evolves. The majority of grid points in the fluid domain generally belong to background Cartesian grids which do not move during a simulation. The FSI-DCG approach allows large displacements of the interfaces while retaining high quality grids. Efficiency is obtained through the use of structured grids and Cartesian grids. The governing equations in the fluid and solid domains are evolved in a partitioned approach. We solve the compressible Euler equations in the fluid domains using a high-order Godunov finite-volume scheme. We solve the linear elastodynamic equations in the solid domains using a second-order upwind scheme. We develop interface approximations based on the solution of a fluid-solid Riemann problem that results in a stable scheme even for the difficult case of light solids coupled to heavy fluids. The FSI-DCG approach is verified for three problems with known solutions, an elastic-piston problem, the superseismic shock problem and a deforming diffuser. In addition, a self convergence study is performed for an elastic shock hitting a fluid filled cavity. The overall FSI-DCG scheme is shown to be second-order accurate in the max-norm for smooth solutions, and robust and stable for problems with discontinuous solutions for a wide range of constitutive parameters.
\end{abstract}

Keywords: fluid-structure interactions, overlapping grids, gas dynamics, elasticity, interface stability, added-mass instability

${ }^{*}$ Corresponding author. Mailing address: Centre for Applied Scientific Computing, L-422, Lawrence Livermore National Laboratory, Livermore, CA 94551, USA. Phone: 925-423-2697. Fax: 925-424-2477.

Email addresses: banks20@1lnl.gov (Jeffrey W. Banks), henshaw1@llnl.gov (William D. Henshaw), schwed@rpi.edu (Donald W. Schwendeman)

${ }^{1}$ This work was performed under the auspices of the U.S. Department of Energy (DOE) by Lawrence Livermore National Laboratory under Contract DE-AC52-07NA27344 and by DOE contracts from the ASCR Applied Math Program.

${ }^{2}$ This research was supported by Lawrence Livermore National Laboratory under subcontract B548468, and by the National Science Foundation under grants DMS-0532160 and DMS-1016188. 


\section{Contents}

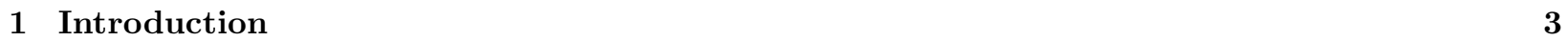

2 Nomenclature $\quad 4$

\begin{tabular}{llr}
\hline 3 & Governing equations & 4
\end{tabular}

4 One-dimensional elastic piston $\quad 5$

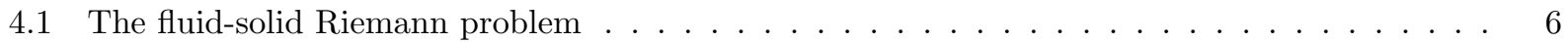

4.1 .1 The linearized fluid-solid Riemann problem $\ldots \ldots \ldots \ldots$. . . . . . . . . . 6

4.1 .2 The nonlinear fluid-solid Riemann problem . . . . . . . . . . . . . . . . . . 8

4.2 The FSI time-stepping algorithm and discrete interface approximations $\ldots \ldots \ldots$

$4.2 .1 \quad$ Interior Stage . . . . . . . . . . . . . . . . . . . . . . . . . 9

4.2 .2 Interface Stage . . . . . . . . . . . . . . . . . . . . . . . . . . 9

$4.2 .3 \quad$ Ghost Stage . . . . . . . . . . . . . . . . . . . . . . . . . . . . . . . . 10

4.2 .4 Limited extrapolation . . . . . . . . . . . . . . . . . . . . . . . 11

4.3 Numerical results for the one-dimensional elastic-piston $\ldots \ldots \ldots \ldots \ldots$

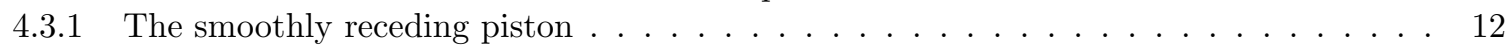

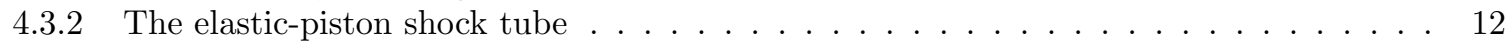

5 The FSI-DCG numerical approach for initial-boundary-value problems $\quad 14$

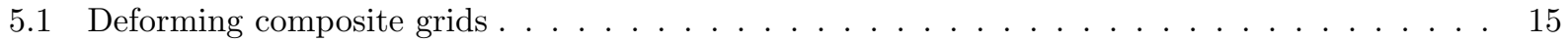

5.2 Discretization of the fluid and solid governing equations $\ldots \ldots \ldots \ldots \ldots$

5.3 Multi-domain time-stepping algorithm $\ldots \ldots \ldots \ldots \ldots \ldots \ldots$

6 Numerical results $\quad 19$

6.1 The two-dimensional elastic piston $\ldots \ldots \ldots \ldots \ldots \ldots \ldots$

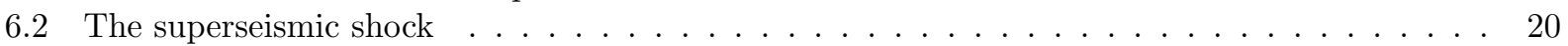

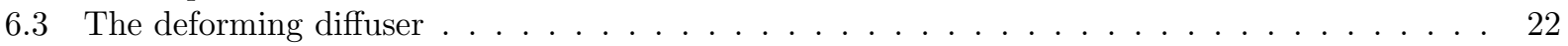

6.4 An elastic shock impacting a deformable fluid cylinder . . . . . . . . . . . . . . . 25

6.5 A shock impacting two deformable sticks . . . . . . . . . . . . . . . . . 27

$\begin{array}{llr}7 & \text { Conclusions } & 30\end{array}$

$\begin{array}{llll}\text { Appendix A } & \text { Solution of the receding elastic-piston problem } & 30\end{array}$

$\begin{array}{llll}\text { Appendix B } & \text { Solution of the elastic-piston shock-tube problem } & 32\end{array}$

$\begin{array}{llll}\text { Appendix } & \text { C } & \text { Solution of the superseismic-shock problem } & 32\end{array}$ 


\section{Introduction}

Fluid-structure interaction (FSI) problems are multi-domain problems involving moving and deforming solids that are coupled to a neighboring fluid or gas. These problems are important in many fields of engineering and applied science and are often computationally challenging. Examples of FSI problems include the modeling of flow-induced vibrations of structures (aircraft, undersea cables, wind turbines, buildings), parachutes and airbags, blood flow in veins and arteries, acoustic lenses, thermal expansion in nuclear reactor cores, shock-structure interactions (blast effects), and weapons effects, to name just a few.

FSI is an active field of research and there are a wide variety of numerical techniques that have been developed for simulating problems in this area. These techniques include Eulerian, Lagrangian, Arbitrary Lagrangian-Eulerian (ALE) [1, 2, 3, 4], immersed boundary [5, 6], embedded boundary [7], level-set [8, 9], interface tracking [10, 11], and distributed-Lagrange-multiplier/fictitious-domain methods [12]. Numerical approximations based on finite-element [13], finite-volume [14, 15] and discontinuous Galerkin methods [16], among others, have been developed. FSI simulations can be extremely challenging for a number of reasons. They can require the coupling of multiple complex physics regimes involving disparate spatial and temporal scales. In addition, developing accurate and stable numerical approximations for the coupling conditions between different physical domains is often nontrivial. In particular, the simulation of light solids has proven to be difficult for many conventional algorithms. This difficulty is often attributed to the so-called added mass effect whereby the additional force required to push a light body through a heavy fluid (as compared to pushing the body through a vacuum) can be interpreted as the body having an additional mass (tensor) [17]. A monolithic approach, forming a fully coupled implicit system for fluids and solids, is often used to avoid stability problems associated with light solids. Monolithic solvers, however, can be expensive and require the development of new forms of preconditioners to solve the coupled FSI equations. In this article, we present an interface approximation for partitioned solution algorithms that remains stable for a wide range of FSI regimes, including those involving light solids.

Another key challenge for FSI simulations is the accurate representation of interfaces and the need to maintain high-quality grids even under large displacements and rotations. Fast algorithms for grid regeneration at each time step are particularly challenging. The different approaches to solving FSI problems all have their strengths and weaknesses in terms of range of applicability, flexibility, accuracy and computational performance. Approaches based on block-structured grids, for example, can be efficient and accurate, but they have difficulty treating general motions of solid bodies. Unstructured-mesh algorithms, on the other hand, can be flexible at representing the geometry of an FSI problem, but can be computationally expensive and present challenges in terms of mesh quality as the geometry evolves with time.

In this article we describe a mixed Eulerian-Lagrangian approach for solving FSI problems. The technique is based on the use of deforming composite grids (DCG) to locally represent interfaces with deforming curvilinear grids that overlap other static grids. The FSI-DCG approach allows large displacements of interfaces while retaining high-quality grids and is thus especially effective for problems where solids move large distances and undergo large rotations. Highly distorted interfaces, however, would require the use of a locally fine mesh since the interface is represented with a body-fitted grid. Efficiency is achieved through the use of structured and Cartesian grids along with fast moving-grid generation algorithms. This FSIDCG methodology builds upon previous work in the approximation of partial differential equations (PDEs) using the overlapping grid technique. Overlapping grids have been used to solve a wide variety of problems, primarily in the fields of aerodynamics and fluid dynamics, but more recently they have also been applied to electromagnetics [18] and solid mechanics [19]. Overlapping grids were recognized early on to be a useful technique for treating rigid moving bodies, such as aircraft store separation [20], and have subsequently been applied to many other moving-grid aerodynamic applications, see for example [21, 22, 23, 24, 25].

The FSI-DCG technique is a general approach which extends the overlapping-grid technology to a wide class of multi-domain problems with evolving and deforming interfaces. However, in the current context we first restrict our attention to coupling compressible ideal gases with linear elastic solids. The algorithms and interface approximations are developed and verified for this important model problem before considering other models for the fluid or solid. An important aspect of this current work is the development of a scheme that is second-order accurate for smooth solutions, robust for shocks, and stable for a wide range of material parameters. In the FSI-DCG approach, the fluid and solid domains are independently discretized with overlapping grids. One or more curvilinear grids are aligned with each fluid-solid interface and these grids deform as the interface evolves. The majority of the grid points in the fluid domain generally remain fixed during the computation, and static grids are used to discretize the fixed reference domains for the solid. The governing equations in the fluid and solid domains are evolved in a partitioned approach (although a coupled 
monolithic approach is also possible). In fluid domains we solve the Euler equations for a compressible inviscid fluid on a (possibly) moving grid using a high-order Godunov finite-volume scheme as described in [22]. In solid domains we solve the elastic wave equation on a fixed reference grid using a second-order upwind scheme as discussed in [19]. The multi-domain time-stepping algorithm we use is an extension of the approach developed in [26] for the case of conjugate heat transfer (coupling incompressible fluid flow and heat transfer in solids).

At a fluid-solid interface of an FSI problem, the normal components of the fluid and solid velocities must match along with the fluid and solid tractions (the traction is the force per unit area on the interface). The standard partitioned approach to fluid-solid interfaces attempts to decouple these two conditions by choosing the interface velocity to be that of the solid, and the interface traction to be that of the fluid. Although this velocity-from-solid/stress-from-fluid (VS/SF) approximation performs well for many situations, it is unstable for case of light solids coupled to heavy fluids (more correctly it is unstable when the ratio of the solid impedance to fluid impedance is too small, see Section 4). Extending the recent work of Banks and Sjögreen [27], we have developed a new fluid-solid interface approximation that is based on the solution of a fluid-solid Riemann problem. The linearized form of this interface approximation determines the interface velocity and traction as an impedance weighted average of the fluid and solid values. It reduces to the standard VS/SF scheme in the limit of very heavy solids but uses a stress-from-solid/velocity-from-fluid (SS/VF) approximation in the limit of very light solids. The stability of the linearized interface scheme for the linearized equations was proved in [27]. The related nonlinear interface scheme described herein also appears to be stable for all ratios of the fluid to solid impedances.

The remaining sections of the paper are organized as follows. Section 2 provides a list of the principal variables and their symbols used in the paper. In Section 3 we present the governing equations, boundary conditions, and interface conditions that define the FSI initial-boundary-value problem under consideration. Section 4 is devoted to a discussion of the one-dimensional elastic-piston FSI problem. The solution to the fluid-solid Riemann problem is presented and a number of exact solutions are derived; these are used later for numerical verification. The FSI-DCG algorithm and interface scheme are described in detail for the elastic-piston, and the resulting numerical approximations are verified. In Section 5.1 we describe the multi-dimensional FSI-DCG approach and give a short overview of the overlapping grid technique. In Section 5.2 we summarize the discrete approximations used to solve the governing equations in the fluid and solid domains, while in Section 5.3 we outline the multi-domain time-stepping algorithm used for solving the coupled FSI problem. Section 6 provides numerical verification of the full two-dimensional FSI-DCG scheme. We begin by verifying the FSI-DCG scheme for three problems with known solutions: the twodimensional elastic-piston problem, the superseismic-shock problem, and a deforming-diffuser problem. We then perform simulations of an elastic shock impacting a fluid-filled cavity to demonstrate the stability of the two-dimensional interface approximations for light and heavy solids. We close Section 6 by considering the problem of a shock impacting two deformable sticks. Concluding remarks are made in Section 7 .

\section{Nomenclature}

In this brief section, we list the principal variables that appear in this article and the symbols used to denote them. Variables associated with the solid are indicated by an over-bar to distinguish them from variables related to the fluid.

$\bar{\rho}, \overline{\mathbf{v}}=\left(\bar{v}_{1}, \bar{v}_{2}\right), \overline{\mathbf{u}}=\left(\bar{u}_{1}, \bar{u}_{2}\right), \overline{\boldsymbol{\sigma}}=\left(\bar{\sigma}_{m n}\right):$ solid density, displacement, velocity and stress tensor.

$(\lambda, \mu), c_{p}, c_{s}$ : Lamé material parameters, p-wave speed, and s-wave speed for the solid.

$\rho, \mathbf{v}=\left(v_{1}, v_{2}\right), p, E:$ fluid density, velocity, pressure and total energy.

$\gamma, a$ : ratio of specific heats and speed of sound in the fluid.

$\Omega_{k}, \mathbf{x}$ : fluid domain $k$, fluid spatial coordinates.

$\bar{\Omega}_{k}, \overline{\mathbf{x}}$ : solid domain $k$, solid reference coordinates.

$\mathcal{G}, G_{g}$ : composite grid, component grid $g$.

$\mathbf{x}=\mathbf{G}(\mathbf{r}, t)$ : mapping (from the unit-square coordinates $\mathbf{r}$ ) for a moving component grid.

\section{Governing equations}

We consider the solution to an FSI initial-boundary-value-problem (IBVP). The domain of interest consists of a set of fluid domains $\left\{\Omega_{k}\right\}, k=1,2, \ldots \mathcal{N}_{f}$, and a set of solid domains $\left\{\bar{\Omega}_{k}\right\}, k=1,2, \ldots \mathcal{N}_{s}$. The regions of physical space occupied by the fluid and solid may evolve in time as the interfaces between the 
regions evolve in time. For each fluid domain $\Omega_{k}(t)$, which is assumed to contain an inviscid compressible fluid, we solve the Euler equations in terms of the physical space coordinate $\mathbf{x}$ and time $t$. These equations may be written in the conservation form

$$
\partial_{t} \mathbf{w}+\nabla_{\mathbf{x}} \cdot \mathbf{f}(\mathbf{w})=0, \quad \mathbf{x} \in \Omega_{k}(t), \quad t>0,
$$

where $\mathbf{w}=[\rho, \rho \mathbf{v}, \rho E]^{T}$ is the vector of conserved variables (mass, momentum, energy) and $\mathbf{f}=[\rho \mathbf{v}, \rho \mathbf{v} \otimes \mathbf{v}+$ $p \mathbf{I},(\rho E+p) \mathbf{v}]^{T}$ is the flux. The total energy is given by $\rho E=p /(\gamma-1)+\frac{1}{2} \rho|\mathbf{v}|^{2}$ assuming an ideal gas with a constant ratio of specific heats. Within each solid domain $\bar{\Omega}_{k}$ we solve the equations of linear elasticity which are written as a first-order system for displacement, velocity and stress in terms of the reference coordinate $\overline{\mathbf{x}}$ and time $t$. These equations are

$$
\partial_{t} \overline{\mathbf{u}}=\overline{\mathbf{v}}, \quad \bar{\rho} \partial_{t} \overline{\mathbf{v}}=\nabla_{\overline{\mathbf{x}}} \cdot \overline{\boldsymbol{\sigma}}, \quad \partial_{t} \overline{\boldsymbol{\sigma}}=S\left(\nabla_{\overline{\mathbf{x}}} \overline{\mathbf{v}}\right), \quad \overline{\mathbf{x}} \in \bar{\Omega}_{k}, \quad t>0,
$$

where $\overline{\boldsymbol{\sigma}}=\mathcal{S}\left(\nabla_{\overline{\mathbf{x}}} \overline{\mathbf{u}}\right)$ denotes the stress-strain relationship given by $\mathcal{S}=\lambda\left(\nabla_{\overline{\mathbf{x}}} \cdot \overline{\mathbf{u}}\right) \mathbf{I}+\mu\left(\nabla_{\overline{\mathbf{x}}} \overline{\mathbf{u}}+\nabla_{\overline{\mathbf{x}}} \overline{\mathbf{u}}^{T}\right)$. In this formulation, the equations governing the solid are solved in each (static) reference domain $\bar{\Omega}_{k}$, and the evolution of the region occupied by the solid in physical space, $\mathbf{x}(\overline{\mathbf{x}}, t)=\overline{\mathbf{x}}+\overline{\mathbf{u}}(\overline{\mathbf{x}}, t)$, is determined from the displacement, $\overline{\mathbf{u}}(\overline{\mathbf{x}}, t)$, whose solution is obtained from (2).

The initial state of the fluid in $\Omega_{k}$ is given by $\mathbf{w}(\mathbf{x}, 0)=\mathbf{w}_{0}(\mathbf{x})$, while initial conditions for the solid in $\bar{\Omega}_{k}$ are given by $\overline{\mathbf{u}}(\overline{\mathbf{x}}, 0)=\overline{\mathbf{u}}_{0}(\overline{\mathbf{x}}), \overline{\mathbf{v}}(\overline{\mathbf{x}}, 0)=\overline{\mathbf{v}}_{0}(\overline{\mathbf{x}})$ and $\overline{\boldsymbol{\sigma}}(\overline{\mathbf{x}}, 0)=\mathcal{S}\left(\nabla_{\overline{\mathbf{x}}} \overline{\mathbf{u}}_{0}(\overline{\mathbf{x}})\right)$. At an interface $\mathcal{I}$ between the fluid and solid, we impose the interface conditions

$$
\left\{\begin{array}{l}
\mathbf{n}(\mathbf{x}, t) \cdot \overline{\mathbf{v}}(\overline{\mathbf{x}}, t)=\mathbf{n}(\mathbf{x}, t) \cdot \mathbf{v}(\mathbf{x}, t), \\
\overline{\mathbf{n}}(\overline{\mathbf{x}}) \cdot \overline{\boldsymbol{\sigma}}(\overline{\mathbf{x}}, t)=-\left(p(\mathbf{x}, t)-p_{e}\right) \mathbf{n}(\mathbf{x}, t),
\end{array} \quad \text { for } \mathbf{x} \in \mathcal{I} \text { and } \overline{\mathbf{x}} \in \overline{\mathcal{I}},\right.
$$

where $p_{e}$ is a pressure offset, $\mathbf{n}(\mathbf{x}, t)$ is the normal to the fluid interface, and $\overline{\mathbf{n}}(\overline{\mathbf{x}})$ is the corresponding normal to the solid reference domain ${ }^{3}$. Here, $\overline{\mathcal{I}}$ denotes the position of the interface in the solid reference frame corresponding to the interface $\mathcal{I}$ in the physical domain. The first condition in (3) is a kinematic condition involving the normal component of velocity (the inviscid equations allow slip) and the second is a dynamic condition involving a balance of forces on the surface of the interface. Note that the normal to the fluid domain, $\mathbf{n}(\mathbf{x}, t)$, is generally different from the normal to the solid reference domain, $\overline{\mathbf{n}}(\overline{\mathbf{x}})$, although under the assumption of small strains that lead to the equations of linear elasticity, these two normals will be nearly the same. The choice of which normals to use in (3) is thus somewhat arbitrary and we have made the given choice for convenience of implementation. Boundary conditions are also required for the remaining boundaries of the fluid and solid domains, and these can take various forms as described for the sample problems considered later.

\section{One-dimensional elastic piston}

The one-dimensional elastic-piston problem is an instructive model problem for fluid structure interactions. Exact solutions of the problem can be obtained for various choices of the initial conditions, and these can be used to check the accuracy and stability of numerical schemes for FSI problems. In addition, we use the solution of the fluid-solid Riemann problem, a special case of the elastic-piston problem, to develop discrete approximations of the interface conditions (3) which have favorable stability and accuracy properties.

We consider a one-dimensional elastic-piston problem that couples the solution of a linear elastic solid (the "piston") and an inviscid compressible fluid. At $t=0$ the elastic solid occupies the domain $\bar{\Omega}=(-\infty, 0)$ and a compressible fluid occupies the adjacent domain $\Omega(0)=(0, \infty)$. (The subscripts on $\bar{\Omega}$ and $\Omega$ have been dropped for notational convenience.) As time evolves, the interface $\mathcal{I}$ between the solid and fluid traces the curve $x=G(t)$, while the corresponding position of the interface $\overline{\mathcal{I}}$ in the solid reference frame is given by $\bar{x}=0$, as shown in Figure 1. For this one-dimensional problem, the governing equations for the solid and fluid, given by (2) and (1), respectively, become

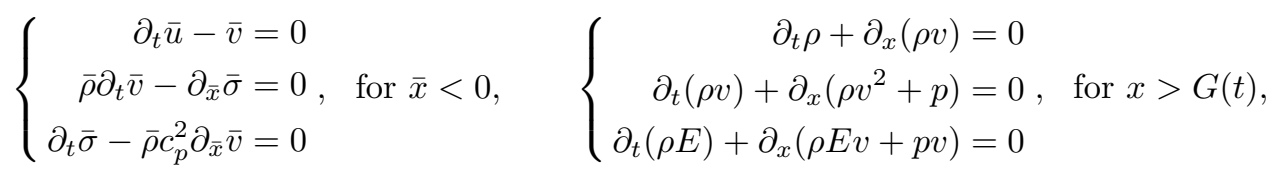

\footnotetext{
${ }^{3}$ We use the convention that at a fluid-solid interface both fluid and solid normals point into the fluid domain.
} 


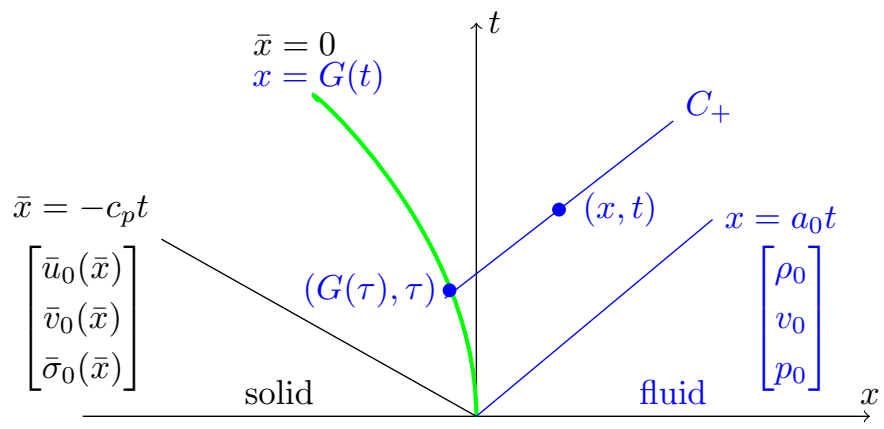

Figure 1: The $x$ - $t$ diagram for the elastic piston problem with a receding piston.

where $\rho E=p /(\gamma-1)+\rho v^{2} / 2$. As mentioned previously, the equations for the solid are solved in terms of a fixed reference coordinate $\bar{x}$ with the corresponding physical position given by $x=\bar{x}+\bar{u}$, where $\bar{u}$ is the computed displacement. In general, the initial conditions are taken to be

$$
\begin{aligned}
& {[\bar{u}(\bar{x}, 0), \bar{v}(\bar{x}, 0), \bar{\sigma}(\bar{x}, 0)]=\left[\bar{u}_{0}(\bar{x}), \bar{v}_{0}(\bar{x}), \bar{\rho} c_{p}^{2} \partial_{\bar{x}} \bar{u}_{0}(\bar{x})\right] \quad \text { for } \bar{x}<0,} \\
& {[\rho(x, 0), v(x, 0), p(x, 0)]=\left[\rho_{0}(x, 0), v_{0}(x, 0), p_{0}(x, 0)\right], \quad \text { for } x>0 \text {, }}
\end{aligned}
$$

and the interface conditions in (3) reduce to

$$
\left\{\begin{array}{l}
\bar{v}(\bar{x}, t)=v(x, t), \\
\bar{\sigma}(\bar{x}, t)=\sigma(x, t) \equiv-p(x, t)+p_{e}, \quad \text { for } x=G(t) \text { and } \bar{x}=0 .
\end{array}\right.
$$

We first consider the elastic-piston problem for the case where the initial states in (5) and (6) are taken to be constants. The solution of this fluid-solid Riemann problem is given in Section 4.1 for both the linearized and the full nonlinear problems. Solutions of these two fluid-solid Riemann problems are used to derive discrete approximations of the interface conditions which are then used in the FSI-DCG time-stepping algorithm. The details of this time-stepping algorithm in the context of the elastic-piston problem are given in Section 4.2. Finally, we consider numerical results for more general one-dimensional elastic-piston problems in order to demonstrate the stability and accuracy of the time-stepping scheme. These numerical results make use of two exact solutions, one involving a shock in the fluid hitting a solid piston at rest, and the other involving a gently receding piston. The first solution, given in Appendix $\mathrm{B}$, is used in part to test the robustness and accuracy of our scheme for non-smooth solutions and for the difficult case of a shock hitting a very light piston. The second solution, given in Appendix $\mathrm{A}$, is designed to test the convergence rate of the scheme in the maximum norm for a solution that is sufficiently smooth.

\subsection{The fluid-solid Riemann problem}

In this section we derive the solution to the fluid-solid Riemann (FSR) problem which will later be used to define a numerical approximation at the interface. The FSR problem is a special case of the elastic-piston problem where the initial conditions consist of a constant solid state next to a constant fluid state. The FSR problem is nonlinear in general, but we are also interested in a corresponding linearized FSR problem for the purpose of developing simpler discrete approximations.

At $t=0$ we assume that an elastic solid occupies the domain $\bar{x}<0$ with constant values $\left[\bar{v}_{0}, \bar{\sigma}_{0}\right]$ and a fluid occupies the domain $x>0$ with constant values $\left[\rho_{0}, v_{0}, p_{0}\right]$. Figure 2 shows the basic form of the solution in a space-time diagram. The fluid wave associated with the $C^{+}$characteristic field may be a shock or an expansion fan. The velocity and stress in the solid jump across the p-wave shock at $\bar{x}=-c_{p} t$. Let $\left[\rho^{*}, v^{*}, p^{*}\right]$ denote the fluid state adjacent to the interface and let $\left[\bar{v}^{*}, \bar{\sigma}^{*}\right]$ denote the solid state between the p-wave and the interface. The interface conditions imply $\bar{v}^{*}=v^{*}$ and $\bar{\sigma}^{*}=-p^{*}+p_{e}$, and the interface moves with the constant velocity so that its position is given by $x=G(t)=v^{*} t=\bar{v}^{*} t$.

\subsubsection{The linearized fluid-solid Riemann problem}

We first consider the solution of the fluid-solid Riemann problem in which the fluid equations in (4) are linearized about the constant initial state $\left[\rho_{0}, v_{0}, p_{0}\right]$. To better see the symmetry between the fluid and solid 


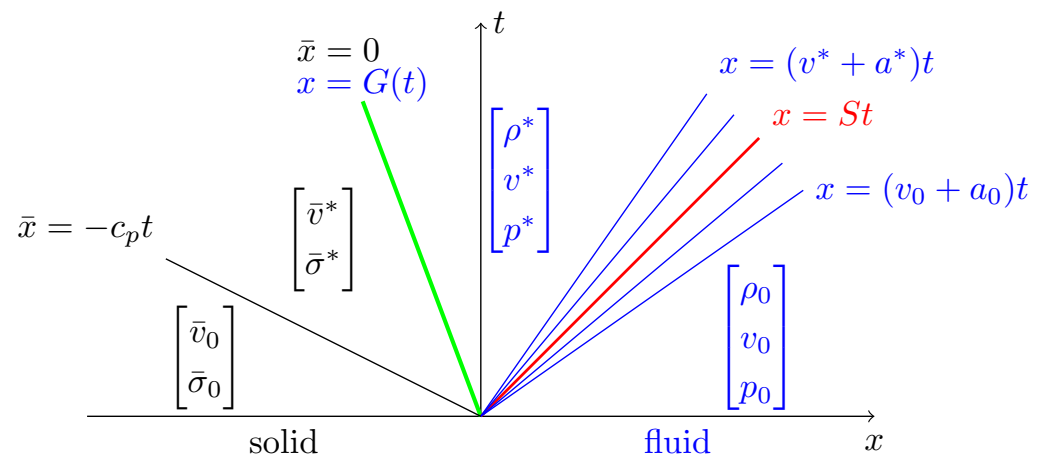

Figure 2: The $x$ - $t$ diagram for the fluid-solid Riemann problem. The wave on the right may be a shock with speed $S$ or an expansion fan. The interface between the fluid and solid is given by $\bar{x}=0$ in the solid and $x=v^{*} t=\bar{v}^{*} t$.

equations for this problem, we introduce a fluid stress $\sigma=-p+p_{e}$ and the corresponding constant states $\sigma_{0}=-p_{0}+p_{e}$ and $\sigma^{*}=-p^{*}+p_{e}$. The linearized equations are

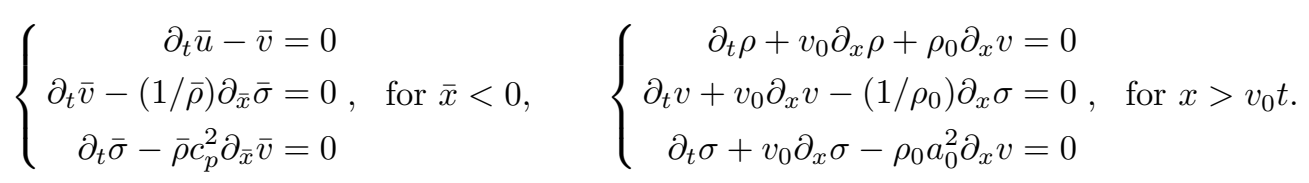

The characteristic relations for these hyperbolic equations are

$$
\left\{\begin{array} { r l r l } 
{ d \overline { u } / d t = \overline { v } , } & { } & { \text { on } d \overline { x } / d t = 0 , } \\
{ \overline { z } \overline { v } \mp \overline { \sigma } = \overline { z } \overline { v } _ { 0 } \mp \overline { \sigma } _ { 0 } , } & { } & { \text { on } d \overline { x } / d t = \pm c _ { p } , }
\end{array} \quad \left\{\begin{array}{rlrl}
a_{0}^{2} \rho+\sigma=a_{0}^{2} \rho_{0}+\sigma_{0}, & & \text { on } d x / d t=v_{0}, \\
z v \mp \sigma=z v_{0} \mp \sigma_{0}, & & \text { on } d x / d t=v_{0} \pm a_{0},
\end{array}\right.\right.
$$

where $\bar{z}=\bar{\rho} c_{p}$ and $z=\rho_{0} a_{0}$ are the acoustic impedances of the solid and fluid, respectively. Using the equations in (9) for the $C^{+}$characteristic in the solid and the $C^{-}$characteristic in the fluid, along with the interface conditions

$$
\left\{\begin{array}{l}
\bar{v}(\bar{x}, t)=v(x, t), \\
\bar{\sigma}(\bar{x}, t)=\sigma(x, t),
\end{array} \quad \text { for } x=v_{0} t \text { and } \bar{x}=0,\right.
$$

gives $\bar{z} v^{*}-\sigma^{*}=\bar{z} \bar{v}_{0}-\bar{\sigma}_{0}$ and $z v^{*}+\sigma^{*}=z v_{0}+\sigma_{0}$. Whence,

$$
\begin{aligned}
v^{*} & =\bar{v}^{*}=\frac{\bar{z} \bar{v}_{0}+z v_{0}}{\bar{z}+z}+\frac{\sigma_{0}-\bar{\sigma}_{0}}{\bar{z}+z}, \\
\sigma^{*} & =\bar{\sigma}^{*}=\frac{\bar{z}^{-1} \bar{\sigma}_{0}+z^{-1} \sigma_{0}}{\bar{z}^{-1}+z^{-1}}+\frac{v_{0}-\bar{v}_{0}}{\bar{z}^{-1}+z^{-1}} .
\end{aligned}
$$

The density of the fluid adjacent to the interface is given by $\rho^{*}=\rho_{0}-\left(\sigma^{*}-\sigma_{0}\right) / a_{0}^{2}$, which is obtained using the $C^{0}$ characteristic equation in (9) that holds along particle paths $d x / d t=v_{0}$ in the fluid. This condition is a linearized form of the entropy condition

$$
\rho^{*}=\rho_{0}\left(p^{*} / p_{0}\right)^{1 / \gamma}
$$

In practice, we have found this latter condition to be preferable to the lineared form for use in numerical simulations. Denote this solution, (11), (12) and (13) to the linearized FSR problem by

$$
\left[\rho^{*}, v^{*}, p^{*} ; \bar{v}^{*}, \bar{\sigma}^{*}\right]=\mathcal{L} \mathcal{F} \mathcal{S R}\left(\rho_{0}, v_{0}, p_{0} ; \bar{v}_{0}, \bar{\sigma}_{0}\right)
$$

The results in (11) and (12) show that the velocity and stress in the fluid (and in the solid) adjacent to the interface are given by impedance-weighted combinations of the initial states of the fluid and solid. In particular, we note that the commonly used velocity-from-solid/stress-from-fluid (VS/SF) interface approximation, $v_{I}=\bar{v}^{*}$ and $\sigma_{I}=\sigma^{*}$, corresponds to (11) and (12) in the limit of a heavy solid, i.e. $z / \bar{z} \rightarrow 0$, provided we also neglect the terms proportional to $\sigma_{0}-\bar{\sigma}_{0}$ and $v_{0}-\bar{v}_{0}$. (For smooth solutions these latter terms are approximations to the interface conditions themselves and so are small.) The results in (11), (12) and (13) are used later to derive discrete approximations for the states on either side of the fluid-solid interface. 


\subsubsection{The nonlinear fluid-solid Riemann problem}

The solution of the nonlinear FSR problem consists of two cases. In the first case there is a shock in the fluid in the $C^{+}$characteristic field, while in the second case there is an expansion fan (these two cases are illustrated in Figure 2). Consider first the case of a shock in the fluid traveling with speed $S$. The jump conditions at the shock imply

$$
\rho^{*}=\rho_{0} \frac{(\gamma+1) M^{2}}{(\gamma-1) M^{2}+2}, \quad p^{*}=p_{0}\left(1+\frac{2 \gamma}{\gamma+1}\left(M^{2}-1\right)\right), \quad v^{*}=v_{0}+a_{0} \frac{2\left(M^{2}-1\right)}{(\gamma+1) M},
$$

where $M=\left(S-v_{0}\right) / a_{0}$ is the Mach number of the shock relative to the flow ahead (see, e.g., Whitham [28]). Combining the jump conditions in (15), the $C^{+}$characteristic relation in the solid (i.e. $\bar{z} \bar{v}^{*}-\sigma^{*}=\bar{z} \bar{v}_{0}-\bar{\sigma}_{0}$ ), and the interface conditions gives the following cubic equation for $M$ :

$$
\left(1+\frac{2 \gamma}{\gamma+1}\left(M^{2}-1\right)+\frac{\bar{\sigma}_{0}-p_{e}}{p_{0}}\right) \frac{1}{\gamma} \frac{\rho_{0} a_{0}}{\bar{\rho} c_{p}}+\frac{v_{0}-\bar{v}_{0}}{a_{0}}=\frac{2\left(1-M^{2}\right)}{(\gamma+1) M} .
$$

After solving (16) for the physically relevant root $M$, the state of the fluid adjacent to the interface is given by (15) provided $M \geq 1$. The state of the solid adjacent to the interface is given by $\bar{v}^{*}=v^{*}$ and $\bar{\sigma}^{*}=-p^{*}+p_{e}$. If $M<1$, then an expansion fan in the $C^{+}$characteristic field is indicated. For this case, we use the characteristic equations

$$
\frac{2}{\gamma-1} a-v=\frac{2}{\gamma-1} a_{0}-v_{0}, \quad\left(\frac{a}{a_{0}}\right)^{2}=\left(\frac{\rho}{\rho_{0}}\right)^{\gamma-1}=\left(\frac{p}{p_{0}}\right)^{\frac{\gamma-1}{\gamma}},
$$

along with the interface conditions in (7) to obtain the following nonlinear equation satisfied by $p^{*}$,

$$
\frac{2}{\gamma-1} a_{0}\left(\frac{p^{*}}{p_{0}}\right)^{\frac{\gamma-1}{2 \gamma}}+\frac{p^{*}+\left(\bar{\sigma}_{0}-p_{e}\right)}{\bar{\rho} c_{p}}=\frac{2}{\gamma-1} a_{0}+\bar{v}_{0}-u_{0} .
$$

After solving this equation for $p^{*}$, the values of $a^{*}, \rho^{*}$, and $v^{*}$ are given using the characteristic equations in (17). The velocity in the expansion fan varies linearly with $x$ and is given by

$$
v(x, t)=v^{*}+\frac{x / t-\left(v^{*}+a^{*}\right)}{v_{0}+a_{0}-\left(v^{*}+a^{*}\right)}\left(v_{0}-v^{*}\right), \quad \text { for } v^{*}+a^{*}<x / t<v_{0}+a_{0} .
$$

Given $v(x, t)$ in the fan, $a(x, t), \rho(x, t)$ and $p(x, t)$ in the fan follow from (17).

In summary, the solution to the nonlinear fluid-solid Riemann problem breaks into two cases corresponding to the existence of a shock $(M \geq 1)$ or rarefaction $(M<1)$ in the fluid as indicated by the solution of (16). For the shock case with $M \geq 1$, the solution in the fluid adjacent to the interface is given by (15). For the rarefaction case with $M<1$, the solution is given by (17)-(19). In either case we obtain a formula for the state adjacent to the interface in terms of the initial states. We denote this solution by

$$
\left[\rho^{*}, v^{*}, p^{*} ; \bar{v}^{*}, \bar{\sigma}^{*}\right]=\mathcal{F} \mathcal{S R}\left(\rho_{0}, v_{0}, p_{0} ; \bar{v}_{0}, \bar{\sigma}_{0}\right) .
$$

It can be shown that the solution to the nonlinear FSR problem is close to that of the linearized FSR problem when the jump at the interface is small, $\bar{v}_{0} \approx v_{0}$ and $\bar{\sigma}_{0} \approx \sigma_{0}$.

\subsection{The FSI time-stepping algorithm and discrete interface approximations}

In this section, the FSI time-stepping algorithm and discrete approximations used at the fluid-solid interface are described. For clarity, the algorithm will be presented in detail for the case of the one-dimensional elastic piston. The one-dimensional case is simpler to describe than the general multi-dimensional case and it contains all of the salient features of the approach. An advantage of the numerical approach discussed here is that it is stable for both limiting cases of a "heavy" solid next to a "light" fluid and a "light" solid next to a "heavy" fluid. This is in contrast to the aforementioned standard VS/SF approach which is known to be unstable for the case of a "light" solid next to a "heavy" fluid. Finally, the discussion here focuses on the numerical treatment of the interface, while the numerical approximation of the governing equations is discussed briefly in Section 5.2 for multi-dimensional problems with additional details found in [29, 22, 19]. The interface approximation used here and described below is an extension of the method developed in [27]. In [27] the authors derived the impedance weighted averages (11) and (12) for a linear elastic solid coupled 
to an acoustic fluid. They proved the stability of a numerical scheme using these approximations. The proof did not include the second terms $\left(\sigma_{0}-\bar{\sigma}_{0}\right) /(\bar{z}+z)$ and $\left(v_{0}-\bar{v}_{0}\right) /\left(\bar{z}^{-1}+z^{-1}\right)$ in (11) and (12), although the proof can be extended to handle these additional terms. In the current paper we show the benefit (perhaps necessity) of either keeping these additional terms, or using the full nonlinear solution of the FSR problem when treating problems with strong shocks. The more accurate treatment for ghost point values given here also differs from that in [27].

In the fluid domain of the elastic piston, the governing equations in (4) are transformed to a moving coordinate system $r=x-G(t)$. The solid equations are solved in the fixed reference coordinates $\bar{x}$, $\infty<\bar{x}<0$. The governing equations for the solid and the fluid become

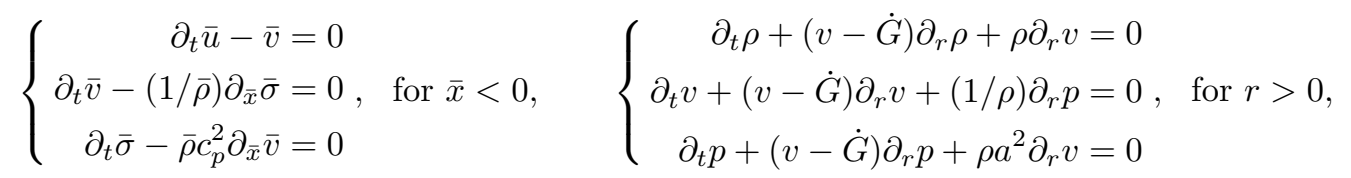

where $\rho=\rho(r, t), v=v(r, t)$ and $p=p(r, t)$ are now functions of $r$ and $t$, and $\dot{G}$ is the velocity of the interface. The interface jump conditions are

$$
\left\{\begin{array}{l}
\bar{v}(\bar{x}, t)=v(r, t) \\
\bar{\sigma}(\bar{x}, t)=-p(r, t)+p_{e}, \quad \text { for } r=0 \text { and } \bar{x}=0 .
\end{array}\right.
$$

The governing equations for the fluid are written here in non-conservative form since this clarifies the subsequent discussion of the numerical treatment of the interface. However, the numerical approximations of the fluid are developed for the equations in conservation form (see Section 5.2).

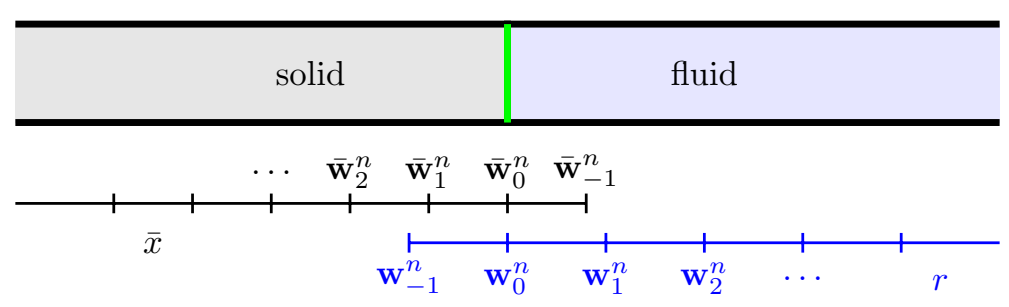

Figure 3: Grids for the elastic piston problem. The discrete solution for the solid is denoted $\overline{\mathbf{w}}_{i}^{n}$ and that for the fluid $\mathbf{w}_{i}^{n}$. Ghost point values $\overline{\mathbf{w}}_{-1}^{n}$ and $\mathbf{w}_{-1}^{n}$ are included.

To solve this elastic-piston problem numerically, we introduce a mesh for the solid domain $\bar{x}_{i}=-i \Delta \bar{x}$, $i=-1,0,1, \ldots$ and a mesh for the fluid domain $r_{i}=i \Delta r, i=-1,0,1, \ldots$, and introduce the corresponding discrete approximations $\mathbf{w}_{i}^{n}=\left[\rho_{i}^{n}, v_{i}^{n}, p_{i}^{n}\right]$ and $\overline{\mathbf{w}}_{i}^{n}=\left[\bar{u}_{i}^{n}, \bar{v}_{i}^{n}, \bar{\sigma}_{i}^{n}\right]$, where, for example, $\rho_{i}^{n} \approx \rho\left(r_{i}, n \Delta t\right)$, as shown in Figure 3. Suppose the discrete approximations $\overline{\mathbf{w}}_{i}^{n-1}$ and $\mathbf{w}_{i}^{n-1}, i=-1,0,1,2, \ldots$, are given at time $t^{n-1}=(n-1) \Delta t$. The algorithm for advancing the solution to the new time $t^{n}=n \Delta t$ consists of three stages. These stages are described in the subsections below and are summarized in Figure 4.

\subsubsection{Interior Stage}

The first stage of the time-stepping algorithm is a predictor step that uses approximations for the location of the fluid grid, $G^{p}$, and its velocity, $\dot{G}^{p}$, at time $t^{n}$. These two quantities are obtained by extrapolation in time from a time history of the grid locations, and are used in the numerical approximations of the PDEs in (21) to advance the solution to time $t^{n}$ at interior and interface points, i.e. we obtain $\overline{\mathbf{w}}_{i}^{n}$ and $\mathbf{w}_{i}^{n}$, for $i=0,1,2, \ldots$. Denote the predicted values for the fluid and solid on the interface by $\mathbf{w}_{0}^{n} \equiv\left[\rho_{0}, v_{0}, p_{0}\right]$ and $\overline{\mathbf{w}}_{0}^{n} \equiv\left[\bar{u}_{0}, \bar{v}_{0}, \bar{\sigma}_{0}\right]$, respectively, and let $\sigma_{0}=-p_{0}+p_{e}$. We note that these predicted values do not satisfy the interface conditions in general. For example, the interface velocity of the solid is generally different from that of the fluid, i.e. $\bar{v}_{0}^{n} \neq v_{0}^{n}$.

\subsubsection{Interface Stage}

The second stage of the time-stepping algorithm is a projection step that uses the predicted interface values $\overline{\mathbf{w}}_{0}^{n}$ and $\mathbf{w}_{0}^{n}$ to determine new interface values that satisfy the jump conditions in (22). These new interface values, which we denote by $\rho_{I}, v_{I}, \sigma_{I}$ and $p_{I} \equiv-\sigma_{I}+p_{e}$, are found from the solution of the fluid-solid Riemann problem as described in Section 4.1 with initial left and right states taken to be $\overline{\mathbf{w}}_{0}^{n}$ and $\mathbf{w}_{0}^{n}$, respectively. We solve the FSR problem in pseudo-time $t^{*}$ and determine the solution at the interface 


\begin{tabular}{|r|c|c|c|}
\hline \multicolumn{4}{|c|}{ The FSI time stepping algorithm } \\
\hline Stage & Condition & Type & Assigns \\
\hline Interior(a) & Predict grid and grid velocity & extrapolation & $G^{p}, \dot{G}^{p}$ \\
Interior(b) & Advance $\mathbf{w}_{i}^{n}, \overline{\mathbf{w}}_{i}^{n}, i=0,1,2, \ldots$ & PDE & interior, interface \\
Interface(a) & Compute $\left[v_{I}, \sigma_{I}, \rho_{I}\right]$ from (23) or (24) & projection & $v_{I}, \sigma_{I}, \rho_{I}$ \\
Interface(b) & Set $\rho_{0}^{n}=\rho_{I}, p_{0}^{n}=p_{I}, v_{0}=\bar{v}_{0}^{n}=v_{I}, \bar{\sigma}_{0}^{n}=\sigma_{I}$ & projection & $\mathbf{w}_{0}^{n}, \overline{\mathbf{w}}_{0}^{n}$ \\
Interface(c) & Correct $\bar{u}_{0}^{n}$, grid and grid velocity from (25) & projection & $\bar{u}_{0}^{n}, G^{n}, \dot{G}^{n}$ \\
Ghost(a) & $\mathbf{w}_{-1}^{n}=\mathcal{E}_{+1}^{(3)} \mathbf{w}_{0}^{n}, \overline{\mathbf{w}}_{-1}^{n}=\overline{\mathcal{E}}_{+1}^{(3)} \overline{\mathbf{w}}_{0}^{n}$, & extrapolation & $\mathbf{w}_{-1}^{n}, \overline{\mathbf{w}}_{-1}^{n}$ \\
Ghost(b) & Compute $\dot{\bar{v}}_{0}=(1 / \bar{\rho}) \bar{D}_{0} \bar{\sigma}_{0}^{n}, \ldots$ from (28) & PDE & $\dot{\bar{v}}_{0}, \dot{\bar{\sigma}}_{0}, \dot{v}_{0}, \dot{\sigma}_{0}$ \\
Ghost(c) & Compute $\dot{v}_{I}, \dot{\sigma}_{I}$ from (30) & projection & $\dot{v}_{I}, \dot{\sigma}_{I}$ \\
Ghost(d) & Set $(1 / \bar{\rho}) \bar{D}_{0} \bar{\sigma}_{0}^{n}=\dot{v}_{I}, \ldots$ from (31) & compatibility & $\bar{\sigma}_{-1}, \bar{v}_{-1}^{n}, p_{-1}^{n}, v_{-1}^{n}$ \\
\hline
\end{tabular}

Figure 4: The FSI time stepping algorithm and interface conditions for the case of a solid domain next to a fluid domain. Here $\mathbf{w}_{i}^{n}=\left[\rho_{i}^{n}, v_{i}^{n}, p_{i}^{n}\right]$ and $\overline{\mathbf{w}}_{i}^{n}=\left[\bar{u}_{i}^{n}, \bar{v}_{i}^{n}, \bar{\sigma}_{i}^{n}\right]$. The values on the interface are $\mathbf{w}_{0}^{n}, \overline{\mathbf{w}}_{0}^{n}$, while $\mathbf{w}_{-1}, \overline{\mathbf{w}}_{-1}$ denote the values at ghost points. The values of the solution are assigned in the order given.

for $t^{*}>0$. Using the solution to the linearized FSR problem $\mathcal{L F \mathcal { F }}\left(\rho_{0}, v_{0}, p_{0} ; \bar{v}_{0}, \bar{\sigma}_{0}\right)$ (equation 14), for example, gives the impedance weighted approximation

$$
\begin{aligned}
v_{I} & =\frac{\bar{z} \bar{v}_{0}+z v_{0}}{\bar{z}+z}+\frac{\sigma_{0}-\bar{\sigma}_{0}}{\bar{z}+z}, \\
\sigma_{I} & =\frac{\bar{z}^{-1} \bar{\sigma}_{0}+z^{-1} \sigma_{0}}{\bar{z}^{-1}+z^{-1}}+\frac{v_{0}-\bar{v}_{0}}{\bar{z}^{-1}+z^{-1}}, \\
\rho_{I} & =\rho_{0}\left(p_{I} / p_{0}\right)^{1 / \gamma} .
\end{aligned}
$$

Alternatively, we could use the solution of the nonlinear FSR problem, in which case

$$
\left[\rho_{I}, v_{I}, p_{I} ; \bar{v}_{I}, \bar{\sigma}_{I}\right]=\mathcal{F} \mathcal{S R}\left(\rho_{0}, v_{0}, p_{0} ; \bar{v}_{0}, \bar{\sigma}_{0}\right)
$$

where $\mathcal{F S R}$ is defined in Section 4.1.2. After obtaining the projected interface values, taken from (23) or (24), the predicted interface values are reassigned to be $\bar{v}_{0}^{n}=v_{I}, \bar{\sigma}_{0}^{n}=\sigma_{I}, \rho_{0}^{n}=\rho_{I}, v_{0}^{n}=v_{I}$ and $p_{0}^{n}=p_{I}$.

The predicted values for the solid displacement on the interface, $\bar{u}_{0}^{n}$, the grid location, $G^{p}$, and grid velocity $\dot{G}^{p}$ are also projected with an impedance weighted average to obtain the corrected values

$$
\bar{u}_{0}^{n}=u_{I} \equiv \frac{\bar{z} \bar{u}_{0}+z G^{p}}{\bar{z}+z}, \quad G^{n}=u_{I}, \quad \dot{G}^{n}=v_{I} .
$$

No further iterations are required to determine the interface and grid values.

For smooth solutions, the interface values in (23) are expected to provide a good approximation since the linearized solution of the FSR problem is a good approximation to the nonlinear solution. Also, the terms proportional to $\sigma_{0}-\bar{\sigma}_{0}$ and $v_{0}-\bar{v}_{0}$ in (23) can be neglected since these are of the order of the mesh spacing squared for smooth solutions. In this case the projection operators provide an impedance-weighted average of the predicted interface values. For heavy solids and light fluids, i.e. $\bar{z} \gg z$, this projection reduces to the commonly used FSI approach which uses the velocity from the solid and the stress from the fluid (VS/SF), $v_{I}=\bar{v}_{0}, p_{I}=p_{0}, \rho_{I}=\rho_{0}$. However, for light solids and heavy fluids, i.e. $\bar{z} \ll z$, the projection uses the stress-from-solid/velocity-from-fluid (SS/VF) condition.

For problems with shocks, the simpler impedance-weighted averages do not always provide stable approximations. The situation of a shock in a heavy fluid hitting the interface with a light solid is particularly difficult. In this case we find it necessary to use either the interface values in (24) given by the solution of the nonlinear FSR problem or the interface values in (23) given by the linearized FSR problem and including the terms proportional to $\sigma_{0}-\bar{\sigma}_{0}$ and $v_{0}-\bar{v}_{0}$. Although (24) generally gives slightly better results, we recommend using the full formulas in (23) as they are easier to implement and give nearly as good results.

\subsubsection{Ghost Stage}

The third stage of the algorithm assigns the grid values $\overline{\mathbf{w}}_{-1}^{n}$ and $\mathbf{w}_{-1}^{n}$ at ghost points. This may be done using extrapolation of ghost point values as discussed in [27]. An alternate approach, and the one used here, involves an extrapolation followed by an update of the ghost values using compatibility conditions at the 
interface derived from the governing equations and interface conditions. We have found this latter approach to be more accurate and stable (in the sense of pressure and density positivity).

The first step involves an extrapolation, which is based on the third-order formulas

$$
\overline{\mathbf{w}}_{-1}^{n}=\Delta_{+}^{3} \overline{\mathbf{w}}_{0}^{n}=3 \overline{\mathbf{w}}_{0}-3 \overline{\mathbf{w}}_{1}+\overline{\mathbf{w}}_{2}, \quad \mathbf{w}_{-1}=\Delta_{+}^{3} \mathbf{w}_{0}^{n}=3 \mathbf{w}_{0}-3 \mathbf{w}_{1}+\mathbf{w}_{2} .
$$

These extrapolations are sufficient to obtain second-order accuracy for smooth solutions. To handle the more difficult case when shocks may hit the interface, we use a limited extrapolation denoted by

$$
\overline{\mathbf{w}}_{-1}^{n}=\overline{\mathcal{E}}_{+1}^{(3)} \overline{\mathbf{w}}_{0}^{n}, \quad \mathbf{w}_{-1}=\mathcal{E}_{+1}^{(3)} \mathbf{w}_{0}^{n},
$$

and described below in Section 4.2.4. We now consider the equations for velocity and stress in (21) evaluated at the interface, i.e. at $\bar{x}=r=0$. These are

$$
\partial_{t} \bar{v}=\frac{1}{\bar{\rho}} \partial_{\bar{x}} \bar{\sigma}, \quad \partial_{t} \bar{\sigma}=\bar{\rho} c_{p}^{2} \partial_{\bar{x}} \bar{v}, \quad \partial_{t} v=-\frac{1}{\rho} \partial_{r} p, \quad \partial_{t} p=-\rho a^{2} \partial_{r} v
$$

A discretization of the equations in (27) that uses the extrapolated values at the ghost points gives approximations

$$
\dot{\bar{v}}_{0}=\frac{1}{\bar{\rho}} \bar{D}_{0} \bar{\sigma}_{0}^{n}, \quad \dot{\bar{\sigma}}_{0}=\bar{\rho} c_{p}^{2} \bar{D}_{0} \bar{v}_{0}^{n}, \quad \dot{v}_{0}=-\frac{1}{\rho_{0}^{n}} D_{0} p_{0}^{n}, \quad \dot{\sigma}_{0}=\rho_{0}^{n}\left(a_{0}^{n}\right)^{2} D_{0} v_{0}^{n},
$$

where $\bar{D}_{0} \bar{v}_{i}=\left(\bar{v}_{i+1}-\bar{v}_{i-1}\right) /(-2 \Delta \bar{x})$ and $D_{0} v_{i}=\left(v_{i+1}-v_{i-1}\right) /(2 \Delta r)$ are the centered divided difference operators, and $\dot{\bar{v}}_{0}, \dot{\bar{\sigma}}_{0}, \dot{v}_{0}$ and $\dot{\sigma}_{0}$ are second-order accurate approximations for $\partial_{t} \bar{v}, \partial_{t} \bar{\sigma}, \partial_{t} v$ and $-\partial_{t} p$, respectively, at $\bar{x}_{0}=r_{0}=0$. (There is a minus sign in the definition of $\bar{D}_{0} \bar{v}_{i}$ since $\bar{x}_{i+1}-\bar{x}_{i-1}=-2 \Delta \bar{x}_{\text {. }}$ ) We note that the centered difference approximations in (28) are equivalent to some one-sided difference approximations in view of the extrapolations in $(26)$.

The last step involves a consideration of the interface conditions in (22) for velocity and stress. These conditions can be differentiated in time to give

$$
\left\{\begin{array}{l}
\partial_{t} \bar{v}(\bar{x}, t)=\partial_{t} v(r, t), \\
\partial_{t} \bar{\sigma}(\bar{x}, t)=-\partial_{t} p(r, t), \quad \text { for } r=0 \text { and } \bar{x}=0
\end{array}\right.
$$

We enforce these interface conditions numerically using the projections in (11) and (12) based on the solution of linearized FSR problem and set

$$
\dot{v}_{I}=\frac{\bar{z} \dot{\bar{v}}_{0}+z \dot{v}_{0}}{\bar{z}+z}+\frac{\dot{\sigma}_{0}-\dot{\bar{\sigma}}_{0}}{\bar{z}+z}, \quad \dot{\sigma}_{I}=\frac{\bar{z}^{-1} \dot{\bar{\sigma}}_{0}+z^{-1} \dot{\sigma}_{0}}{\bar{z}^{-1}+z^{-1}}+\frac{\dot{v}_{0}-\dot{\bar{v}}_{0}}{\bar{z}^{-1}+z^{-1}} .
$$

We now use the computed values for $\dot{v}_{I}$ and $\dot{\sigma}_{I}$ defined in (30) in the approximations for the governing equations to give

$$
\frac{1}{\bar{\rho}} \bar{D}_{0} \bar{\sigma}_{0}^{n}=\dot{v}_{I}, \quad \bar{\rho} c_{p}^{2} \bar{D}_{0} \bar{v}_{0}^{n}=\dot{\sigma}_{I}, \quad-\frac{1}{\rho_{0}^{n}} D_{0} p_{0}^{n}=\dot{v}_{I}, \quad \rho_{0}^{n}\left(a_{0}^{n}\right)^{2} D_{0} v_{0}^{n}=\dot{\sigma}_{I} .
$$

The four equations in $(\underline{31})$ are solved to update the values of $\bar{\sigma}_{-1}, \bar{v}_{-1}^{n}, p_{-1}^{n}$ and $v_{-1}^{n}$ at ghost points. For example, $\bar{\sigma}_{-1}^{n}=\bar{\sigma}_{+1}^{n}+(2 \Delta \bar{x}) \bar{\rho} \dot{v}_{I}$.

\subsubsection{Limited extrapolation}

To obtain second-order accuracy on smooth solutions we extrapolate values at ghost points to third order, as described in Section 4.2.3. To prevent negative densities and pressures for problems with shocks it is necessary to apply a limiter to the extrapolated values for the fluid variables. In practice it is also necessary to impose a lower bound on the density $\left(\rho_{\min }\right)$ and pressure $\left(p_{\min }\right)$ to prevent these from becoming negative (for density and pressure fields that are order one in size, we typically use a lower bound of $\rho_{\min }=10^{-5}$ and $\left.p_{\min }=10^{-5}\right)$. The limited extrapolation procedure we use is similar to the approach described in [22] but different enough to warrant an explanation here. The limited extrapolation for the fluid pressure blends a third-order extrapolation and a first-order one, and is defined by

$$
\begin{aligned}
\delta_{1} & =p_{0}, \quad \delta_{2}=2 p_{0}-p_{1}, \quad \delta_{3}=3 p_{0}-3 p_{1}+p_{2}, \\
\alpha & =\min \left(1, \frac{c_{\delta}\left|\delta_{3}-\delta_{2}\right|}{\left|\delta_{3}\right|+\left|p_{0}\right|+\left|p_{1}\right|+\epsilon_{p}}\right), \\
p_{-1} & =(1-\alpha) \delta_{3}+\alpha \delta_{1},
\end{aligned}
$$


where $\epsilon_{p}$ is a small parameter that prevents division by zero and $c_{\delta}$ is taken as 2 . Note that the indicator function $\alpha$ should be $O\left(h^{2}\right)$ for smooth solutions and $O(1)$ in the presence of discontinuities. The fluid velocity uses the same limited extrapolation as the pressure. The limited extrapolation for the fluid density is similar to that for the pressure except that it blends a third-order extrapolation with an even symmetry condition and (34) takes the form

$$
\rho_{-1}= \begin{cases}\rho_{1} & \text { if } \delta_{2}<\rho_{\min } \text { or } \delta_{3}<\rho_{\min }, \\ (1-\alpha) \delta_{3}+\alpha \rho_{1} & \text { otherwise }\end{cases}
$$

where $\delta_{2}, \delta_{3}$ and $\alpha$ follow (32)-(33) but with the pressure replaced by the density. The solid variables use third-order extrapolation with no limiting.

\subsection{Numerical results for the one-dimensional elastic-piston}

In this section we present results of numerical simulations of elastic-piston problems in one space dimension. A code was implemented in MATLAB for this problem and used as a test bed to evaluate different interface discretization strategies. We find it useful to present these results here in order to more clearly point to the strengths and weaknesses of the various approaches before moving on to the more complex overlapping grid case in two dimensions. Note that these one-dimensional results were verified by the full two-dimensional code, and sample results are presented later in Section 6.1.

We solve the one-dimensional FSI problem for the elastic wave equation coupled to a compressible gas. The solid reference domain $[-1,0]$ is discretized with $N$ grid points. The fluid domain, which is initially located on $[0,1]$, is also discretized with $N$ grid points. We use a second-order accurate extension of Godunov's method that corresponds to the one-dimensional versions of the multi-dimensional schemes outlined in Section 5.2. The solution algorithm and treatment of the interface follows the time-stepping algorithm given in Figure 4.

\subsubsection{The smoothly receding piston}

As a first example, we consider the case of a smoothly receding elastic piston (see Figure 1) whose exact solution is given in Appendix A. The initial conditions in the fluid and solid are choosen so that the position of the interface moves according to a specified smooth function. We choose values $G_{a}=1$ and $q=4$ in equation (A.7) giving an interface motion of $G(t)=-t^{4} / 4$. The initial conditions for the fluid are constant and those for the solid are defined by (A.8). We take $\gamma=1.4, \rho_{0}=0.1, p_{0}=\rho_{0} / \gamma, \bar{\rho}=r_{\rho}, \lambda=r_{\rho} \lambda_{0}$, and $\mu=r_{\rho} \mu_{0}$. The parameter $r_{\rho}$ is varied to change the relative densities of the fluid and solid while at the same time keeping the speed of sound in the solid fixed, $c_{p}=\sqrt{\lambda_{0}+2 \mu_{0}}$. The ratio of acoustic impedance's is $z / \bar{z}=.1 /\left(r_{\rho} \sqrt{3}\right) \approx .06 / r_{\rho}$.

The numerical solution to the receding piston problem is computed on a sequence of grids of increasing resolution and the errors are computed from the known exact solution. The max-norm error of a fluid or solid component is defined to be the maximum of the absolute values of the errors for that component. The relative max-norm error is defined to be the max-norm error divided by the max-norm of the solution values.

Figures 5-7 present the max-norm relative-errors at $t=1.0$ for the three cases $r_{\rho}=10^{-5}, 1$ and $10^{5}$ corresponding to impedance ratios of $\bar{z} / z \approx 1.7 \times 10^{-4}, 1.7 \times 10^{1}$ and $1.7 \times 10^{6}$, respectively. These results were obtained using a time step equal to 0.8 times the maximum value allowed by either the fluid or solid solvers alone (i.e. we run at a CFL number equal to 0.8). Similiar errors and convergence rates are obtained for CFL numbers equal to 0.5 and 0.1. The values in the columns titled "r" give the ratio of the error at the current grid resolution to that of the next coarser grid resolution in the figure. The convergence rate is estimated from a least squares fit to the logarithm of the errors. These results we obtained using the linearized approximation (23) to the fluid-solid Riemann problem. Similar errors are obtained using the full nonlinear FSR solution. The results in the figures indicate that the solution remains stable and secondorder accurate over this wide range of impedance ratios. By comparison, numerical results for this problem obtained using the classical velocity-from-solid/stress-from-fluid (VS/SF) interface approximation indicate that the solution seems stable for approximately $\bar{z} / z \geq 1.5$ but becomes unstable for $\bar{z} / z \leq 1$.

\subsubsection{The elastic-piston shock tube}

As a second example we consider the solution to the elastic shock-tube problem given in Appendix B. A fluid shock moves from right to left and impacts the elastic solid causing a reflected wave (shock or rarefaction) in the fluid, and a transmitted p-wave in the solid. This case demonstrates that the interface approximations remain stable and accurate in the presence of shocks. The initial conditions, corresponding to a Mach 2 


\begin{tabular}{|l|c|c|c|c|c|c|c|c|c|c|c|c|c|}
\hline \multicolumn{1}{|c|}{} & \multicolumn{10}{c|}{ Fluid } \\
\hline grid & $\mathrm{N}$ & $\rho$ & $\mathrm{r}$ & $v$ & $\mathrm{r}$ & $p$ & $\mathrm{r}$ & $\bar{u}$ & $\mathrm{r}$ & $\bar{v}$ & $\mathrm{r}$ & $\bar{\sigma}$ & $\mathrm{r}$ \\
\hline$G_{1}$ & 20 & $2.7 \mathrm{e}-03$ & & $2.1 \mathrm{e}-03$ & & $1.3 \mathrm{e}-03$ & & $3.9 \mathrm{e}-04$ & & $1.2 \mathrm{e}-04$ & & $1.6 \mathrm{e}-04$ & \\
$G_{2}$ & 40 & $6.1 \mathrm{e}-04$ & 4.5 & $5.2 \mathrm{e}-04$ & 4.1 & $3.1 \mathrm{e}-04$ & 4.2 & $9.7 \mathrm{e}-05$ & 4.1 & $3.4 \mathrm{e}-05$ & 3.6 & $3.2 \mathrm{e}-05$ & 5.2 \\
$G_{3}$ & 80 & $1.4 \mathrm{e}-04$ & 4.2 & $1.1 \mathrm{e}-04$ & 4.5 & $7.9 \mathrm{e}-05$ & 3.9 & $2.4 \mathrm{e}-05$ & 4.1 & $8.5 \mathrm{e}-06$ & 4.0 & $6.9 \mathrm{e}-06$ & 4.6 \\
$G_{4}$ & 160 & $3.5 \mathrm{e}-05$ & 4.2 & $3.0 \mathrm{e}-05$ & 3.9 & $2.0 \mathrm{e}-05$ & 4.0 & $5.9 \mathrm{e}-06$ & 4.0 & $2.1 \mathrm{e}-06$ & 4.0 & $1.6 \mathrm{e}-06$ & 4.3 \\
\hline rate & & 2.10 & & 2.06 & & 2.01 & & 2.02 & & 1.95 & & 2.23 & \\
\hline
\end{tabular}

Figure 5: Max-norm errors at $t=1.0$ for the smoothly receding elastic-piston, very light solid case, $r_{\rho}=10^{-5}$, solid to fluid impedance ratio $\bar{z} / z=1.7 \times 10^{-4}$.

\begin{tabular}{|l|c|c|c|c|c|c|c|c|c|c|c|c|c|}
\hline \multicolumn{9}{|c|}{} & \multicolumn{9}{|c|}{ Fluid } & \multicolumn{7}{c|}{ Solid } \\
\hline grid & $\mathrm{N}$ & $\rho$ & $\mathrm{r}$ & $v$ & $\mathrm{r}$ & $p$ & $\mathrm{r}$ & $\bar{u}$ & $\mathrm{r}$ & $\bar{v}$ & $\mathrm{r}$ & $\bar{\sigma}$ & $\mathrm{r}$ \\
\hline$G_{1}$ & 20 & $1.6 \mathrm{e}-03$ & & $1.3 \mathrm{e}-03$ & & $1.1 \mathrm{e}-03$ & & $2.4 \mathrm{e}-04$ & & $1.2 \mathrm{e}-05$ & & $1.6 \mathrm{e}-05$ & \\
$G_{2}$ & 40 & $3.2 \mathrm{e}-04$ & 4.9 & $2.8 \mathrm{e}-04$ & 4.7 & $2.2 \mathrm{e}-04$ & 5.2 & $5.8 \mathrm{e}-05$ & 4.0 & $3.0 \mathrm{e}-06$ & 4.0 & $3.9 \mathrm{e}-06$ & 4.1 \\
$G_{3}$ & 80 & $6.9 \mathrm{e}-05$ & 4.7 & $4.9 \mathrm{e}-05$ & 5.7 & $4.5 \mathrm{e}-05$ & 4.8 & $1.4 \mathrm{e}-05$ & 4.1 & $7.5 \mathrm{e}-07$ & 4.0 & $9.1 \mathrm{e}-07$ & 4.3 \\
$G_{4}$ & 160 & $1.7 \mathrm{e}-05$ & 4.0 & $1.2 \mathrm{e}-05$ & 4.1 & $1.1 \mathrm{e}-05$ & 4.1 & $3.6 \mathrm{e}-06$ & 4.0 & $1.9 \mathrm{e}-07$ & 3.9 & $2.2 \mathrm{e}-07$ & 4.1 \\
\hline rate & & 2.17 & & 2.28 & & 2.23 & & 2.02 & & 1.99 & & 2.07 & \\
\hline
\end{tabular}

Figure 6: Max-norm relative errors at $t=1.0$ for the smoothly receding elastic-piston, medium solid case, $r_{\rho}=1$, solid to fluid impedance ratio $\bar{z} / z=1.7 \times 10^{1}$.

\begin{tabular}{|l|c|c|c|c|c|c|c|c|c|c|c|c|c|}
\hline \multicolumn{9}{|c|}{} & \multicolumn{9}{|c|}{ Fluid } & \multicolumn{7}{c|}{ Solid } \\
\hline grid & $\mathrm{N}$ & $\rho$ & $\mathrm{r}$ & $v$ & $\mathrm{r}$ & $p$ & $\mathrm{r}$ & $\bar{u}$ & $\mathrm{r}$ & $\bar{v}$ & $\mathrm{r}$ & $\bar{\sigma}$ & $\mathrm{r}$ \\
\hline$G_{1}$ & 20 & $1.6 \mathrm{e}-03$ & & $1.3 \mathrm{e}-03$ & & $1.1 \mathrm{e}-03$ & & $2.4 \mathrm{e}-04$ & & $1.7 \mathrm{e}-05$ & & $1.6 \mathrm{e}-05$ & \\
$G_{2}$ & 40 & $3.2 \mathrm{e}-04$ & 4.9 & $2.8 \mathrm{e}-04$ & 4.8 & $2.2 \mathrm{e}-04$ & 5.2 & $5.9 \mathrm{e}-05$ & 4.0 & $4.0 \mathrm{e}-06$ & 4.3 & $3.7 \mathrm{e}-06$ & 4.3 \\
$G_{3}$ & 80 & $6.7 \mathrm{e}-05$ & 4.8 & $5.0 \mathrm{e}-05$ & 5.6 & $4.6 \mathrm{e}-05$ & 4.7 & $1.4 \mathrm{e}-05$ & 4.1 & $9.4 \mathrm{e}-07$ & 4.2 & $9.0 \mathrm{e}-07$ & 4.1 \\
$G_{4}$ & 160 & $1.7 \mathrm{e}-05$ & 4.0 & $1.2 \mathrm{e}-05$ & 4.1 & $1.1 \mathrm{e}-05$ & 4.0 & $3.6 \mathrm{e}-06$ & 4.0 & $2.3 \mathrm{e}-07$ & 4.1 & $2.3 \mathrm{e}-07$ & 3.9 \\
\hline rate & & 2.19 & & 2.28 & & 2.22 & & 2.02 & & 2.07 & & 2.03 & \\
\hline
\end{tabular}

Figure 7: Max-norm relative errors at $t=1.0$ for the smoothly receding elastic-piston, very heavy solid case, $r_{\rho}=10^{5}$, solid to fluid impedance ratio $z / \bar{z}=1.7 \times 10^{6}$.

shock in the fluid moving with speed $S_{0}=-2$ are $\left[\rho_{0}, v_{0}, p_{0}\right]=[1,0,1 / \gamma],\left[\rho_{1}, v_{1}, p_{1}\right]=[8 / 3,-5 / 4,45 / 14]$ with $\gamma=1.4$ and $\left[\bar{v}_{0}, \bar{\sigma}_{0}\right]=[0,0]$ with $\bar{\rho}=r_{\rho}, \lambda=r_{\rho} \lambda_{0}$, and $\mu=r_{\rho} \mu_{0}$. The shock is initially located at $x_{0}=.25$ and the pressure offset is chosen as $p_{e}=p_{0}$ so that the solid is initially at rest with respect to the fluid state $\left[\rho_{0}, v_{0}, p_{0}\right]$.

Figures $8-10$ show the computed solutions in the solid and fluid domains for the three cases $r_{\rho}=.01,1$ and 50. For the solid domain the quantities $\bar{u}, \bar{v}$, and $\bar{\sigma} / \bar{z}$ are plotted. Note that $\bar{v}$ and $\bar{\sigma} / \bar{z}$ lie directly atop each other in the plots. For the fluid domain the quantities $\rho,-v$ and $p$ are shown. By the times shown the incident shock has interacted with the interface to produce a transmitted traveling discontinuity in the solid and a reflected wave in the fluid. For the case of a heavy solid, the reflected wave in the fluid is a shock while for the light solid case it is an expansion fan. The blip in the fluid density at the piston face, especially noticeable in Figure 10, is a result of the usual wall heating phenomena [30].

Figures $11-13$ give the $L_{1}$-norm relative errors for the three cases $r_{\rho}=10^{5}, 1,10^{-5}$ corresponding to $z / \bar{z} \approx 6 \times 10^{4}, 0.6$, and $6 \times 10^{-6}$. The expected $L_{1}$ convergence rate for solutions with linear discontinuities (such as the discontinuous p-wave in the solid solution) is $2 / 3$ [31], and for non-linear discontinuities (such as the shock in the fluid) is 1 . The results in the figures are consistent with these expected rates.

The case of a gas shock hitting a light solid is particularly interesting and challenging. For this case, we found that it is important to use the full solution of the linearized FSR problem (23) or the solution of the nonlinear fluid-solid Riemann problem (24) to obtain stable and accurate solutions. In particular, it was found that the use of the simplified form of the solution of the linear FSR problem (i.e. without the terms proportional to $\sigma_{0}-\bar{\sigma}_{0}$ and $v_{0}-\bar{v}_{0}$ ) results in a less robust scheme which generally gives less accurate solutions. 

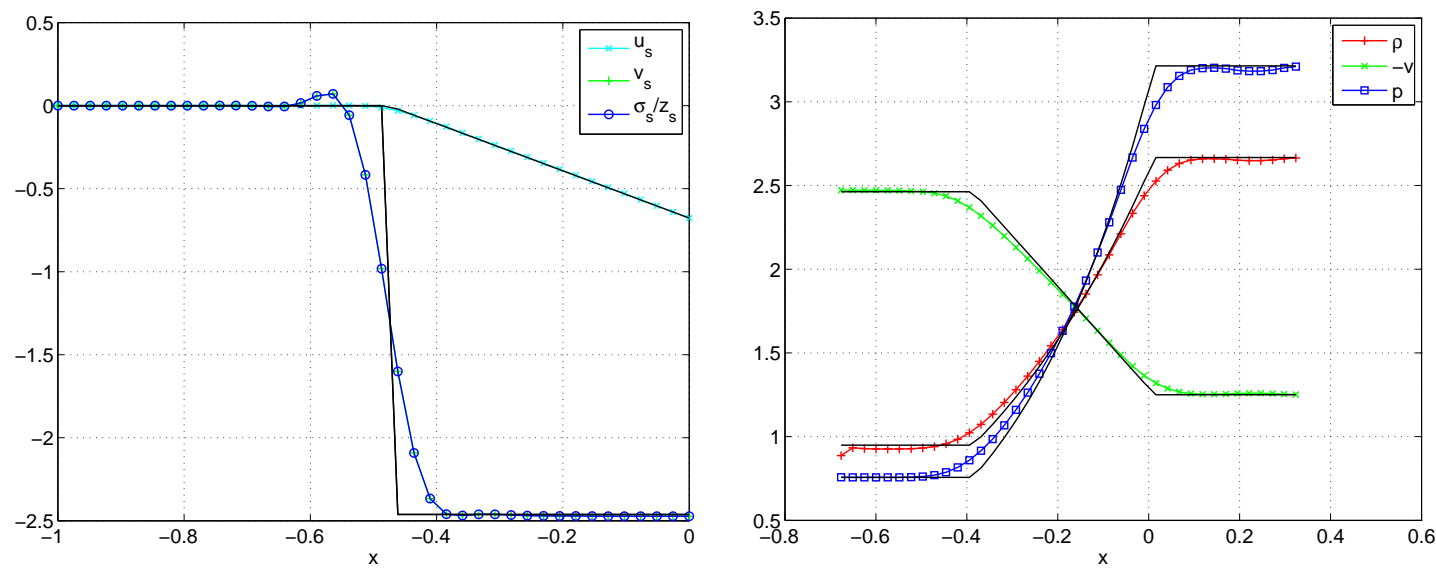

Figure 8: Elastic-piston shock tube results at $t=0.4$ for the light solid, $r_{\rho}=.01$. Left: solid. Right fluid. The interface approximation uses the solution to the linearized FSR problem. The solid black lines indicate the exact solution.
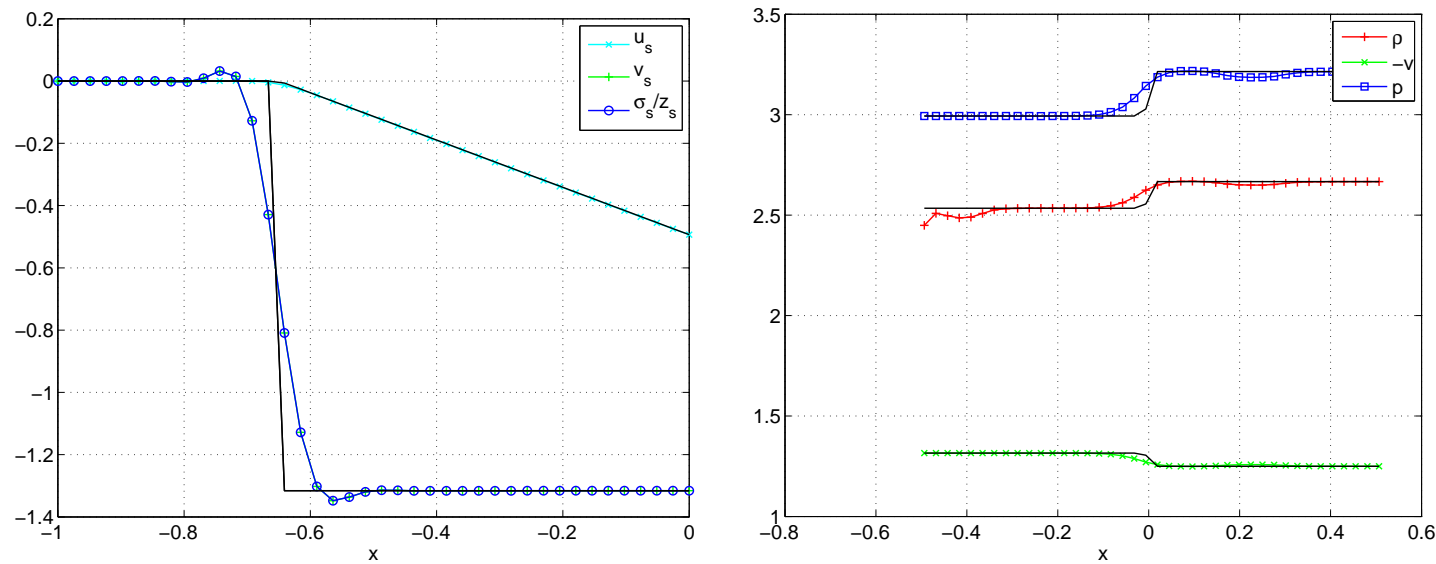

Figure 9: Elastic shock tube results at $t=0.5$ for the medium solid, $r_{\rho}=1$. Left: solid. Right fluid. The interface approximation uses the solution to the linearized FSR problem. The solid black lines indicate the exact solution.
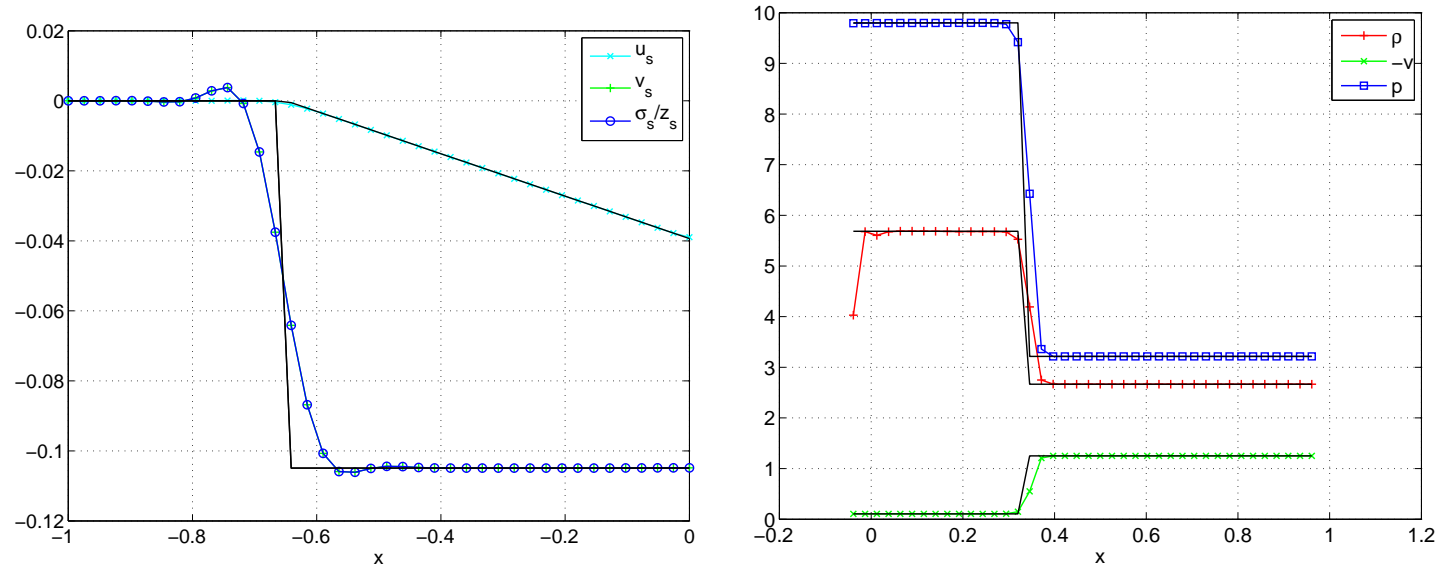

Figure 10: Elastic-piston shock tube results at $t=0.5$ for the heavy solid, $r_{\rho}=50$.. Left: solid. Right fluid. The interface approximation uses the solution to the linearized FSR problem. The solid black lines indicate the exact solution.

\section{The FSI-DCG numerical approach for initial-boundary-value problems}

Our numerical approach for the solution of the equations governing an FSI initial-boundary-value problem is based on the use of composite overlapping grids with locally deforming grids adjacent to the interfaces. Section 5.1 begins with a brief overview of overlapping grids and then proceeds to a discussion of the deforming composite grid approach. A more detailed discussion of the overlapping grid approach may be found in $[26,32,33]$, for example. The discretization of the fluid and solid equations on their respective domains is briefly described in Section 5.2 . The extension of the one-dimensional FSI time-stepping algorithm introduced in Section 4.2 to multiple space dimensions is discussed in Section 5.3 . 


\begin{tabular}{|l|c|c|c|c|c|c|c|c|c|c|c|c|c|}
\hline \multicolumn{1}{|c|}{} & \multicolumn{10}{c|}{ Fluid } \\
\hline grid & $\mathrm{N}$ & $\rho$ & $\mathrm{r}$ & $v$ & $\mathrm{r}$ & $p$ & $\mathrm{r}$ & $\bar{u}$ & $\mathrm{r}$ & $\bar{v}$ & $\mathrm{r}$ & $\bar{\sigma}$ & $\mathrm{r}$ \\
\hline$G_{1}$ & 20 & $2.2 \mathrm{e}-02$ & & $1.7 \mathrm{e}-02$ & & $2.3 \mathrm{e}-02$ & & $9.5 \mathrm{e}-03$ & & $6.2 \mathrm{e}-02$ & & $3.8 \mathrm{e}-06$ & \\
$G_{2}$ & 40 & $1.2 \mathrm{e}-02$ & 1.9 & $8.7 \mathrm{e}-03$ & 1.9 & $1.1 \mathrm{e}-02$ & 2.0 & $5.5 \mathrm{e}-03$ & 1.7 & $3.5 \mathrm{e}-02$ & 1.8 & $2.2 \mathrm{e}-06$ & 1.8 \\
$G_{3}$ & 80 & $6.6 \mathrm{e}-03$ & 1.7 & $4.3 \mathrm{e}-03$ & 2.0 & $5.7 \mathrm{e}-03$ & 2.0 & $2.3 \mathrm{e}-03$ & 2.4 & $2.0 \mathrm{e}-02$ & 1.8 & $1.2 \mathrm{e}-06$ & 1.8 \\
$G_{4}$ & 160 & $3.3 \mathrm{e}-03$ & 2.0 & $2.4 \mathrm{e}-03$ & 1.8 & $2.9 \mathrm{e}-03$ & 1.9 & $1.4 \mathrm{e}-03$ & 1.7 & $1.2 \mathrm{e}-02$ & 1.7 & $7.2 \mathrm{e}-07$ & 1.7 \\
\hline rate & & 0.91 & & 0.95 & & 0.99 & & 0.96 & & 0.80 & & 0.80 & \\
\hline
\end{tabular}

Figure 11: Elastic-piston shock tube convergence results for a very light solid, $r_{\rho}=10^{-5} ; L_{1}$-norm relative errors and estimated convergence rates at $t=0.5$.

\begin{tabular}{|l|c|c|c|c|c|c|c|c|c|c|c|c|c|}
\hline \multicolumn{9}{|c|}{} & \multicolumn{9}{|c|}{ Fluid } & \multicolumn{9}{c|}{ Solid } \\
\hline grid & $\mathrm{N}$ & $\rho$ & $\mathrm{r}$ & $v$ & $\mathrm{r}$ & $p$ & $\mathrm{r}$ & $\bar{u}$ & $\mathrm{r}$ & $\bar{v}$ & $\mathrm{r}$ & $\bar{\sigma}$ & $\mathrm{r}$ \\
\hline$G_{1}$ & 20 & $1.1 \mathrm{e}-02$ & & $4.7 \mathrm{e}-03$ & & $6.5 \mathrm{e}-03$ & & $4.6 \mathrm{e}-03$ & & $4.8 \mathrm{e}-02$ & & $3.6 \mathrm{e}-02$ & \\
$G_{2}$ & 40 & $5.4 \mathrm{e}-03$ & 2.0 & $2.8 \mathrm{e}-03$ & 1.7 & $3.8 \mathrm{e}-03$ & 1.7 & $2.0 \mathrm{e}-03$ & 2.4 & $2.7 \mathrm{e}-02$ & 1.8 & $2.1 \mathrm{e}-02$ & 1.8 \\
$G_{3}$ & 80 & $2.7 \mathrm{e}-03$ & 2.0 & $1.5 \mathrm{e}-03$ & 1.9 & $2.0 \mathrm{e}-03$ & 1.9 & $8.3 \mathrm{e}-04$ & 2.4 & $1.6 \mathrm{e}-02$ & 1.7 & $1.2 \mathrm{e}-02$ & 1.7 \\
$G_{4}$ & 160 & $1.5 \mathrm{e}-03$ & 1.8 & $8.2 \mathrm{e}-04$ & 1.8 & $1.1 \mathrm{e}-03$ & 1.8 & $4.0 \mathrm{e}-04$ & 2.1 & $9.5 \mathrm{e}-03$ & 1.7 & $7.2 \mathrm{e}-03$ & 1.7 \\
\hline rate & & 0.97 & & 0.85 & & 0.85 & & 1.19 & & 0.77 & & 0.77 & \\
\hline
\end{tabular}

Figure 12: Elastic-piston shock tube convergence results for a medium light solid, $r_{\rho}=1 ; L_{1}$-norm relative errors and estimated convergence rates at $t=0.5$.

\begin{tabular}{|l|c|c|c|c|c|c|c|c|c|c|c|c|c|}
\hline \multicolumn{1}{|c|}{} & \multicolumn{1}{|c|}{ Fluid } & \multicolumn{1}{c|}{ Solid } \\
\hline grid & $\mathrm{N}$ & $\rho$ & $\mathrm{r}$ & $v$ & $\mathrm{r}$ & $p$ & $\mathrm{r}$ & $\bar{u}$ & $\mathrm{r}$ & $\bar{v}$ & $\mathrm{r}$ & $\bar{\sigma}$ & $\mathrm{r}$ \\
\hline$G_{1}$ & 20 & $2.7 \mathrm{e}-02$ & & $1.8 \mathrm{e}-02$ & & $1.5 \mathrm{e}-02$ & & $5.2 \mathrm{e}-03$ & & $5.6 \mathrm{e}-02$ & & $5.3 \mathrm{e}-02$ & \\
$G_{2}$ & 40 & $1.4 \mathrm{e}-02$ & 1.9 & $1.4 \mathrm{e}-02$ & 1.3 & $7.8 \mathrm{e}-03$ & 1.9 & $1.8 \mathrm{e}-03$ & 2.8 & $3.2 \mathrm{e}-02$ & 1.8 & $3.0 \mathrm{e}-02$ & 1.8 \\
$G_{3}$ & 80 & $7.4 \mathrm{e}-03$ & 1.9 & $6.7 \mathrm{e}-03$ & 2.0 & $3.8 \mathrm{e}-03$ & 2.0 & $7.2 \mathrm{e}-04$ & 2.6 & $1.9 \mathrm{e}-02$ & 1.7 & $1.8 \mathrm{e}-02$ & 1.7 \\
$G_{4}$ & 160 & $3.8 \mathrm{e}-03$ & 2.0 & $3.4 \mathrm{e}-03$ & 2.0 & $1.9 \mathrm{e}-03$ & 2.0 & $2.7 \mathrm{e}-04$ & 2.6 & $1.1 \mathrm{e}-02$ & 1.7 & $1.1 \mathrm{e}-02$ & 1.7 \\
\hline rate & & 0.95 & & 0.82 & & 0.98 & & 1.41 & & 0.77 & & 0.77 & \\
\hline
\end{tabular}

Figure 13: Elastic-piston shock tube convergence results for a very heavy solid, $r_{\rho}=10^{5} ; L_{1}$-norm relative errors and estimated convergence rates at $t=0.5$.

\subsection{Deforming composite grids}

In the deforming-composite grid (DCG) approach for multi-domain problems, each fluid or solid domain is independently discretized with an overlapping grid. An overlapping grid, $\mathcal{G}$, consists of a set of structured component grids, $\left\{G_{g}\right\}, g=1, \ldots, \mathcal{N}$, that cover a domain, either $\Omega_{k}$ or $\bar{\Omega}_{k}$, and overlap where the component grids meet. Typically, boundary-fitted curvilinear grids are used near the boundaries while one or more background Cartesian grids are used to handle the bulk of the domain. Each component grid is a logically rectangular, curvilinear grid in $n_{d}$ space dimensions, and is defined by a smooth mapping from parameter space $\mathbf{r}$ (the unit square or cube) to physical space $\mathbf{x}$,

$$
\mathbf{x}=\mathbf{G}_{g}(\mathbf{r}), \quad \mathbf{r} \in[0,1]^{n_{d}}, \quad \mathbf{x} \in \mathbb{R}^{n_{d}} .
$$

The grid points on a component grid are marked as discretization points (where the governing equations or boundary conditions are discretized), unused points (points that are not used in the discretization) and interpolation points. Solution values at interpolation points are generally determined from a tensor-product Lagrange interpolant in the parameter space of the donor grid. Ghost points are used to facilitate the discretization of boundary conditions. The classification of grid points into discretization, interpolation and unused points (grid connectivity) is determined by an overlapping grid generator. We use the Ogen grid generator [34]. Ogen takes as input a set of overlapping component grids (for one or more domains) along with a classification of the boundaries of each grid as a physical boundary (including the interface between fluid and solid domains), an interpolation boundary or a periodic boundary. Unused points are determined by Ogen using physical boundaries to mark points exterior to the domain following a hole-cutting algorithm. The remaining points are classified as either discretization points or interpolation points.

In the FSI-DCG approach, component grids next to an interface deform over time to match the interface motion. This is illustrated in Figure 14. After the points on the interfaces have been evolved from one 

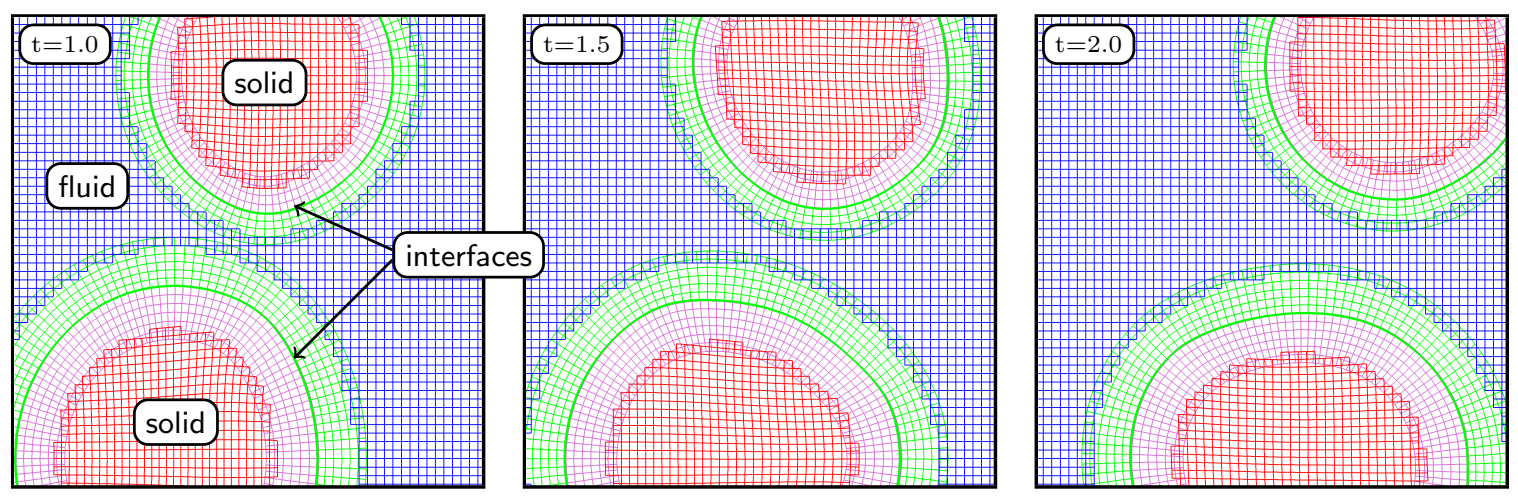

Figure 14: Composite grids at different times from the FSI-DCG simulation a shock hitting two solid elastic cylinders. The green fluid grids deform over time to follow the fluid-solid interfaces. The blue background Cartesian grid for the fluid remains fixed. The grids in the solid are shown adjusted for the displacements.

time step to the next, a hyperbolic grid generator is used to regenerate the interface grids. The hyperbolic grid generator starts from the interface curve (in two dimensions) or interface surface (in three dimensions), and marches the grid a specified distance into the domain by solving a hyperbolic-like PDE. Hyperbolic grid generators are a fast and commonly used technique for generating component grids for overlapping grids [35, 36, 37]. We use the hyperbolic grid generator from Overture which uses an implicit marching algorithm to construct the grid. The grids are constructed so as to remain smooth and nearly orthogonal. (See [38] for further details.)

Our PDE solution algorithms for deforming grids require the grid velocity to be given at each grid point. In addition, the acceleration of the grid is needed on boundaries to impose compatibility conditions there (e.g. estimating the normal derivative $\partial_{n} p$ at an interface from the momentum equation, see equation (42) below). The grid velocity and acceleration are determined on deforming grids from a time sequence of these grids using finite differences in time. The number of grid time levels we save is based on the order of accuracy of the time discretization. For the computations presented in this paper, we compute the grid velocity and acceleration to second-order using the latest 4 time levels. We note that the additional storage needed for the time sequence of grids is not large because this is done only for the narrow deforming grids attached to the interface.

\subsection{Discretization of the fluid and solid governing equations}

The numerical approximation of the Euler equations (1) follows the approach described in [29] for static grids and in [22] for moving rigid-body grids. The approximation of the equations of linear elasticity (2) for overlapping grids is described in [19]. Since these approximations have been discussed in detail elsewhere, we provide only a brief discussion of the discretizations here.

The first step in the numerical approach involves an exact mapping of the equations from physical space to computational space. For the Euler equations, consider a mapping for a (possibly moving) component grid given by $\mathbf{x}=\mathbf{G}(\mathbf{r}, t)$. Following the work in [22], the mapped equations are

$$
\partial_{t} \mathbf{w}+\frac{1}{J} \nabla_{\mathbf{r}} \cdot \mathbf{F}+\frac{\mathbf{w}}{J} \nabla_{\mathbf{r}} \cdot \mathbf{V}=0
$$

where $J=\left|\mathbf{x}_{\mathbf{r}}\right|$ is the Jacobian of the mapping, $\mathbf{F}$ is the mapped flux tensor, and $\mathbf{V}$ is related to the grid velocity $\dot{\mathbf{G}}$. In particular, the vector components of $\mathbf{F}$ and the scalar components of $\mathbf{V}$ are given by

$$
\mathbf{F}_{i}=\left(J \sum_{j=1}^{n_{d}} \frac{\partial r_{i}}{\partial x_{j}} \mathbf{f}_{j}\right)-V_{i} \mathbf{w}, \quad V_{i}=J \sum_{j=1}^{n_{d}} \frac{\partial r_{i}}{\partial x_{j}} \dot{\mathbf{G}}_{j}, \quad i=1, \ldots, n_{d} .
$$

The term in (35) involving the mapped flux with components $\mathbf{F}_{i}$ describes the flow of the conserved quantity $\mathbf{w}$ across fixed curves $r_{i}=$ constant, while the dilatation term involving the velocity $\mathbf{V}$ describes the change of $\mathbf{w}$ due to the compression or expansion of the grid. As noted in [22], the dilatation term is identically zero for the rigid-body motions (translations and rotations) considered in that work. For the current work, the fluid grids attached to interfaces deform as they evolve in time and thus the dilatation term is not zero in general. 
The numerical approximation of the mapped equation (35) employs a second-order extension of Godunov's method. A Roe-type approximate Riemann solver is used (other Riemann solvers are also available) to compute the mapped fluxes. Slope-limited updates of the left and right input states to the Riemann solver are used to obtain second-order accuracy (for smooth regions of flow). Centered differences are used to approximate the dilatation term. The scheme is adjusted to ensure free-stream preservation, including the case of deforming grids. Further details may be found in [29] and [22].

For the solid, we consider the governing equations in (2) written in the form

$$
\partial_{t} \overline{\mathbf{w}}+\nabla_{\overline{\mathbf{x}}} \cdot \overline{\mathbf{f}}=0
$$

where $\overline{\mathbf{w}}=[\overline{\mathbf{u}}, \overline{\mathbf{v}}, \bar{\sigma}]^{T}$ is a state variable containing the components of displacement, velocity and stress, and the vector components of $\overline{\mathbf{f}}$ are given by $\overline{\mathbf{f}}_{i}=A_{i} \overline{\mathbf{w}}$. Here, $A_{i}$ are coefficient matrices whose components depend on the density of the solid $\bar{\rho}$ and on the Lamé parameters $\lambda$ and $\mu$. For a component grid defined by the (static) mapping $\overline{\mathbf{x}}=\overline{\mathbf{G}}(\mathbf{r})$, equation (37) becomes

$$
\partial_{t} \overline{\mathbf{w}}+\frac{1}{\bar{J}} \nabla_{\mathbf{r}} \cdot \overline{\mathbf{F}}=0
$$

where $\bar{J}=\left|\overline{\mathbf{x}}_{\mathbf{r}}\right|$ is the Jacobian of the mapping (similar to before) and the vector components of $\overline{\mathbf{F}}$ are given by

$$
\overline{\mathbf{F}}_{i}=J C_{i} \overline{\mathbf{w}}, \quad C_{i}=\sum_{j=1}^{n_{d}} \frac{\partial \bar{r}_{i}}{\partial x_{j}} A_{j}, \quad i=1, \ldots, n_{d} .
$$

Our numerical approach for solving the mapped equations in (38) uses a second-order (Godunov-based) upwind scheme. The approach is similar to that discussed in [19] except that a stress-strain relaxation term is added to the evolution equation for the stress in (2), giving the modified stress equation

$$
\partial_{t} \overline{\boldsymbol{\sigma}}=S\left(\nabla_{\overline{\mathbf{x}}} \overline{\mathbf{v}}\right)-\tilde{\beta}\left(\overline{\boldsymbol{\sigma}}-S\left(\nabla_{\overline{\mathbf{x}}} \overline{\mathbf{u}}\right)\right) .
$$

Recall that $\bar{\sigma}=\mathcal{S}\left(\nabla_{\overline{\mathbf{x}}} \overline{\mathbf{u}}\right)$ is the stress-strain relationship as defined in Section 3. The quantity $S\left(\nabla_{\overline{\mathbf{x}}} \overline{\mathbf{u}}\right)$ in (39) is discretized using fourth-order centered differences while the parameter $\tilde{\beta}$ is taken to be proportional to the reciprocal of the time step, $\tilde{\beta}=\beta / \Delta t$. At the continuous level, the stress-strain relaxation term is identically zero. However, at the discrete level, this term helps to ensure that the stress-strain relation remains nearly satisfied even for long-time integrations. In general, we observe that the numerical errors in the components of stress are reduced when the stress-strain relaxation term is used. The numerical approximation for the elasticity equations is adjusted to ensure free-stream preservation. The overall FSI-DCG approximation is also free-stream preserving.

\subsection{Multi-domain time-stepping algorithm}

We use a strongly coupled partitioned approach to time step the numerical solutions on the fluid and solid domains. There is a separate fluid dynamics domain solver ( $\mathrm{ggcns}$ ) for each fluid domain and a separate solid mechanics domain solver (Cgsm) for each solid domain. Thus, if there is one fluid domain and two solid domains (as in Figure 14), then there will be one instance of Cgcns and two instances of Cgsm. As a result of this separation, each fluid or solid in its respective domain may have independent material parameters and constitutive laws. We note also that the geometry of any given domain does not need to be simply connected and thus a single domain may consist of, and a single domain solver can be used for, multiple disjoint regions if desired. A multi-physics control program (Cgmp) manages the multi-domain time stepping. At each time step, Cgmp calls the separate domain solvers to advance the solutions in their respective domains. Cgmp also manages the communication of interface data between the domain solvers and manages the assignment of the interface conditions.

In Section 4.2 we presented our FSI-DCG time-stepping algorithm in detail for the one-dimensional elastic-piston problem. The goal here is to describe an extension of this algorithm to the more general multi-dimensional, multi-domain case.

A major change for the multi-dimensional FSI-DCG algorithm is the need to call the hyperbolic grid generator and overlapping grid generator to update the mesh as the geometry evolves. This additional step is required in the Interior(a) stage of the algorithm in Figure 4. Given the predicted values for the points on an interface, a curve is fit to these points (we use a cubic-spline or NURBS curve). This interface curve is used as the starting curve for the hyperbolic grid generator which marches a given number of steps into 
the domain to define a grid near the interface at the new time. In this approach only the grid points on the interface are directly evolved in time. We note, however, that there are many other possible schemes that could be used for regenerating the deformed grid. For example, all of the grid points belonging to a deforming grid could be evolved using some appropriate evolution equations. We have not attempted this approach here although it might be useful in general. Once the new interface grids have been determined, the overlapping grid generator, Ogen, is called to update the grid connectivity. It is important to note that since the governing equations (35)-(36) for the fluid are solved in conservation form in a coordinate system that moves with the grid, it is not necessary to remap (interpolate) the solution from the deformed grid at the old time to that at the new time, thus avoiding a potentially expensive remapping step. The solution values on the new grid are automatically determined in a conservative fashion through the equations (35)-(36) which take into account the motion and deformation of an evolving fluid grid cell. In contrast, many ALE schemes perform one or more Lagrange steps (when the grid follows the fluid trajectories) followed by an Eulerian remap step where the solution from the Lagrange steps must be remapped (interpolated) to a new (usually better quality) grid.

At the Interior(b) stage of the FSI-DCG algorithm in Figure 4, the values for $\mathbf{w}_{\mathbf{i}}^{n}$ and $\overline{\mathbf{w}}_{\mathbf{i}}^{n}$ are computed at all grid points in the interior and on the interface of each fluid and solid domain. For the multi-dimensional case, this is done using discretizations of the governing equations in (35) and (38). The calculation of the projected values $\left[v_{I}, \sigma_{I}, \rho_{I}\right]$ listed in the Interface(a) stage of the figure is done using the previous formulas for the one-dimensional case, but with $v$ and $\sigma$ re-interpreted as $\mathbf{n} \cdot \mathbf{v}$ and $\mathbf{n} \cdot \sigma \cdot \mathbf{n}$, respectively, for the multi-dimensional case. The tangential component of the traction on the interface, $\mathbf{n} \cdot \sigma \cdot \boldsymbol{\tau}$, is zero from $(3)$ since the fluid is inviscid and only supports a stress on the interface in the normal direction due to the fluid pressure. These projected values of velocity, stress and density are used in the Interface(b) stage to update the appropriate components of the corresponding grid values in the fluid and the solid on the interface. In the multi-dimensional case we do not project the interface positions as in (25) but instead take the interface location determined by the solid for simplicity. This alternate approach does not present any difficulties since the displacement on the interface is determined from the time integral of the projected interface velocity.

The remaining stages of the FSI-DCG algorithm involve setting ghost points near the interface for the grids representing the fluid and solid domains. This is done following the stages listed in Figure 4 , but with some modifications needed to handle the governing equation in multiple space dimensions given in (35) and (38). For example, ghost points are extrapolated along the coordinate line that extends into the interior, using the limited extrapolation described in Section 4.2.4. Another modification involves the use of the interior equations on the boundary to determine values at ghost points (27). In multiple space dimensions the normal component of the momentum equation for the fluid is

$$
\mathbf{n} \cdot \mathbf{v}_{t}+\mathbf{n} \cdot((\mathbf{v} \cdot \nabla) \mathbf{v})+\frac{1}{\rho} \frac{\partial p}{\partial n}=0
$$

The interface condition on the velocity is

$$
\mathbf{n}(\mathbf{x}, t) \cdot \mathbf{v}(\mathbf{x}, t)=v_{I}(\mathbf{x}, t), \quad \text { for } \mathbf{x} \in \mathcal{I},
$$

and taking the time derivative of this last equation gives

$$
\mathbf{n} \cdot \mathbf{v}_{t}=\dot{v}_{I}-\mathbf{n}_{t} \cdot \mathbf{v}
$$

Using (41) in (40) gives the equation used to determine the pressure at the ghost points,

$$
\frac{\partial p}{\partial n}=-\rho\left(\dot{v}_{I}-\mathbf{n}_{t} \cdot \mathbf{v}+\mathbf{n} \cdot((\mathbf{v} \cdot \nabla) \mathbf{v})\right), \quad \text { for } \mathbf{x} \in \mathcal{I}
$$

This equation is a multi-dimensional version of the one for $\partial_{t} v$ in (27). The normal derivative of the pressure in (42) is seen to depend on terms involving the acceleration of the interface in the normal direction, a rotational acceleration, and an acceleration due to curvature effects. The latter two terms are extensions for the multi-dimensional case. We note that the rotational acceleration term $\mathbf{n}_{t} \cdot \mathbf{v}$ in (42) was missing in the compatibility boundary condition given in [22]. We also note that while the normal components of the equations, such as (40), are used on the interface to determined ghost point values, the tangential components of these same equations have also been applied on the interface when the interior equations were advanced in stage Interior(a). 


\section{Numerical results}

In this section we verify the accuracy and stability of the full FSI-DCG scheme for a number of steady and unsteady problems in two space dimensions. We begin in Section 6.1 by revisiting the elastic piston problem, but now we solve the problem on a two-dimensional domain. The results of this problem can be compared with the corresponding ones in Section 4.3 obtained using the MATLAB test code. Next, we consider the superseismic shock problem in Section 6.2. This problem has been considered by others as a verification test (see [8] and [39] for example), and the results computed here are compared with an exact similarity solution. In Section 6.3, a new deforming diffuser problem is described. This problem consists of a compressible gas flowing in a deformable expanding channel. A semi-analytical solution may be found for this smooth steady flow, and then used to demonstrate second-order accuracy in the max-norm for the full two-dimensional FSI-DCG approach. In Section 6.4, we show numerical results for an elastic shock hitting a fluid-filled cylindrical cavity. The solution of this problem is computed for a wide range of density (impedance) ratios, thus demonstrating the robust nature of our interface approximations in two dimensions. Finally, we consider the problem of a shock hitting two deformable sticks in Section 6.5. This problem illustrates, for a more complex problem, the ability of the FSI-DCG approach to treat problems with large displacements while retaining high quality grids. We note that all simulations to follow use the linearized approximation (23) to the fluid-solid Riemann problem.

\subsection{The two-dimensional elastic piston}

The elastic piston problem presented in Section 4 is solved using the FSI-DCG approach in two space dimensions. We consider a solid reference domain on $[-1,0] \times[0,1]$ and a fluid domain initially on $[0,1.5] \times$ $[0,1]$. The composite grid for the domain is denoted by $\mathcal{G}_{e p}^{(j)}$, where $j$ denotes the grid resolution. The grid spacing in the $x$-direction is chosen to be $\Delta x^{(j)}=1 /(10 j)$. The spacing in the $y$-direction is held fixed at $\Delta y=2 / 10$. The composite grid $\mathcal{G}_{e p}^{(j)}$ consists of a static rectangular grid $\mathcal{R}_{s}$ for the solid domain $[-1,0] \times[0,1]$, a static background rectangular grid $\mathcal{R}_{f}$ on $[-.75,1.5] \times[0,1]$ for the fluid along with a deforming hyperbolic grid of normal width 0.5 next to the interface. The fluid domain is represented by the hyperbolic grid and the background grid which overlap where they meet. Figure 15 shows the grid $\mathcal{G}_{e p}^{(2)}$ at two times during a calculation in which the interface moves to the left. At each time step, the fluid interface moves and the hyperbolic grid generator is called to regenerate the deforming grid near the fluid interface. The grid for the solid is static, but is shown adjusted for displacement in the figure.

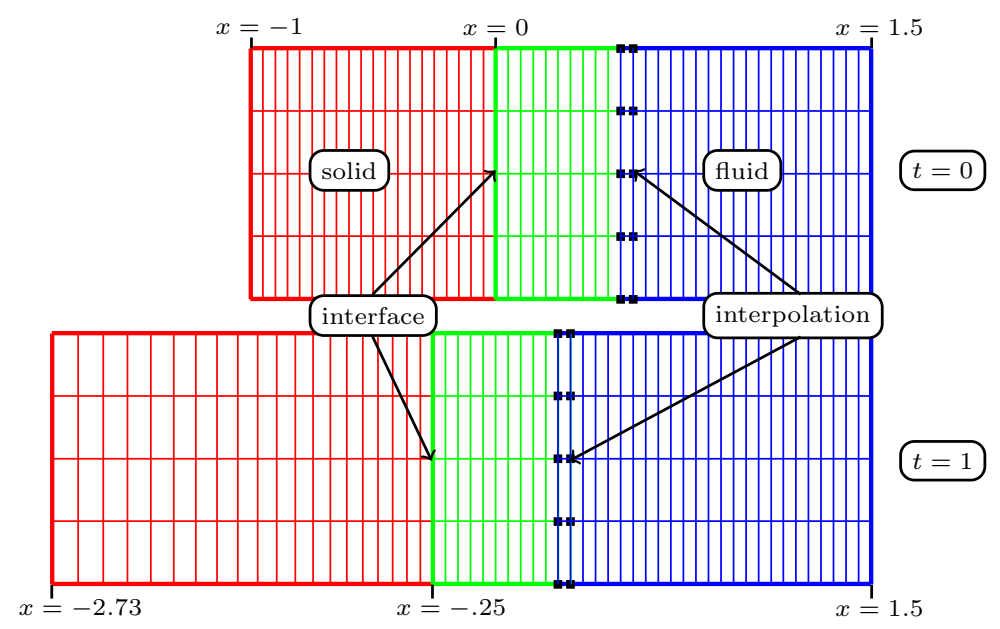

Figure 15: The composite grid $\mathcal{G}_{e p}^{(2)}$ for the two-dimensional elastic piston problem at times $t=0$ (top) and $t=1$ (bottom). The fluid domain is covered by a blue background grid and green grid that follows the interface. The red grid for the solid domain is shown adjusted for the displacement.

As a representative calculation, we consider the case of a smoothly receding interface whose solution is given in Appendix A. We take the parameters $\gamma=1.4, \rho_{0}=0.1, p_{0}=\rho_{0} / \gamma, \bar{\rho}=1, \lambda=1, \mu=1$, and choose $q=4$ in the definition (A.7) of the position of the interface. Note the impedance values $\bar{\rho} c_{p}=\sqrt{3}$ and $\rho_{0} a_{0}=0.1$. Initial conditions are taken from the exact solution. In addition, fluid grids at time $t=-\Delta t$ and $-2 \Delta t$ are provided as part of the initial conditions so that the initial grid velocities and boundary 
accelerations can be computed accurately using finite differences in time following the approach described in Section 5.1. Max-norm errors at $t=0.7$ are computed for a sequence of grid resolutions and the results are given in Figure 16. (The max-norm error of a vector grid function such as $\mathbf{v}_{\mathbf{i}}$, denoted by $\mathcal{E}_{\mathbf{v}}^{(\infty)}$, is defined as the maximum error over all active grid points and all components of the vector.) The max-norm errors are seen to converge at close to second-order accuracy.

\begin{tabular}{|l|c|c|c|c|c|c|c|c|c|c|c|c|}
\hline & \multicolumn{9}{|c|}{ Fluid } & \multicolumn{6}{|c|}{ Solid } \\
\hline Grid & $\mathcal{E}_{\rho}^{(\infty)}$ & $\mathrm{r}$ & $\mathcal{E}_{\mathbf{v}}^{(\infty)}$ & $\mathrm{r}$ & $\mathcal{E}_{T}^{(\infty)}$ & $\mathrm{r}$ & $\mathcal{E}_{\overline{\mathbf{u}}}^{(\infty)}$ & $\mathrm{r}$ & $\mathcal{E}_{\overline{\mathbf{v}}}^{(\infty)}$ & $\mathrm{r}$ & $\mathcal{E}_{\bar{\sigma}}^{(\infty)}$ & $\mathrm{r}$ \\
\hline $\mathcal{G}_{e p}^{(4)}$ & $3.7 \mathrm{e}-5$ & & $4.0 \mathrm{e}-4$ & & $2.5 \mathrm{e}-4$ & & $4.4 \mathrm{e}-5$ & & $2.8 \mathrm{e}-5$ & & $4.2 \mathrm{e}-5$ & \\
\hline $\mathcal{G}_{e p}^{(8)}$ & $8.0 \mathrm{e}-6$ & 4.7 & $8.0 \mathrm{e}-5$ & 4.9 & $4.9 \mathrm{e}-5$ & 5.1 & $1.2 \mathrm{e}-5$ & 3.6 & $5.6 \mathrm{e}-6$ & 4.9 & $7.5 \mathrm{e}-6$ & 5.7 \\
\hline $\mathcal{G}_{e p}^{(16)}$ & $2.2 \mathrm{e}-6$ & 3.6 & $2.2 \mathrm{e}-5$ & 3.6 & $1.0 \mathrm{e}-5$ & 4.9 & $3.2 \mathrm{e}-6$ & 3.8 & $1.2 \mathrm{e}-6$ & 4.8 & $1.4 \mathrm{e}-6$ & 5.4 \\
\hline $\mathcal{G}_{e p}^{(32)}$ & $5.9 \mathrm{e}-7$ & 3.8 & $5.9 \mathrm{e}-6$ & 3.8 & $2.2 \mathrm{e}-6$ & 4.6 & $8.2 \mathrm{e}-7$ & 3.9 & $2.6 \mathrm{e}-7$ & 4.6 & $3.1 \mathrm{e}-7$ & 4.5 \\
\hline rate & 1.99 & & 2.01 & & 2.27 & & 1.91 & & 2.25 & & 2.37 & \\
\hline
\end{tabular}

Figure 16: Two-dimensional elastic piston. Max-norm errors at $t=0.7$ for a smoothly receding piston.

\subsection{The superseismic shock}

The superseismic-shock problem consists of a shock in a gas that travels at an oblique angle to a planar solid surface. The elevated pressure behind the shock causes the solid to deflect and generate traveling p-wave and s-wave "shocks" in the solid. The flow is steady in a reference frame attached to the junction of the fluid shock and the p- and s-wave shocks in the solid, and an exact similarity solution may be constructed analytically (see Appendix C). Figure 17 illustrates one solution configuration in the reference frame fixed with the pre-shocked solid. In this reference frame, the junction of the shocks propagates to the right at a

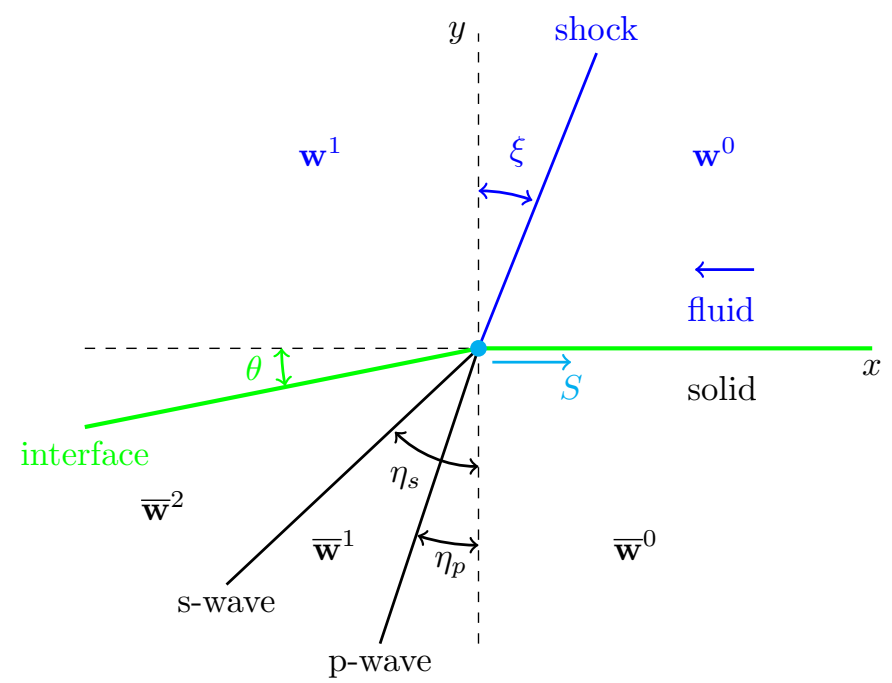

Figure 17: Flow structure for the superseismic shock problem showing the fluid shock, solid p-wave, solid s-wave and the fluid-solid interface. The pattern propagates to the right at speed $S$.

speed $S$. For the fluid, we let $\mathbf{w}^{0}=\left[\rho^{0}, v_{1}^{0}, v_{2}^{0}, p^{0}\right]$ and $\mathbf{w}^{1}=\left[\rho^{1}, v_{1}^{1}, v_{2}^{1}, p^{1}\right]$ denote the states ahead of and behind the shock, respectively. For the solid, let $\overline{\mathbf{w}}^{k}=\left[\overline{\mathbf{u}}^{k}, \overline{\mathbf{v}}^{k}, \overline{\boldsymbol{\sigma}}^{k}\right], k=0,1,2$, denote the state ahead of the p-wave $(k=0)$, the state between the $\mathrm{p}$ - and s-waves $(k=1)$, and the state behind the s-wave $(k=2)$. A slip between the fluid and solid velocities in the upstream state is allowed so that $v_{1}^{0}$ need not be zero. This upstream slip is introduced in order to produce a solution with no slip in the post-shock region. We have found that a strong slip in the post-shock region can enhance the growth of physical interface instabilities and cause the formation of small Scholte waves (a Scholte wave [40] is a wave that travels along the interface between a solid and a fluid) and thus we consider a solution with no post-shock slip in order to avoid the appearance of such waves.

We consider an exact solution of the superseismic-shock problem for a fluid with $\gamma=1.4$ and initial state $\left[\rho^{0}, v_{1}^{0}, v_{2}^{0}, p^{0}\right]=[0.1,-1.812303,0,0.02]$, and for a solid with parameters $[\bar{\rho}, \lambda, \mu]=[5,1, .5]$ and zero initial state $\overline{\mathbf{w}}^{0}=\left[\overline{\mathbf{u}}^{0}, \overline{\mathbf{v}}^{0}, \overline{\boldsymbol{\sigma}}^{0}\right]=\mathbf{0}$. When the shock junction moves with speed $S=.7$, the shock angle is found to be $\xi=.05894547$ (to the number of digits shown). From these parameters, the solution values for the other 
fluid and solid states can be computed directly using the formulae in Appendix C. For reference we provide these values here (to 4 significant figures). The post-shock fluid state is

$$
\left[\rho^{1}, v_{1}^{1}, v_{2}^{1}, p^{1}\right]=[.4908, .1811,-.1176, .5208] .
$$

The solid states are given by

$$
\begin{array}{ll}
\overline{\mathbf{u}}^{1}=-\alpha_{p}\left(\boldsymbol{\kappa}^{p} \cdot \overline{\mathbf{x}}-c_{p} t\right) \mathbf{a}^{p}, & \overline{\mathbf{u}}^{2}=\overline{\mathbf{u}}^{1}-\alpha_{s}\left(\boldsymbol{\kappa}^{s} \cdot \overline{\mathbf{x}}-c_{s} t\right) \mathbf{a}^{s}, \\
\overline{\mathbf{v}}^{1}=\alpha_{p} c_{p} \mathbf{a}^{p}, & \overline{\mathbf{v}}^{2}=\overline{\mathbf{v}}^{1}+\alpha_{s} c_{s} \mathbf{a}^{s} .
\end{array}
$$

with $c_{p}=.6325, c_{s}=.3162, \alpha_{p}=.3736, \alpha_{s}=-.1146, \boldsymbol{\kappa}^{p}=\mathbf{a}^{p}=[.9035,-.4286], \boldsymbol{\kappa}^{s}=[.4518,-.8921]$ $\left(\mathbf{a}^{s}=\left[-\kappa_{2}^{s}, \kappa_{1}^{s}\right]\right)$. The solid stress states $\overline{\boldsymbol{\sigma}}^{1}$ and $\overline{\boldsymbol{\sigma}}^{2}$ can be computed from the corresponding displacement states (43) using the stress-strain relationship. The interface deflection angle is $\theta=.2230$ while the p- and s-wave angles are $\eta_{p}=.4429$ and $\eta_{s}=1.102$, respectively.
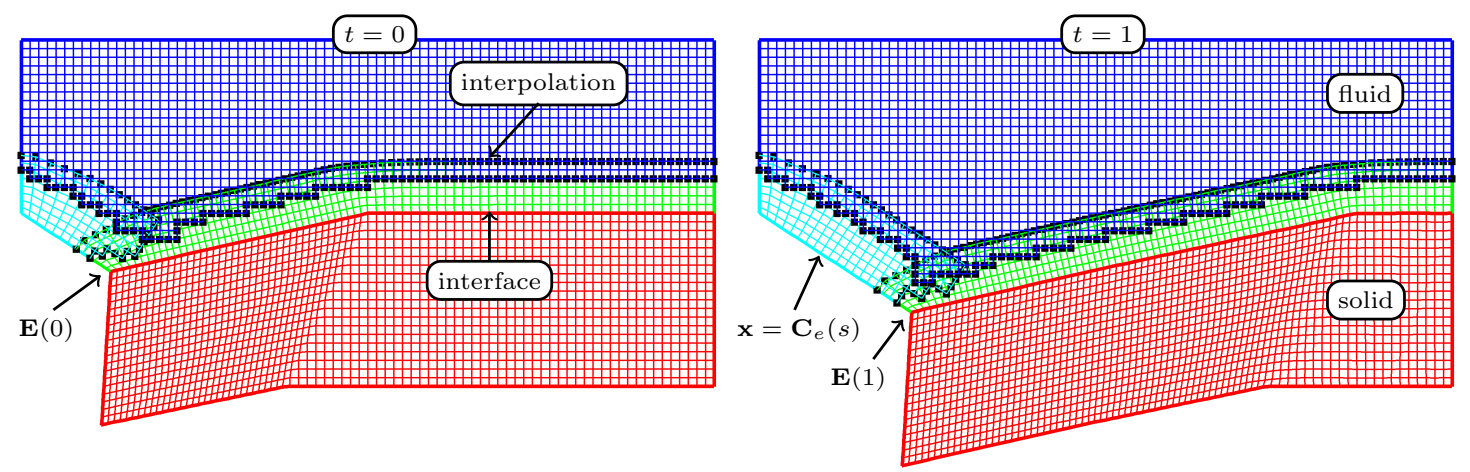

Figure 18: The overlapping grid $\mathcal{G}_{s s}^{(4)}$ for the superseismic shock problem. The grid is shown at times $t=0$ and $t=1$. The overlapping grid for the fluid domain consists of the blue, green and cyan grids. The green interface grid deforms to match the interface. The blue and cyan grids are static. The red grid for the solid domain is shown adjusted for the displacement.

Numerical solutions to the superseismic problem may be obtained using the FSI-DCG approach. This is done by performing computations in the reference frame fixed with the pre-shock solid as shown in Figure 17 . The composite grid for this calculation is denoted by $\mathcal{G}_{s s}^{(j)}$, where $j$ denotes the grid resolution with grid spacing equal to $\Delta s^{(j)}=1 /(10 j)$ approximately. The grid $\mathcal{G}_{s s}^{(4)}$ is shown in Figure 18 at $t=0$ and $t=1$. The grid at $t=0$ is constructed based on the initial state given by the exact solution, while the grid at $t=1$ is determined by the numerical solution at that time. The overlapping grid for the fluid domain consists of three component grids. A blue background Cartesian grid for the rectangle $[-1,1] \times[-.35, .5]$, a green hyperbolic grid of normal width $5 \Delta s^{(j)}$ adjacent to the interface, and a cyan hyperbolic grid with normal width $7 \Delta s^{(j)}$ adjacent to the end curve $\mathbf{C}_{e}$. The green hyperbolic grid deforms with the interface as the solution evolves in time, whereas the other two fluid grids are static. Grid points on these latter two static grids near the overlap with the deforming grid become exposed in time as the interface deflects downward. The end curve $\mathbf{x}=\mathbf{C}_{e}(s)$ is a straight line that follows the exact motion of the material interface point $\mathbf{E}(t)$ shown in Figure 18. As time evolves, $\mathbf{E}(t)$ moves downward and to the right as the interface deflects. The grid for the reference solid domain consists of the red Cartesian grid for the rectangle $[-1,1] \times[-.5, .0]$. The solid grid shown in the figure is adjusted for the computed displacement.

The numerical calculation requires initial conditions and boundary conditions. Initial conditions for the fluid and solid domains are taken from the exact solution. In addition, the fluid grids at time $t=-\Delta t$ and $-2 \Delta t$ are provided as part of the exact initial conditions so that the initial grid velocities and boundary accelerations can be accurately computed using finite differences in time following the approach described in Section 5.1. The conditions at the fluid-solid interface are computed using the FSI-DCG approach, whereas the boundary conditions on the perimeter of the fluid and solid domains are taken as Dirichlet conditions using the exact solution in time.

Figure 19 shows shaded contours of the solution to the superseismic shock problem at $t=1$ computed using the grid $\mathcal{G}_{s s}^{(64)}$. (This grid with $\Delta s^{(64)}=1 / 640$ has a total of about $1.1 \times 10^{6}$ grid points.) The numerical solution in the solid is shown in the deformed space $\mathbf{x}=\overline{\mathbf{x}}+\overline{\mathbf{u}}$. Note that the s-wave angle $\eta_{s}=1.102$ in the undeformed space becomes $\widetilde{\eta_{s}}=.8947$ in the deformed space. The shock in the fluid, the p- and s-wave shocks in the solid, and the fluid-solid interface are clearly visible in the shaded contour plot. Also evident 
are usual artifacts in the fluid density that are due to the initial conditions and a slight mismatch between the solution and exact boundary condition at the upper wall, together with small reflections in the solid where the p-wave intersects the lower boundary. Figure 20 presents the discrete $L_{1}$-norm errors and estimated convergence rates for a sequence of grids of increasing resolution. The discrete $L_{p}$-norm for a grid function $v_{\mathbf{i}}$ is defined in terms of the solution values at all active grid points divided by the total number of active points, $\mathrm{N}$,

$$
\left\|v_{\mathbf{i}}\right\|_{p}=\left[\frac{1}{N} \sum_{\mathbf{i}}\left|v_{\mathbf{i}}\right|^{p}\right]^{1 / p} .
$$

For the error in (possibly) vector and matrix-valued grid functions, we take the error to be the maximum, over all components, of the $L_{1}$-norm errors of the individual components, for example

$$
\mathcal{E}_{\mathbf{v}}^{(1)}=\max \left\{\left\|v_{1, \mathbf{i}}-v_{1, \mathbf{i}}^{e}\right\|_{1},\left\|v_{2, \mathbf{i}}-v_{2, \mathbf{i}}^{e}\right\|_{1}\right\},
$$

where $v_{1, \mathbf{i}}$ and $v_{2, \mathbf{i}}$ are the two components of the vector-valued grid function $\mathbf{v}_{\mathbf{i}}$, and the corresponding quantities with superscript $e$ are the exact values on the grid. For the solid domain, the estimated $L_{1}$ norm convergence rates are close to the expected values of $4 / 3$ for $\mathcal{E}_{\overline{\mathbf{u}}}^{(1)}$, and of $2 / 3$ for $\mathcal{E}_{\overline{\mathbf{v}}}^{(1)}$ and $\mathcal{E}_{\overline{\boldsymbol{\sigma}}}^{(1)}$. The convergence rates for the fluid variables $\left(\mathcal{E}_{\rho}^{(1)}, \mathcal{E}_{\mathbf{v}}^{(1)}, \mathcal{E}_{T}^{(1)}\right)$ are all equal to 1 approximately. A convergence rate of 1 would be the expected rate for the computation of an isolated fluid shock and indicates that the slower convergence rate of $\mathcal{E}_{\overline{\mathbf{v}}}^{(1)}$ and $\mathcal{E}_{\bar{\sigma}}^{(1)}$ in the solid does not seem to degrade the convergence rate of the fluid variables. This is not so surprising given that the errors are largest at the solution discontinuities and not at the interface.

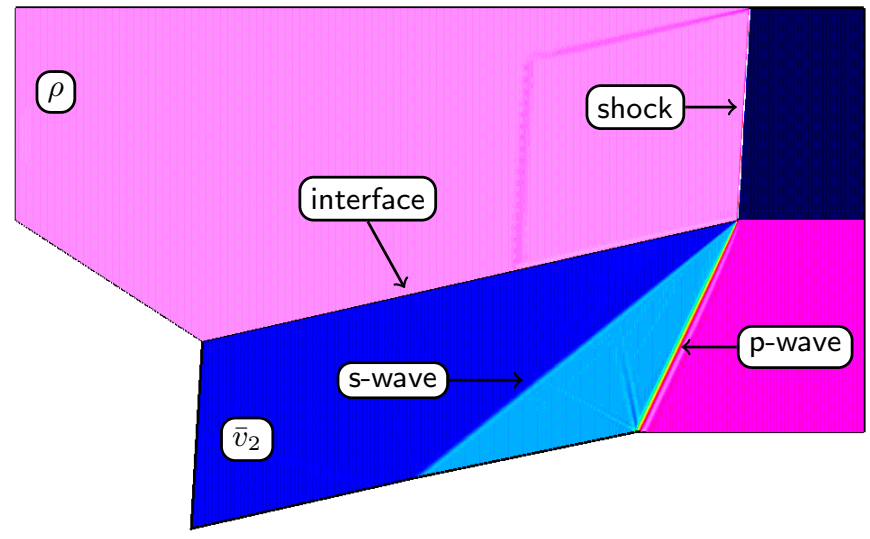

Figure 19: The superseismic shock solution computed on grid $\mathcal{G}_{s s}^{(64)}$ showing contours at $t=1.0$ of the density, $\rho$, in the fluid domain and the vertical velocity, $\bar{v}_{2}$, in the solid domain.

\begin{tabular}{|l|c|c|c|c|c|c|c|c|c|c|c|c|}
\hline & \multicolumn{9}{|c|}{ Solid } & \multicolumn{6}{c|}{ Fluid } \\
\hline Grid & $\mathcal{E}_{\overline{\mathbf{u}}}^{(1)}$ & $\mathrm{r}$ & $\mathcal{E}_{\overline{\mathbf{v}}}^{(1)}$ & $\mathrm{r}$ & $\mathcal{E}_{\overline{\boldsymbol{\sigma}}}^{(1)}$ & $\mathrm{r}$ & $\mathcal{E}_{\rho}^{(1)}$ & $\mathrm{r}$ & $\mathcal{E}_{\mathbf{v}}^{(1)}$ & $\mathrm{r}$ & $\mathcal{E}_{T}^{(1)}$ & $\mathrm{r}$ \\
\hline $\mathcal{G}_{s s}^{(4)}$ & $8.9 \mathrm{e}-4$ & & $6.4 \mathrm{e}-3$ & & $1.8 \mathrm{e}-2$ & & $5.9 \mathrm{e}-3$ & & $3.8 \mathrm{e}-2$ & & $1.2 \mathrm{e}-2$ & \\
\hline $\mathcal{G}_{s s}^{(8)}$ & $3.2 \mathrm{e}-4$ & 2.8 & $3.9 \mathrm{e}-3$ & 1.6 & $1.1 \mathrm{e}-2$ & 1.6 & $2.9 \mathrm{e}-3$ & 2.0 & $1.7 \mathrm{e}-2$ & 2.2 & $6.7 \mathrm{e}-3$ & 1.8 \\
\hline $\mathcal{G}_{s s}^{(16)}$ & $1.4 \mathrm{e}-4$ & 2.4 & $2.4 \mathrm{e}-3$ & 1.7 & $6.7 \mathrm{e}-3$ & 1.7 & $1.6 \mathrm{e}-3$ & 1.9 & $8.6 \mathrm{e}-3$ & 2.0 & $3.7 \mathrm{e}-3$ & 1.8 \\
\hline $\mathcal{G}_{s s}^{(32)}$ & $6.7 \mathrm{e}-5$ & 2.0 & $1.4 \mathrm{e}-3$ & 1.6 & $4.1 \mathrm{e}-3$ & 1.6 & $8.2 \mathrm{e}-4$ & 1.9 & $4.3 \mathrm{e}-3$ & 2.0 & $1.9 \mathrm{e}-3$ & 1.9 \\
\hline rate & 1.24 & & 0.72 & & 0.72 & & 0.94 & & 1.03 & & 0.88 & \\
\hline
\end{tabular}

Figure 20: Superseismic shock convergence results. Discrete $L_{1}$-norm errors at $t=1.0$.

\subsection{The deforming diffuser}

The deforming diffuser problem consists of a steady supersonic flow in a deformable expanding channel. We consider the flow in the vicinity of the lower wall of the channel as illustrated in Figure 21. The shape of the deformable wall is determined by a balance between the fluid pressure and the stresses in the solid. 
Ideally, the problem would be defined on a semi-infinite domain, but side boundaries are introduced for numerical convenience as shown in the figure. The geometry of the problem can be arranged so that the solution remains smooth with no shocks in the fluid domain and no corner-singularities in the solid. It is also possible to obtain a semi-analytical solution of the steady problem, and thus this solution can be used to check whether the computed FSI-DCG solution is second-order accurate in the max-norm for smooth two-dimensional flow.

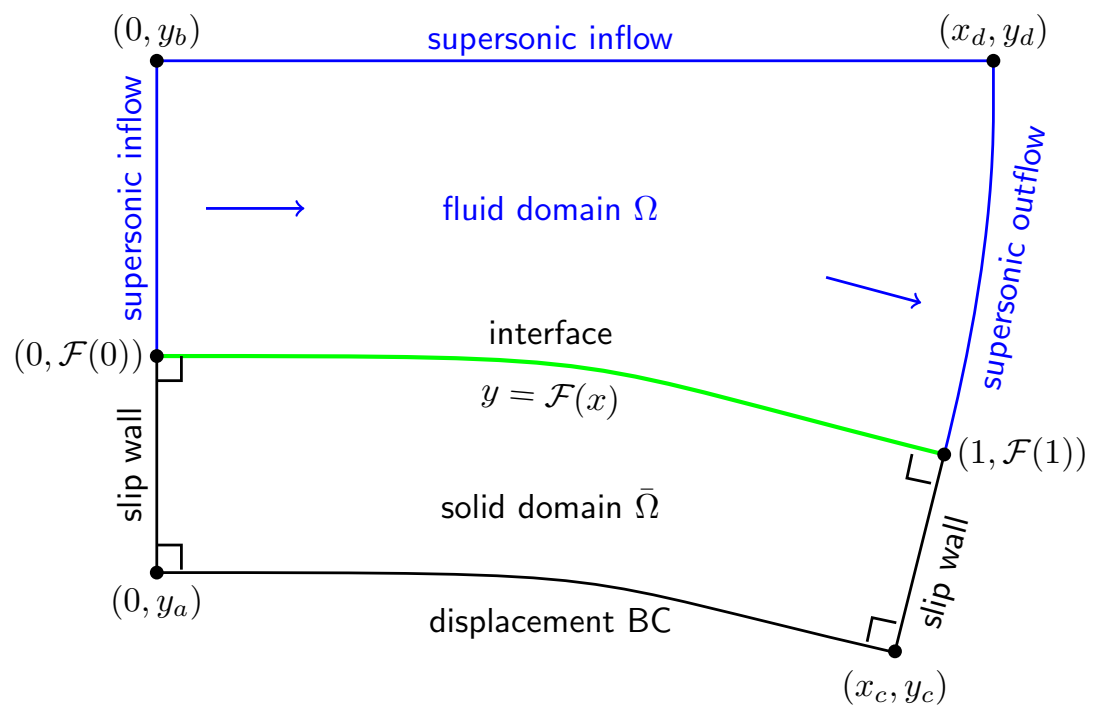

Figure 21: The geometry and boundary conditions for the deforming diffuser FSI problem. The right-angles indicated in the solid domain apply to the undeformed reference state.

We start by defining the geometry of the problem with reference to Figure 21 and later provide the actual values for the geometrical parameters that are used for the numerical results. Let $y=\mathcal{F}(x)$ denote the deformed shape of the wall for which the fluid and solid tractions balance exactly, and let $\bar{y}=\mathcal{F}_{0}(\bar{x})$ denote the shape of the wall in the reference (un-deformed) solid. The reference domain $\bar{\Omega}$ for the solid is thus bounded on the top by the curve $\bar{y}=\mathcal{F}_{0}(\bar{x}), \bar{x} \in[0,1]$. The sides of $\bar{\Omega}$ are straight lines which meet the two ends of the reference curve $\bar{y}=\mathcal{F}_{0}(\bar{x})$ at right angles. The bottom boundary of $\bar{\Omega}$ is defined by the cubic Hermite polynomial $\bar{y}=\mathcal{B}_{0}(\bar{x})$ that passes through the points $\left(0, y_{a}\right)$ and $\left(x_{c}, y_{c}\right)$ and meets the adjacent sides at right angles. The solid reference domain is chosen so that all corners are right angles. This, together with the choice of boundary conditions given below, means that potential corner singularities in the solid solution are avoided.

The fluid domain $\Omega$ is bounded by straight lines on the left and top and by $y=\mathcal{F}(x)$ on the bottom. The boundary curve on the right-hand side of $\Omega$ is defined as a smoothed version of the piecewise linear curve that joins the three points $(1, \mathcal{F}(1)),\left(x_{d}, y_{e}\right)$ and $\left(x_{d}, y_{d}\right)$, where $y_{e}=\mathcal{F}(1)-\left(x_{d}-1\right) / \mathcal{F}^{\prime}(1)$. The smoothed curve is defined using the integral of hyperbolic tangent functions (see [41] for full details). We call this smooth $\left(C^{\infty}\right)$ representation of a piecewise linear curve a smoothed polygon. The choice of this boundary curve is not particularly important since it does not significantly affect the results, provided the curve is sufficiently smooth.

The boundary conditions on the solid are taken as slip-wall on the left and right, and a displacement boundary condition $(\overline{\mathbf{u}}=0)$ on the bottom. The boundary conditions on the fluid are taken as steady supersonic inflow on the left with $(\rho, u, v, p)=\left(\rho_{0}, u_{0}, 0, p_{0}\right)$ (all variables given), supersonic inflow on the top (all variables set to the exact solution), and supersonic outflow on the right (all variables extrapolated to second order). The interface conditions in (3) are imposed on the expanding wall, $y=\mathcal{F}(x)$.

A semi-analytic solution to the deforming diffuser problem can be determined as follows. Given the fluid inflow conditions $\left(\rho_{0}, u_{0}, 0, p_{0}\right)$ and (the to be determined) interface curve, $y=\mathcal{F}(x)$, the solution in the fluid domain can by found using the method of characteristics and the well-known Prandtl-Meyer function (see for example [22] or [28] for further details). In particular, this solution gives an analytic expression for the fluid pressure on the interface as a function of the interface shape which we denote by $p=\mathcal{P}(x, \mathcal{F}(x))$. This relationship between the fluid pressure and the shape of the interface provides a nonlinear boundary condition for the solid in $\bar{\Omega}$. This boundary condition is

$$
\overline{\mathbf{n}}(\overline{\mathbf{x}}) \cdot \overline{\boldsymbol{\sigma}}(\overline{\mathbf{x}})=-\left(\mathcal{P}(\overline{\mathbf{x}}+\overline{\mathbf{u}}, \mathcal{F}(\overline{\mathbf{x}}+\overline{\mathbf{u}}))-p_{0}\right) \mathbf{n}(\overline{\mathbf{x}}+\overline{\mathbf{u}}), \quad \text { for } \overline{\mathbf{x}}=\left(\bar{x}, \mathcal{F}_{0}(\bar{x})\right) \text { with } \bar{x} \in(0,1) .
$$


Here $\overline{\mathbf{n}}$ is the normal to the solid reference domain (i.e. the normal to the curve $\bar{y}=\mathcal{F}_{0}(\bar{x})$ ) while $\mathbf{n}(\overline{\mathbf{x}}+\overline{\mathbf{u}})$ is the normal to the deformed fluid domain (i.e. the normal to the curve $y=\mathcal{F}(x)$ ). We now have a well-defined problem for the solid in $\bar{\Omega}$ consisting of the equations of steady elasticity with the boundary condition in (45) on $\bar{y}=\mathcal{F}_{0}(\bar{x})$ and the boundary conditions shown in Figure 21 on the remaining sides.

The boundary-value problem defined for the solid is solved numerically for the components of displacement using a second-order accurate (centered) finite-difference scheme. The equations are nonlinear due to the boundary condition in (45), and a method of iteration is needed to solve them. Using a provisional choice for the shape of the interface in (45), the (linear) equations can be solved for the components of displacement, which are then used to update the shape of the interface. Repeating this procedure defines a simple fixedpoint iteration which converges rapidly. The converged solution for the solid gives a discrete approximation for the interface curve, $y=\mathcal{F}(x)$, and this curve, in turn, specifies the solution in the fluid domain. We solve the boundary-value problem for the solid on a grid with $1280 \times 512$ points. This fine-grid solution for the solid domain, together with the analytic Prandtl-Meyer solution in the fluid domain, is taken as the exact solution of the deforming diffuser problem.

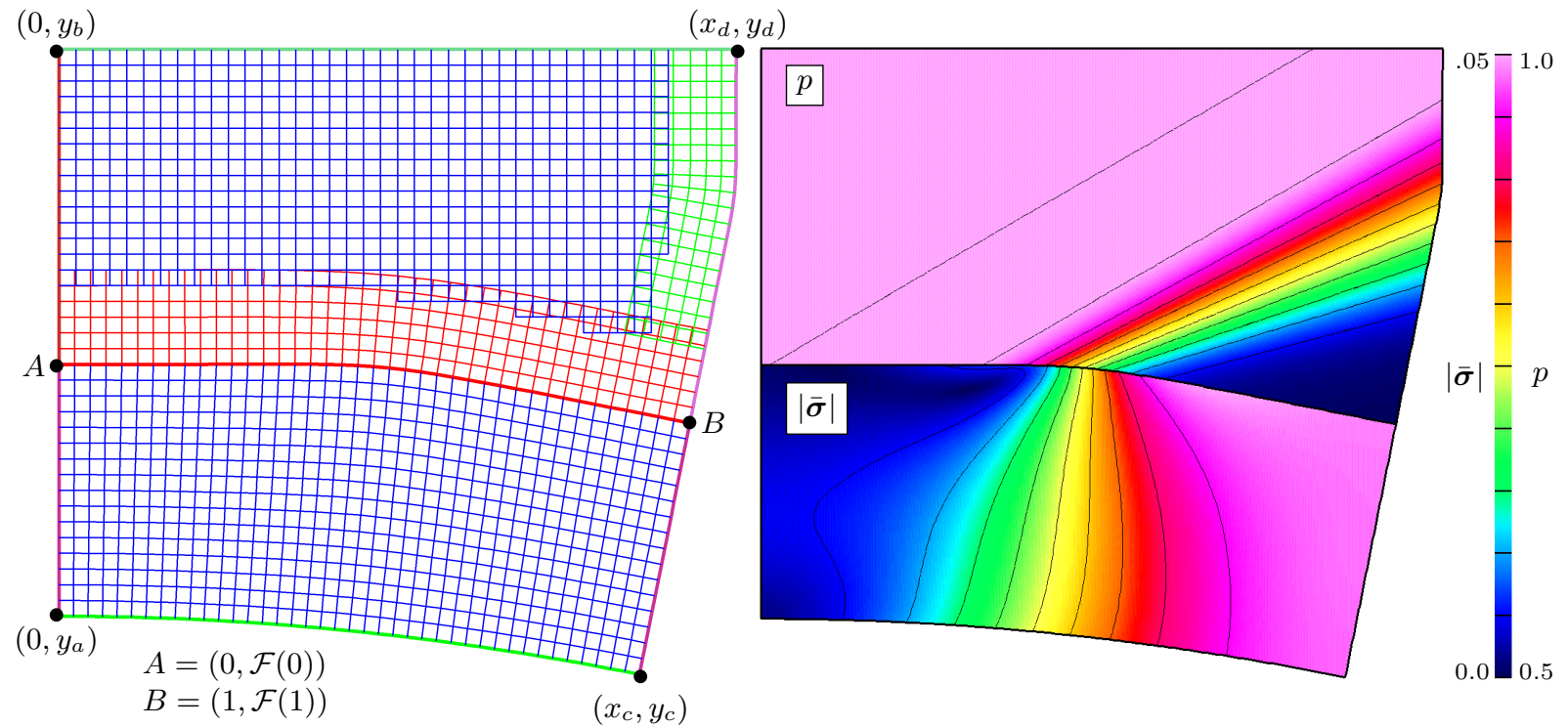

Figure 22: Left: Composite grid $\mathcal{G}_{d d}^{(4)}$ for the deforming diffuser problem showing the deformed grid in the lower solid domain. Right: computed solution on grid $\mathcal{G}_{d d}^{(16)}$ showing contours of the fluid pressure and norm of the solid stress tensor.

We now define the actual geometry and parameters used in the numerical computations. The reference (un-deformed) interface curve is taken as

$$
\bar{y}=\mathcal{F}_{0}(\bar{x})=\frac{m_{0}+m_{1}}{2}\left(\bar{x}-x_{0}\right)+\frac{m_{1}-m_{0}}{2 \beta} \log \left(2 \cosh \left(\beta\left(\bar{x}-x_{0}\right)\right)\right)
$$

for $0<\bar{x}<1$, where the initial and final slopes are $m_{0}=0$ and $m_{1}=-0.2$, respectively, and $x_{0}=.5$ and $\beta=10.0$. The thickness of the solid domain is taken to be $h_{s}=0.4$ which we use to define $y_{a}=-h_{s}$, $x_{c}=1+m_{1} h_{s} / \sqrt{1+m_{1}^{2}}$ and $y_{c}=\mathcal{F}_{0}(1)-h_{s} / \sqrt{1+m_{1}^{2}}$. The parameters that define the vertices of the fluid domain are taken to be $y_{b}=0.5, x_{d}=1.075$ and $y_{d}=0.5$. The parameters that define the gas flowing into the fluid domain are $\rho_{0}=1.4, u_{0}=2, p_{0}=1$ and $\gamma=1.4$, which corresponds to a supersonic flow with Mach number equal to 2 . For the solid we use $\bar{\rho}=\lambda=\mu=10$.

The composite grid for the deforming diffuser FSI problem is shown in Figure 22, Let $\mathcal{G}_{d d}^{(j)}$ denote the composite grid of resolution factor $j$ which has a grid spacing approximately equal to $\Delta s^{(j)}=1 /(10 j)$. The grid for the solid domain is defined by linear transfinite interpolation between the top and bottom boundary curves for $\bar{\Omega}$. The overlapping grid for the fluid domain consists of three component grids: a (deforming) hyperbolic grid with fixed normal distance of 0.1 for the region next to the interface, a (static) smoothed-polygon grid of normal distance 0.1 for the right end, and a (static) background Cartesian grid. The hyperbolic grid is generated with the hyperbolic grid generator as discussed Section 5.3. The smoothedpolygon grid is determined by extending normals from the (previously defined) smoothed-polygon curve for the right boundary. 
The initial conditions for the computations are taken from the exact (semi-analytic) solution and we integrate the equations to $t=1$. Note that time-dependent equations are solved in both the fluid and solid domains using our FSI-DCG approach, and no attempt is made to integrate to within a (small) specified tolerance of steady state as this could take an impractically long time. Figure 22 shows shaded contours of the computed solution at $t=1$ on grid $\mathcal{G}_{d d}^{(16)}$. The pressure of the gas decreases as it expands around the wall, and the straight contour lines indicate a simple-wave solution in agreement with the exact solution. The decreased pressure in the gas downstream of the bend in the wall results in an upward deflection of the fluid-solid interface. This upward deflection from the undeformed reference state creates a non-zero stress field in the solid as shown in the figure.

The maximum errors at $t=1$ are given in Figures 23 and 24 for two cases. The high-order Godunov based method for the fluid domain has a slope-limiter which can locally reduce the order of accuracy of the scheme in certain situations, and we present results with the limiter turned off and on. Figure 24 gives the max-norm convergence rates for the case when the slope-limiter is turned off. We note that the convergence rates are close to 2 for all of the fluid variables. The convergence rates for the velocity and stress in the solid are also 2, approximately, while the displacement appears to be converging at a rate somewhat higher than 2. Figure 24 shows the convergence rates when the slope-limiter is turned on, and the results indicate a lower rate of convergence for the fluid variables as expected. More refined studies of the fluid domain alone indicate a likely convergence rate of $4 / 3$ instead of 2 when the slope-limiter is on.

\begin{tabular}{|l|c|c|c|c|c|c|c|c|c|c|c|c|}
\hline & \multicolumn{9}{|c|}{ Solid } & \multicolumn{6}{|c|}{ Fluid } \\
\hline Grid & $\mathcal{E}_{\overline{\mathbf{u}}}^{(\infty)}$ & $\mathrm{r}$ & $\mathcal{E}_{\overline{\mathrm{v}}}^{(\infty)}$ & $\mathrm{r}$ & $\mathcal{E}_{\bar{\sigma}}^{(\infty)}$ & $\mathrm{r}$ & $\mathcal{E}_{\rho}^{(\infty)}$ & $\mathrm{r}$ & $\mathcal{E}_{\mathbf{v}}^{(\infty)}$ & $\mathrm{r}$ & $\mathcal{E}_{T}^{(\infty)}$ & $\mathrm{r}$ \\
\hline $\mathcal{G}_{d d}^{(2)}$ & $1.6 \mathrm{e}-4$ & & $2.8 \mathrm{e}-4$ & & $2.9 \mathrm{e}-2$ & & $3.4 \mathrm{e}-2$ & & $2.1 \mathrm{e}-2$ & & $7.0 \mathrm{e}-3$ & \\
\hline $\mathcal{G}_{d d}^{(4)}$ & $3.3 \mathrm{e}-5$ & 4.8 & $1.1 \mathrm{e}-4$ & 2.6 & $8.9 \mathrm{e}-3$ & 3.3 & $8.6 \mathrm{e}-3$ & 3.9 & $6.3 \mathrm{e}-3$ & 3.4 & $1.9 \mathrm{e}-3$ & 3.8 \\
\hline $\mathcal{G}_{d d}^{(8)}$ & $5.6 \mathrm{e}-6$ & 5.9 & $2.8 \mathrm{e}-5$ & 3.9 & $1.8 \mathrm{e}-3$ & 5.0 & $2.2 \mathrm{e}-3$ & 3.8 & $2.1 \mathrm{e}-3$ & 3.0 & $5.9 \mathrm{e}-4$ & 3.2 \\
\hline $\mathcal{G}_{d d}^{(16)}$ & $9.4 \mathrm{e}-7$ & 5.9 & $6.8 \mathrm{e}-6$ & 4.1 & $3.5 \mathrm{e}-4$ & 5.0 & $5.8 \mathrm{e}-4$ & 3.8 & $4.7 \mathrm{e}-4$ & 4.4 & $1.3 \mathrm{e}-4$ & 4.4 \\
\hline rate & 2.48 & & 1.81 & & 2.14 & & 1.95 & & 1.81 & & 1.88 & \\
\hline
\end{tabular}

Figure 23: Deforming diffuser convergence results. Maximum-norm errors at $t=1.0$ with the slope-limiter turned off in the fluid solver.

\begin{tabular}{|l|c|c|c|c|c|c|c|c|c|c|c|c|}
\hline & \multicolumn{9}{|c|}{ Solid } & \multicolumn{7}{|c|}{ Fluid } \\
\hline Grid & $\mathcal{E}_{\overline{\mathbf{u}}}^{(\infty)}$ & $\mathrm{r}$ & $\mathcal{E}_{\overline{\mathrm{v}}}^{(\infty)}$ & $\mathrm{r}$ & $\mathcal{E}_{\bar{\sigma}}^{(\infty)}$ & $\mathrm{r}$ & $\mathcal{E}_{\rho}^{(\infty)}$ & $\mathrm{r}$ & $\mathcal{E}_{\mathrm{v}}^{(\infty)}$ & $\mathrm{r}$ & $\mathcal{E}_{T}^{(\infty)}$ & $\mathrm{r}$ \\
\hline $\mathcal{G}_{d d}^{(2)}$ & $1.1 \mathrm{e}-4$ & & $2.4 \mathrm{e}-4$ & & $3.0 \mathrm{e}-2$ & & $4.3 \mathrm{e}-2$ & & $2.7 \mathrm{e}-2$ & & $8.8 \mathrm{e}-3$ & \\
\hline $\mathcal{G}_{d d}^{(4)}$ & $3.2 \mathrm{e}-5$ & 3.4 & $7.6 \mathrm{e}-5$ & 3.1 & $9.5 \mathrm{e}-3$ & 3.2 & $1.7 \mathrm{e}-2$ & 2.5 & $1.1 \mathrm{e}-2$ & 2.5 & $3.5 \mathrm{e}-3$ & 2.5 \\
\hline $\mathcal{G}_{d d}^{(8)}$ & $8.1 \mathrm{e}-6$ & 4.0 & $2.7 \mathrm{e}-5$ & 2.8 & $2.1 \mathrm{e}-3$ & 4.5 & $6.4 \mathrm{e}-3$ & 2.7 & $4.2 \mathrm{e}-3$ & 2.5 & $1.3 \mathrm{e}-3$ & 2.7 \\
\hline $\mathcal{G}_{d d}^{(16)}$ & $1.4 \mathrm{e}-6$ & 6.0 & $7.7 \mathrm{e}-6$ & 3.6 & $5.2 \mathrm{e}-4$ & 4.1 & $2.1 \mathrm{e}-3$ & 3.1 & $1.3 \mathrm{e}-3$ & 3.3 & $4.2 \mathrm{e}-4$ & 3.1 \\
\hline rate & 2.09 & & 1.64 & & 1.98 & & 1.46 & & 1.45 & & 1.46 & \\
\hline
\end{tabular}

Figure 24: Deforming diffuser convergence results. Maximum-norm errors at $t=1.0$ with the slope-limiter turned on in the fluid solver. The use of the slope-limiter reduces the convergence rates compared to the results with no limiter in Figure 23.

\subsection{An elastic shock impacting a deformable fluid cylinder}

The computations in this section evaluate the behavior of the FSI-DCG scheme for the simulation of an elastic shock impacting a fluid-filled deformable cylinder. The computation is performed for three different values of the solid density to demonstrate the stability of the interface approximation based on the fluidsolid Riemann problem for a range of impedance ratios and for a fully two-dimensional problem. The geometry of the problem, as shown in Figure 25, consists of a fluid-filled cylindrical cavity inside of a solid square. The cavity is centered at the origin and has a radius equal to 1 , while the solid square is located on $[-2.5,2.5] \times[-2.5,2.5]$. An elastic p-wave shock, initially at $x=-1.5$, travels from left to right, and the fluid inside the cavity is uniform initially. The boundary conditions for the solid are taken as Dirichlet on the left (all variables set to the traveling p-wave solution), slip walls on the top and bottom, and a displacement boundary on the right $(\overline{\mathbf{u}}=0)$. The interface conditions in (3) are applied at the fluid-solid interface. We note that fluid-solid interfaces can experience Rayleigh-Taylor like instabilities when a light material accelerates a heavy material [42, 43]. For the case considered here we choose a relatively weak 
elastic shock and integrate over a moderate time interval in order to avoid issues with the growth of true physical instabilities.
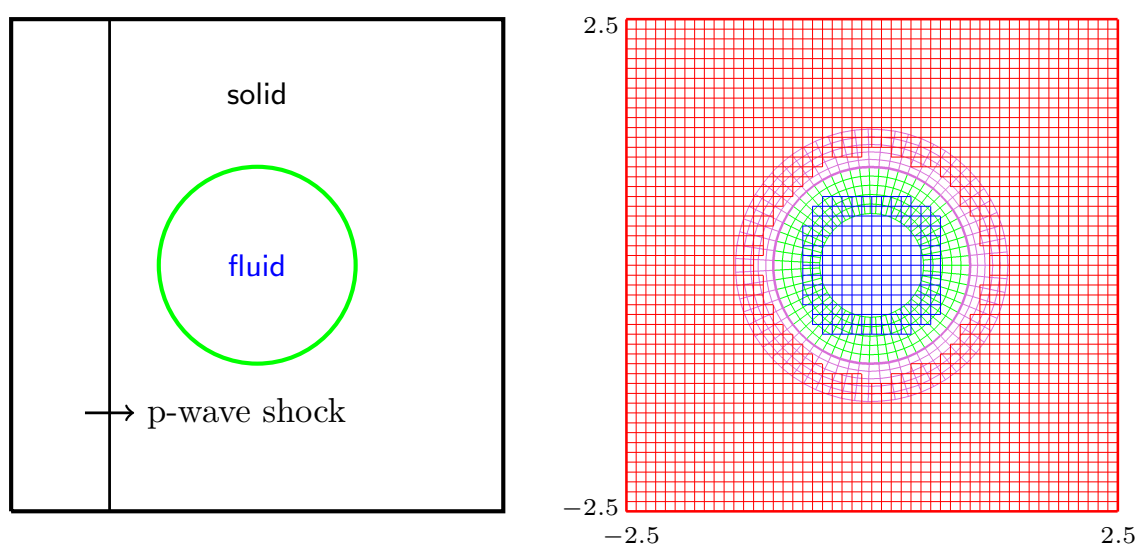

Figure 25: Left: geometry for the simulation of an elastic shock impacting a deforming fluid cylinder. Right: the composite grid $\mathcal{G}_{d c}^{(1)}$ showing the two solid grids and the two fluid grids.

Let $\mathcal{G}_{d c}^{(j)}$ denote the composite grid for the deforming cylinder problem with grid spacing approximately equal to $\Delta s^{(j)}=1 /(10 j)$, see Figure 25 . The outer solid domain is covered by a Cartesian grid on the square $[-2.5,2.5] \times[-2.5,2.5]$ and a (static) hyperbolic grid of width $4 \Delta s^{(j)}$ next to the interface. The inner fluid domain is covered by a Cartesian grid for the square $[-1.5,1.5] \times[-1.5,1.5]$ and a (deformable) hyperbolic grid of width $4 \Delta s^{(j)}$ adjacent the interface.

The material parameters for the solid are $\bar{\rho}=\lambda=\mu=\rho_{r}$, where $\rho_{r}$ is the initial solid-fluid density ratio, taken to be $\rho_{r}=0.1,1$ and 10 for the three cases considered. The initial conditions for the solid are taken as a traveling p-wave shock propagating in the positive $x$-direction. The elastic shock is described by (C.6)-(C.8) with $\boldsymbol{\kappa}^{p}=[1,0]^{T}, \alpha_{p}=0.1$ and $\alpha_{s}=0$, but then shifted so that the jump lies at $x=-1.5$ initially. The fluid is initially at rest with $\rho=1$ and $p=1 / \gamma$, where $\gamma=1.4$.

Figures $26-28$ show shaded contours of the stress-norm $|\overline{\boldsymbol{\sigma}}|=\sqrt{\bar{\sigma}_{11}^{2}+2 \bar{\sigma}_{12}^{2}+\bar{\sigma}_{22}^{2}}$ in the solid and $\rho$ in the fluid for the three cases at times $t=0,0.5$ and 1.0. The solutions shown in the figure were computed on the grid $\mathcal{G}_{d c}^{(32)}$ which has a total of $3.5 \times 10^{6}$ grid points approximately. Since the wave speeds in the solid are the same for all three cases, the incident p-wave travels the same distance for each case. The outermost reflected wave in the solid is a p-wave, which is clearly visible for each case, while there is a reflected s-wave lagging behind. This s-wave is most easily seen in the plots for $\rho_{r}=1$ and 10 . For the light-solid case, the interaction of the p-wave in the solid with the heavier fluid in the cavity generates small-amplitude acoustic waves in the fluid (as indicated by the small range of density represented by the colorbar). The cavity itself suffers only a small deflection as a result of the interaction. For the medium-solid and heavy-solid cases, the interaction with the fluid-filled cavity is stronger leading to the formation of a shock in the fluid. The compression of the cavity and the elevated pressure behind the shock lead to a lateral bulging of the cavity and an increase in stress in the solid in the vicinity of lateral sides of the cavity. This is seen most clearly in the heavy-solid case at $t=1.5$.

It is worth noting that computations of the low and medium-density solid cases, $\rho_{r}=0.1$ and $\rho_{r}=1$, both fail due to numerical instabilities when the standard velocity-from-solid/stress-from-fluid interface approach is used. In contrast, the calculations presented here use the interface approximation based on the fluid-solid Riemann problem and no numerical instability is observed.

To estimate the accuracy of the computed solution, we solve the problem on a sequence of grids of increasing resolution. Given solutions on three grids of increasing resolution, a posteriori estimates of the error and convergence rates can be computed using the procedure described in [32]. Estimated $L_{1}$-norm errors and convergence rates are given in Figure 29 for the medium-density solid case $\rho_{r}=1$ (similar results are obtained for the light and heavy cases). The rates are computed using the grids $\mathcal{G}_{d c}^{(4)}, \mathcal{G}_{d c}^{(8)}$ and $\mathcal{G}_{d c}^{(64)}$. Having the last grid significantly finer than the previous grids generally provides better estimated convergence rates for wave propagation problems with linear discontinuities in which solutions converge slowly, as is the case here. As seen from the figure, convergence rates of approximately 0.7 for $\mathcal{E}_{\overline{\mathbf{v}}}^{(1)}$ and $\mathcal{E}_{\overline{\boldsymbol{\sigma}}}^{(1)}$ are reasonably close to the expected value of $2 / 3$. The convergence rates for the fluid variables of approximately 0.87 are somewhat less than the expected value of 1 for an isolated fluid calculation, but this is not unexpected here given that the fluid solution is strongly driven by the shocks in the solid which are converging at a rate 

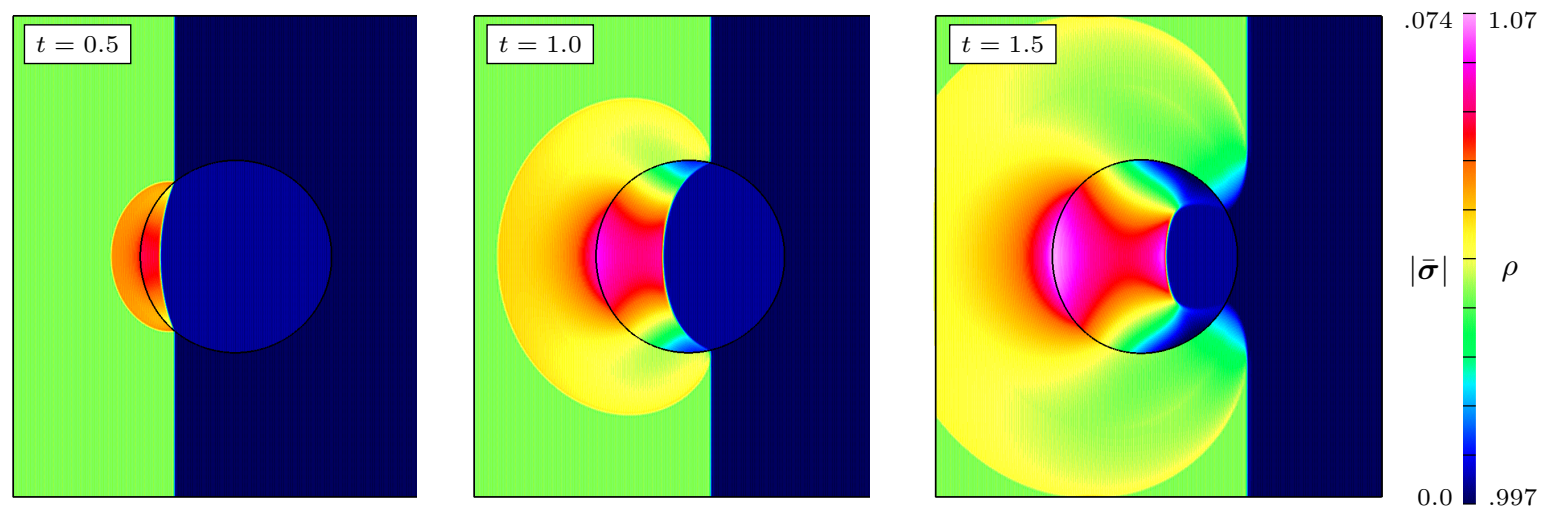

Figure 26: An elastic shock hitting a fluid filled deformable cylinder showing the density, $\rho$, in the fluid domain and the norm of the solid stress, $|\overline{\boldsymbol{\sigma}}|$, in the solid domain. Results are shown for the light solid, $\rho_{r}=0.1$, at times $t=0.5,1.0$ and 1.5 .
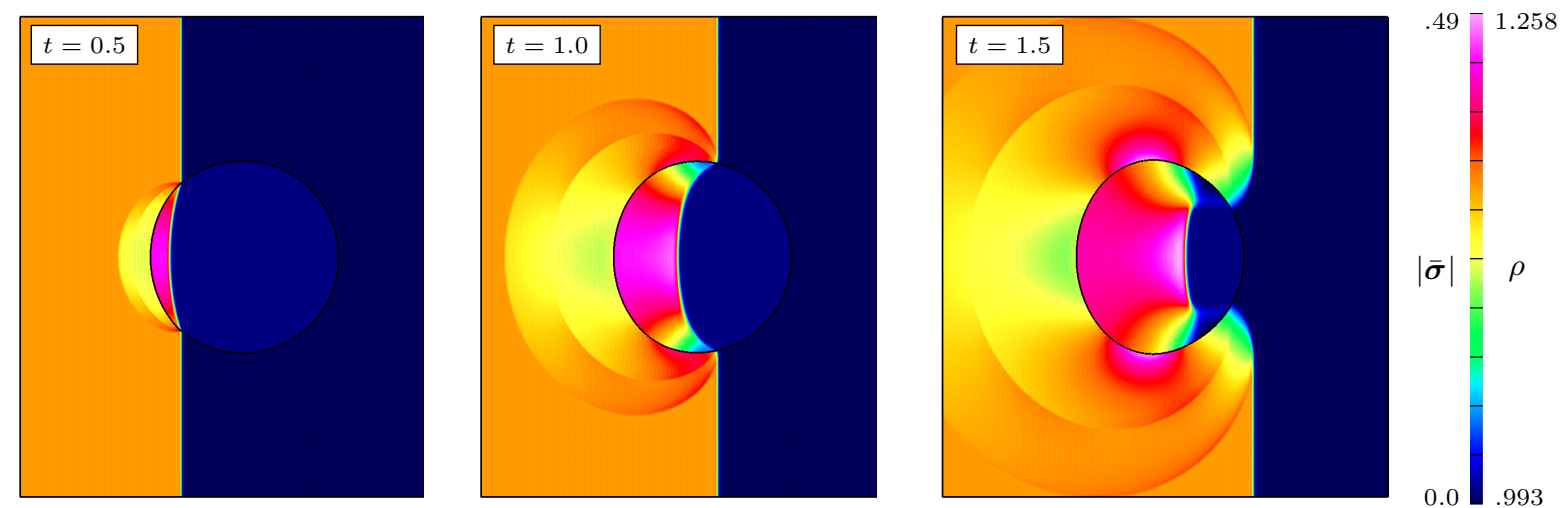

Figure 27: An elastic shock hitting a fluid filled deformable cylinder showing the density, $\rho$, in the fluid domain and the stress-norm $|\overline{\boldsymbol{\sigma}}|$, in the solid domain. Results are shown for the medium solid, $\rho_{r}=1.0$, at times $t=0.5,1.0$ and 1.5 .
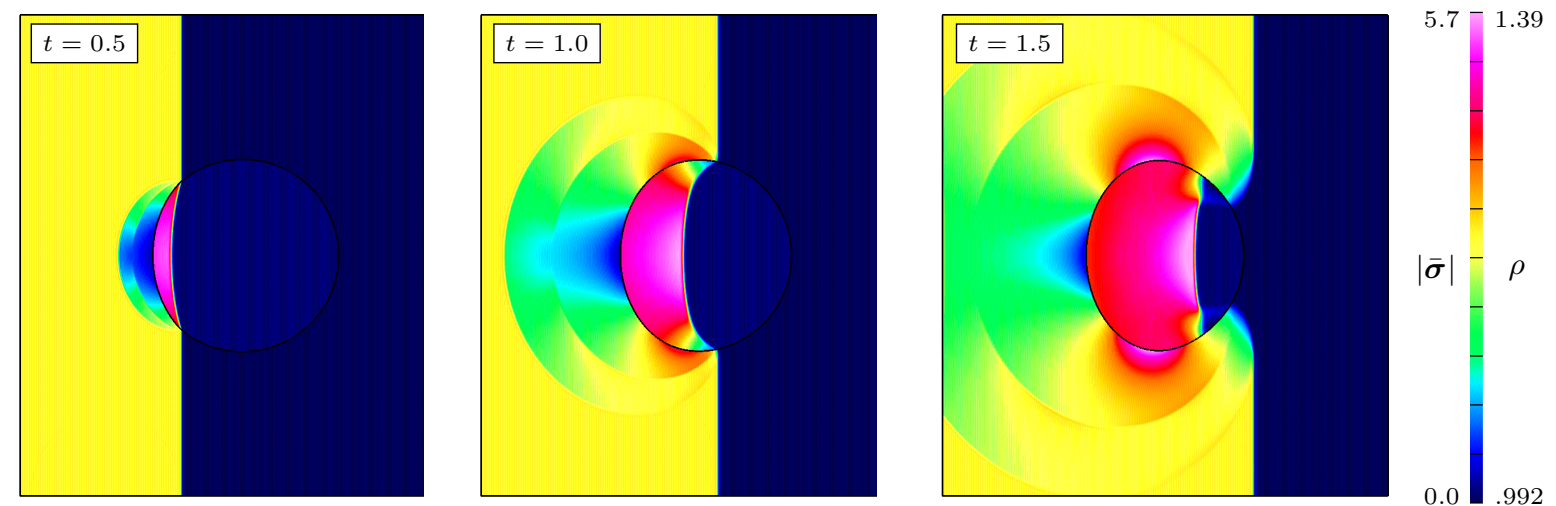

Figure 28: An elastic shock hitting a fluid filled deformable cylinder showing the density, $\rho$, in the fluid domain and the stress-norm $|\overline{\boldsymbol{\sigma}}|$, in the solid domain. Results are shown for the heavy solid, $\rho_{r}=10$, at times $t=0.5,1.0$ and 1.5 .

near 0.7 .

\subsection{A shock impacting two deformable sticks}

In this section, we consider the simulation of a fluid shock impacting two deformable sticks as shown in Figure 31. The main purpose of this computation is to demonstrate the ability of the FSI-DCG approach to treat problems with large displacements while retaining high-quality grids. We recognize that the use of a linear elastic model for the solids in this simulation is somewhat questionable from a physical standpoint since this solid model does not properly treat large strains or rotations, but this does not detract from the main purpose of this calculation.

The geometry of the problem consists of the large rectangular domain $\mathcal{R}=[-5,20] \times[-10,10]$ with two embedded solid sticks. Each stick is a smoothed-polygon approximation to the rectangular domain of width 0.5 and height 4 . The lower stick is centered at $(0,-2.1)$ and defines the solid domain $\bar{\Omega}_{1} \approx[-.25, .25] \times$ 


\begin{tabular}{|l|c|c|c|c|c|c|c|c|c|c|c|c|}
\hline & \multicolumn{9}{|c|}{ Solid } & \multicolumn{7}{c|}{ Fluid } \\
\hline Grid & $\mathcal{E}_{\overline{\mathbf{u}}}^{(1)}$ & $\mathrm{r}$ & $\mathcal{E}_{\overline{\mathbf{v}}}^{(1)}$ & $\mathrm{r}$ & $\mathcal{E}_{\overline{\boldsymbol{\sigma}}}^{(1)}$ & $\mathrm{r}$ & $\mathcal{E}_{\rho}^{(1)}$ & $\mathrm{r}$ & $\mathcal{E}_{\mathbf{v}}^{(1)}$ & $\mathrm{r}$ & $\mathcal{E}_{T}^{(1)}$ & $\mathrm{r}$ \\
\hline $\mathcal{G}_{d c}^{(4)}$ & $1.7 \mathrm{e}-4$ & & $1.1 \mathrm{e}-3$ & & $1.3 \mathrm{e}-3$ & & $4.1 \mathrm{e}-3$ & & $2.3 \mathrm{e}-3$ & & $4.2 \mathrm{e}-3$ & \\
\hline $\mathcal{G}_{d c}^{(8)}$ & $7.9 \mathrm{e}-5$ & 2.1 & $6.9 \mathrm{e}-4$ & 1.6 & $7.9 \mathrm{e}-4$ & 1.6 & $2.2 \mathrm{e}-3$ & 1.8 & $1.3 \mathrm{e}-3$ & 1.8 & $2.3 \mathrm{e}-3$ & 1.8 \\
\hline $\mathcal{G}_{d c}^{(64)}$ & $8.3 \mathrm{e}-6$ & 9.5 & $1.5 \mathrm{e}-4$ & 4.5 & $1.8 \mathrm{e}-4$ & 4.3 & $3.6 \mathrm{e}-4$ & 6.3 & $2.1 \mathrm{e}-4$ & 6.1 & $3.7 \mathrm{e}-4$ & 6.2 \\
\hline rate & 1.08 & & 0.72 & & 0.71 & & 0.88 & & 0.87 & & 0.88 & \\
\hline
\end{tabular}

Figure 29: Estimated $L_{1}$-norm errors and converge rates for the elastic shock hitting a fluid cavity, medium-density solid, $\rho_{r}=1.0$. Note that the grid spacing on the finest grid is 8 times smaller than the previous resolution.

$[-4.1,-.1]$. The upper stick is centered at $(0,2.1)$ and defines the domain $\bar{\Omega}_{2} \approx[-.25, .25] \times[.1,4.1]$. The fluid domain at time zero is then $\Omega(0)=\mathcal{R}-\bar{\Omega}_{1}-\bar{\Omega}_{2}$. Let $\mathcal{G}_{d s}^{(j)}$ denote the composite grid for this geometry with $\Delta s^{(j)}=1 /(10 j)$, approximately. Figure 30 shows closeups of the grid $\mathcal{G}_{d s}^{(4)}$ at three different times during the calculation. The grids next to the interface are hyperbolic grids with normal width $5 \Delta s^{(j)}$ for the fluid domain and width $3 \Delta s^{(j)}$ for the solid domains. The grids in the solid are shown adjusted for the displacement. Only the two green fluid-interface grids deform over time.

The initial conditions in the fluid correspond to a planar shock, initially at $x=-1$, traveling to the right with a shock Mach number equal to 2 . The state ahead of the shock is given by $\left[\rho, v_{1}, v_{2}, p\right]=[1.4,0,0 ., 1]$ and the state behind the shock by $[3.73333,1.25,0 ., 4.5]$. The ratio of specific heats in the fluid is $\gamma=1.4$. The material properties of the lower stick are $\bar{\rho}=\mu=\lambda=25$ and those of the (lighter) upper stick are $\bar{\rho}=\mu=\lambda=10$. The boundary conditions on the rectangular fluid domain are supersonic inflow on the left side (all variables given by the state behind the shock), slip walls on the top and bottom, and supersonic outflow on the right (all variables extrapolated).
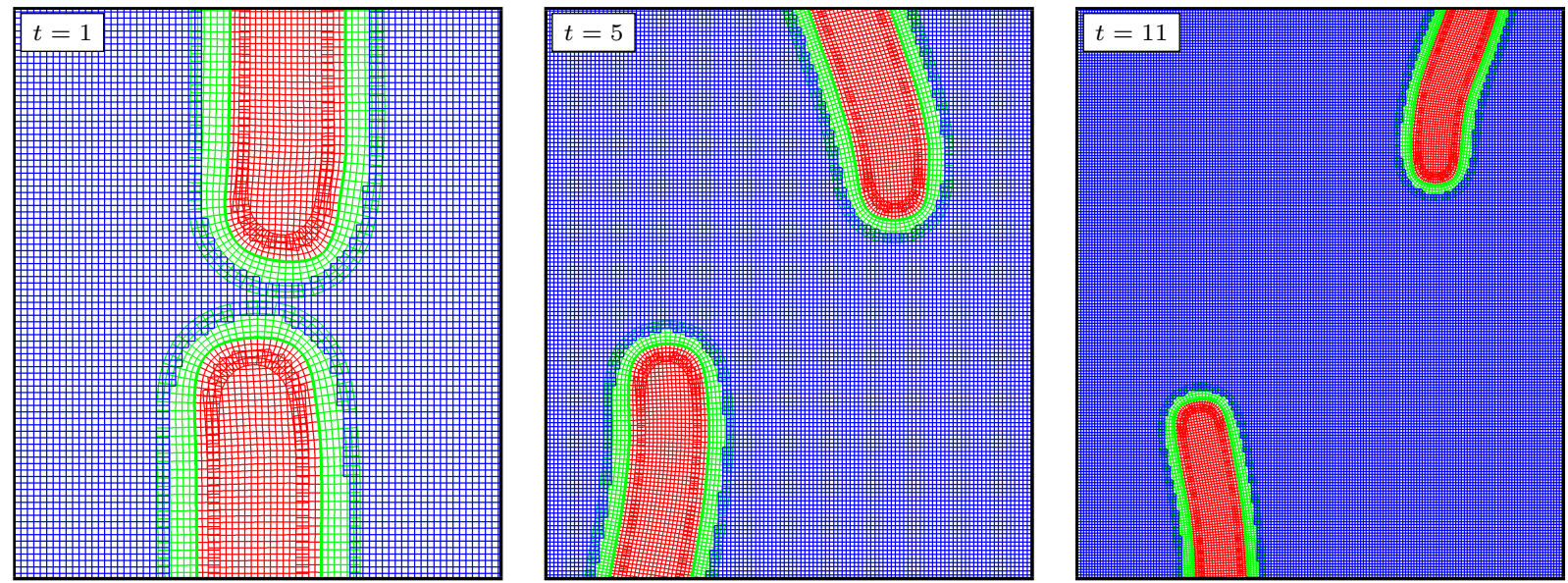

Figure 30: Closeup of the composite grids $\mathcal{G}_{d s}^{(4)}$ for the deformable sticks at times $t=1,5$ and 11 . The two deformable sticks move apart after being impacted by a fluid shock. The green curvilinear grids next to the interface are regenerated at each time step with the hyperbolic grid generator. The red grids in the solid domains are shown adjusted for the displacement. The grids remain smooth and of high quality throughout the simulation.

Figure 31 shows the solution of the FSI problem at different times from the computation using the grid $\mathcal{G}_{d s}^{(8)}$. Note that the region shown in the figures has been cropped from the full rectangular domain $\mathcal{R}$. The behavior of the fluid is displayed as numerically generated schlieren images. These gray-scale images are computed from the magnitude of the gradient in the fluid density, and they highlight shocks and contact discontinuities in the flow (see [22] for a definition of the numerical schlieren function). The plots also display shaded contours of the horizontal component of the velocity, $\bar{v}_{1}$, in the solid domains (illustrating which portions of the sticks are moving faster horizontally relative to other portions). The sticks are accelerated after being impacted by the shock, and subsequently deform and rotate as they propagate downstream. When the fluid shock first impacts the left edge of a stick it generates an elastic wave in the solid which moves from left to right through the stick. This wave subsequently reflects off the right side of the stick and at the same time creates a transmitted shock wave in the fluid. This elastic wave continues to bounce back and forth within the solid, and each time it hits a fluid-solid interface it generates a new wave in the fluid. A sequence of these waves in the fluid is particularly evident to the left of the lower stick in the solution at 
time $t=3$ of Figure 31. By the final time shown in the figure $(t=11)$, the incident shock in the fluid has traveled off the view and reflected shocks from the slip walls at the top and bottom of the fluid domain are visible. A complex flow field in the vicinity of the sticks is seen consisting of multiple reflected shocks and fluid roll-ups.
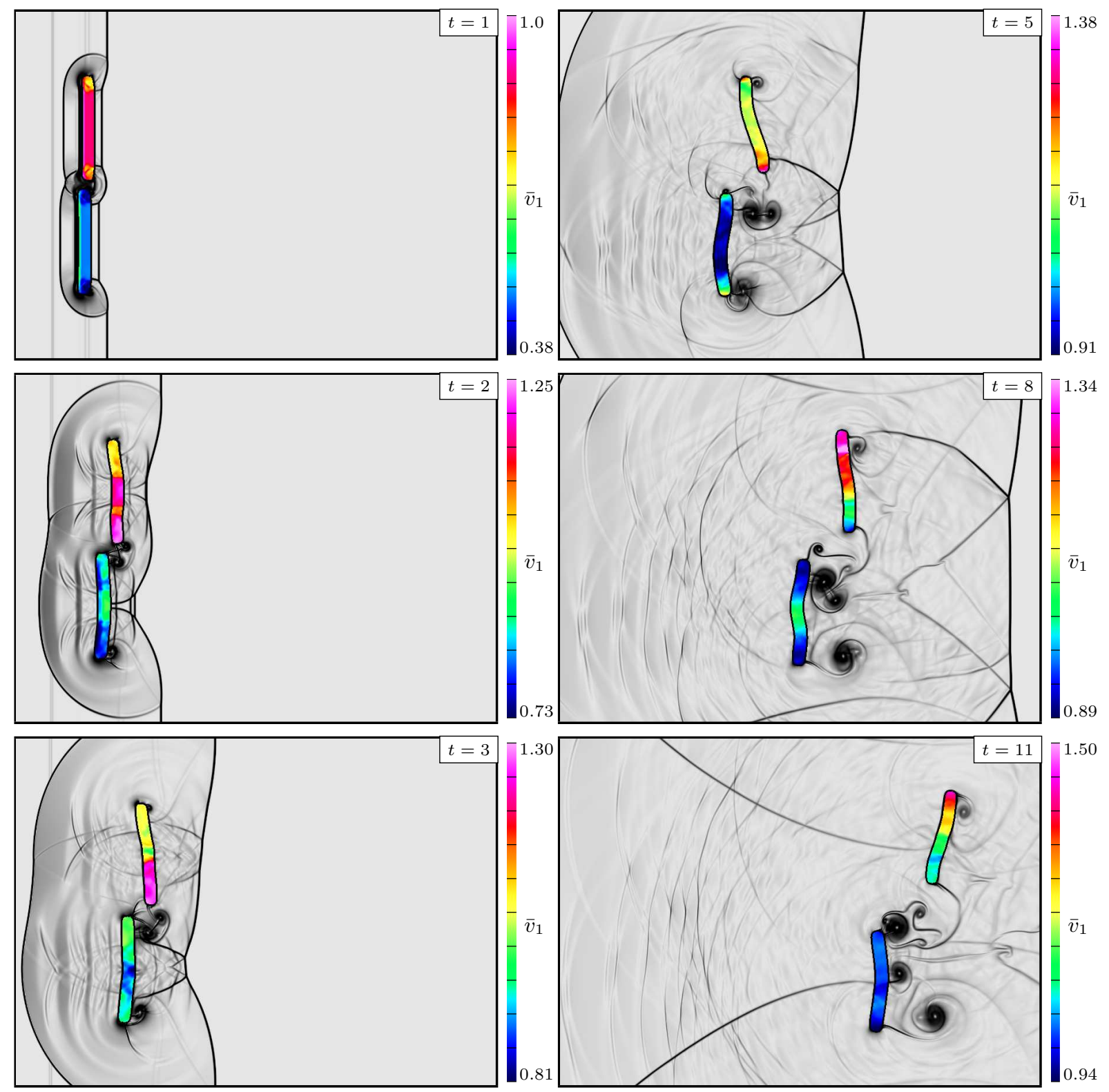

Figure 31: A shock hitting two elastic sticks showing the solution at times $t=1,2,3,5,8$ and 11 . The solution in the fluid domain is shown as a numerical schlieren image while the horizontal velocity $\bar{v}_{1}$ is shown in the solids. The solution was computed on grid $\mathcal{G}_{d s}^{(8)}$ which has 3.3 million grid points. Note that the bounds on the contour levels change with time.

The performance of the FSI-DCG scheme for the computation shown in Figure 31 is presented in Figure 32. The largest percentage of the total run time (73\%) was spent in advancing the fluid equations, as expected, since the majority of grid points belong to the fluid grids. The time spent advancing the solution in solid domains was less than $1 \%$. In terms of CPU time per grid point in the respective domains, the cost to advance the solution in the solid domains, $1.3 \mu \mathrm{s} / \mathrm{step}$, was about $45 \%$ of the cost to advance the solution in the fluid domain, $2.9 \mu \mathrm{s} / \mathrm{step}$ (including grid generation). About $23 \%$ of the time was spent moving and regenerating the grids in the fluid domain. We note that significant optimizations could be made to the grid generation stage but we leave this to future work.

Figure 33 compares the solution from simulations on a coarse grid $\mathcal{G}_{d s}^{(4)}\left(0.83 \times 10^{6}\right.$ grid points), a medium resolution grid $\mathcal{G}_{d s}^{(8)}\left(3.3 \times 10^{6}\right.$ grid points $)$ and fine grid $\mathcal{G}_{d s}^{(16)}\left(13.2 \times 10^{6}\right.$ grid points). The finer grids produce 


\begin{tabular}{|r|r|r|}
\cline { 2 - 3 } & \multicolumn{2}{|c|}{ Deforming Sticks } \\
\cline { 2 - 3 } advance fluid (3.2M pts) & s/step & \% CPU \\
\cline { 2 - 3 } advance solids (.03M pts) & 6.8 & 73 \\
grid generation & .04 & .4 \\
interfaces & 2.2 & 23 \\
other & .07 & .7 \\
total & .29 & 2.9 \\
\cline { 2 - 3 } & 9.4 & 100 \\
\hline
\end{tabular}

Figure 32: Computational performance of FSI-DCG scheme for the deforming stick problem shown in Figure 31\} CPU time (in seconds) for various parts of the algorithm and their percentages of the total CPU time per step. The total number of grid points was approximately 3.3 million with the majority belonging to the fluid domain. The computation was run on a single core of a 4-core Xeon $2.3 \mathrm{GHz}$ processor.

increasingly sharper and more detailed features but the the overall structure of the flow compares very well between the different runs.

\section{Conclusions}

We have described a mixed Eulerian-Lagrangian technique for the solution of fluid-structure interaction (FSI) problems. This general and flexible approach uses deforming composite grids (DCG) to represent fluidsolid interfaces with boundary-conforming grids that track the interfaces as they evolve. The interface grids move through static background grids and remain smooth and of high quality even under large displacements and rotations of the solid domains. The FSI-DCG approach was developed and verified for a system consisting of inviscid compressible fluids described by the Euler equations coupled to solids modeled by the (linear) elastic wave equation. At an interface, the normal components of the fluid and solid velocities must match, along with the fluid and solid tractions. An interface approximation based on the solution to a fluid-solid Riemann (FSR) problem has been developed to couple the solutions at an interface. This FSR approximation is accurate and stable for a wide range of regimes, including the difficult case of light solids when the standard velocity-from-solid/stress-from-fluid approximation fails.

The FSI-DCG time-stepping scheme and interface approximations have been described in detail and carefully validated for the one-dimensional elastic piston problem. The extension of the scheme to problems in two space dimensions has also been described. The two-dimensional FSI-DCG scheme has been carefully verified on three problems with known solutions: the two-dimensional elastic-piston problem, the superseismic-shock problem and a deforming-diffuser problem. For the deforming-diffuser problem, which has a smooth solution, and the smoothly receding elastic piston, the scheme was shown to be second-order accurate in the max-norm. For the problems which contain discontinuities, the expected rates of convergence were also obtained. Simulations were performed of an elastic shock wave impacting a fluid-filled cavity to demonstrate that the multi-dimensional algorithm remains stable even for light solids. A self-convergence grid refinement study for this case showed the solutions to be converging at the expected rates. A final example of a shock impacting two deformable sticks illustrated the application of the method to a problem where the solids undergo large translational motions through the fluid domain.

There are many avenues to follow in future work. The FSI-DCG approach described here can be extended to other fluid models including viscous fluids and incompressible fluids as well as more general solid models that can treat large rotations and deformations. In addition we will extend the current numerical framework to three space dimensions, to handle non-matching interface grids and adaptive mesh refinement, as well as to run in parallel on distributed memory computers.

\section{Appendix A. Solution of the receding elastic-piston problem}

A smooth exact solution to the elastic-piston problem can be derived for the case of a receding piston. This is a good test problem for showing the accuracy of our numerical scheme when the solution is smooth. The $x$ - $t$ diagram was shown previously in Figure 1. At $t=0$, the fluid is at rest with constant initial conditions $\rho(x, 0)=\rho_{0}, v(x, 0)=0, p(x, 0)=p_{0}$ and $a(x, 0)=a_{0}=\sqrt{\gamma p_{0} / \rho_{0}}$. Assuming a given interface motion, $x=G(t)$, the solution in the fluid region $G(t)<x<a_{0} t$ is given by the method of characteristics,

$$
v(x, t)=\dot{G}(\tau(x, t)), \quad \frac{a(x, t)}{a_{0}}=1+\frac{\gamma-1}{2}\left(\frac{v(x, t)}{a_{0}}\right), \quad \frac{p(x, t)}{p_{0}}=\left(\frac{\rho(x, t)}{\rho_{0}}\right)^{\gamma}=\left(\frac{a(x, t)}{a_{0}}\right)^{2 \gamma /(\gamma-1)},
$$



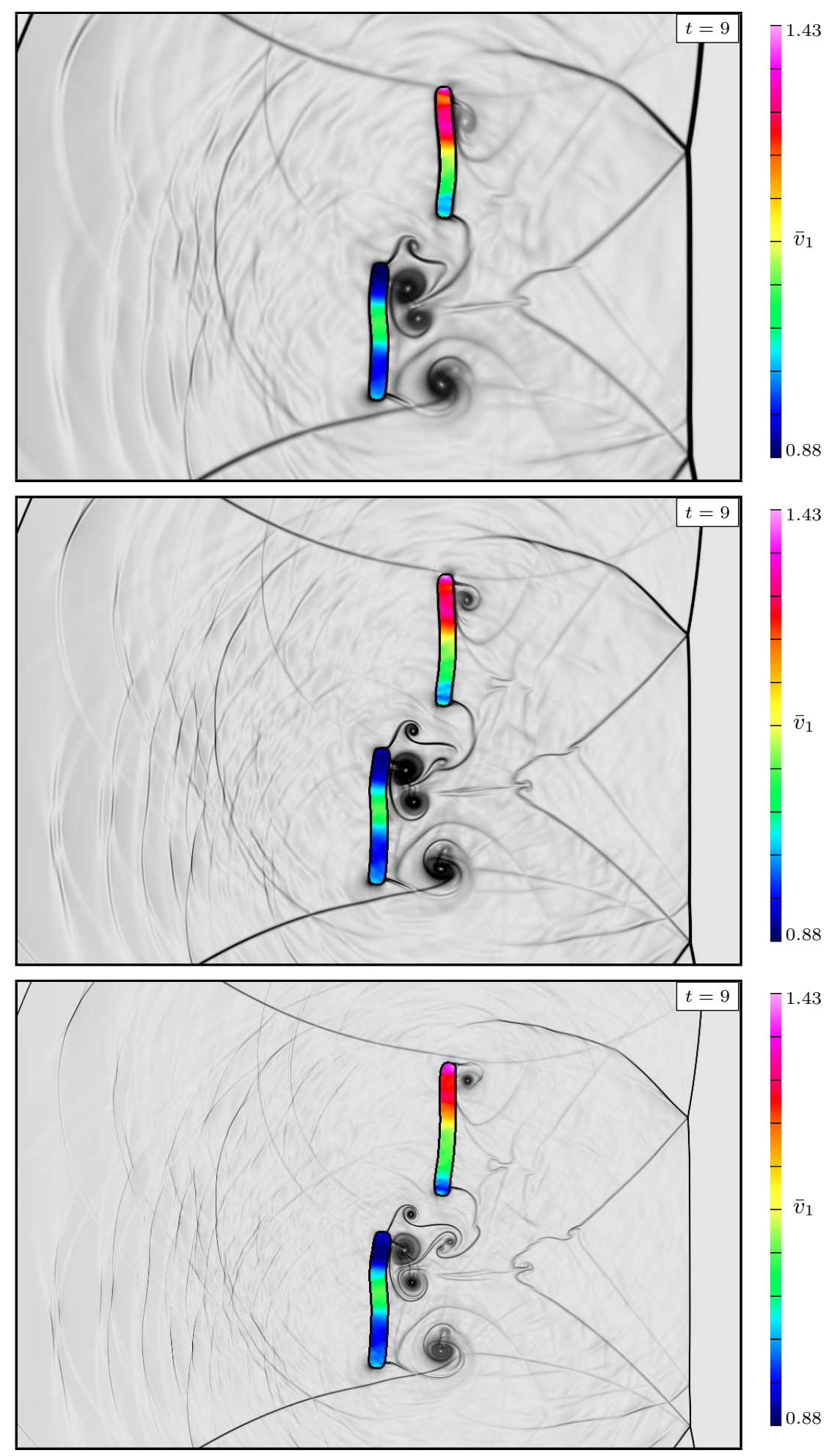

Figure 33: A shock hitting two elastic sticks. A comparision of the solution at $t=9$ on the coarse grid $\mathcal{G}_{d s}^{(4)}$ (top) medium resolution grid $\mathcal{G}_{d s}^{(8)}$ (middle) and fine grid $\mathcal{G}_{d s}^{(16)}$ (bottom).

where $\tau=\tau(x, t)$ satisfies

$$
x-G(\tau)=\left[a_{0}+\frac{\gamma+1}{2} \dot{G}(\tau)\right](t-\tau) .
$$

The solution in the fluid region for $x>a_{0} t$ is simply $\rho(x, t)=\rho_{0}, v(x, t)=0$ and $p(x, t)=p_{0}$. (For further details see the discussion in [22].) We have assumed that the interface moves so that no shocks form which 
will be the case if $\dot{G} \leq 0$ and $\ddot{G} \leq 0$.

The solution to the elasticity equations with initial conditions $\bar{u}(\bar{x}, 0)=\bar{u}_{0}(\bar{x})$ and $\partial_{t} \bar{u}(\bar{x}, 0)=\bar{v}_{0}(\bar{x})$ and boundary condition $\bar{u}(0, t)=G(t)$ follows from the d'Alembert solution,

$$
\begin{aligned}
\bar{u}(\bar{x}, t) & =f\left(\bar{x}-c_{p} t\right)+g\left(\bar{x}+c_{p} t\right), \\
f(\xi) & =\frac{1}{2}\left[\bar{u}_{0}(\xi)-c_{p}^{-1} \int_{0}^{\xi} \bar{v}_{0}(s) d s\right] \\
g(\xi) & = \begin{cases}\frac{1}{2}\left[\bar{u}_{0}(\xi)+c_{p}^{-1} \int_{0}^{\xi} \bar{v}_{0}(s) d s\right] & \text { for } \xi<0, \\
G\left(\xi / c_{p}\right)-f(-\xi) & \text { for } \xi>0 .\end{cases}
\end{aligned}
$$

By imposing the interface traction condition, $\bar{\rho} c_{p}^{2} \partial_{\bar{x}} \bar{u}(0, t)=-p(G(t), t)$ we arrive at the following equation for $\dot{G}(t)$,

$$
\frac{p_{0}}{\bar{\rho} c_{p}^{2}}\left[1+\frac{\gamma-1}{2 a_{0}} \dot{G}(t)\right]^{2 \gamma /(\gamma-1)}+\frac{\dot{G}(t)}{c_{p}}=-\left[\bar{u}_{0}^{\prime}\left(-c_{p} t\right)-\frac{1}{c_{p}} \bar{v}_{0}\left(-c_{p} t\right)\right], \text { for } t>0 .
$$

Given any appropriate initial conditions for the fluid and solid, (A.6) can be solved as a nonlinear ODE to determine $G(t)$. We proceed, however, in a different manner and first choose a form for $G(t)$ and then determine initial conditions $\bar{u}_{0}(\bar{x})$ and $\bar{v}_{0}(\bar{x})$ in the solid to be consistent with (A.6). In particular, we choose

$$
G(t)=-\frac{G_{a}}{q} t^{q}
$$

for some positive integer $q$ and amplitude parameter $G_{a}$. Given $G(t)$, there are many possible choices for $\bar{u}_{0}(\bar{x})$ and $\bar{v}_{0}(\bar{x})$ that will satisfy (A.6). Since we are looking for a smooth exact solution, it is natural to require the initial conditions to satisfy the fluid-solid interface conditions (which they do not necessarily need to satisfy). This gives the choice

$$
\bar{u}_{0}(\bar{x})=-\frac{p_{0}}{\bar{\rho}_{0} c_{p}^{2}} \int_{0}^{\bar{x}}\left[1+\frac{\gamma-1}{2 a_{0}} \dot{G}\left(-s / c_{p}\right)\right]^{2 \gamma /(\gamma-1)} d s, \quad \bar{v}_{0}(\bar{x})=\dot{G}\left(-\bar{x} / c_{p}\right), \quad \text { for } \bar{x}<0 .
$$

In summary, given an interface motion $G(t)$ from (A.7), we choose initial conditions in the solid from (A.8), and then the solution in the solid is given by (A.3)-(A.5) and the solution in the fluid is given by (A.1) $-($ A.2) .

\section{Appendix B. Solution of the elastic-piston shock-tube problem}

An exact solution to the elastic-piston problem can be derived for the situation of a fluid shock hitting an initially motionless piston. This solution is used in Section 4.3 .2 to evaluate our numerical scheme. The $x-t$ diagram for the problem is shown in Figure B.34. The solution to this problem is a simple extension of the fluid-solid Riemann solution given in Section 4.1. An incident shock moves from right to left and impacts the solid at $t=t_{i}$. The shock reflects, causes the interface to move, and in turn sets up an elastic shock wave in the solid. The initial conditions are

$$
\begin{aligned}
{[\bar{v}(\bar{x}, 0), \bar{\sigma}(\bar{x}, 0)] } & =[0,0], \quad \text { for } \bar{x}<0, \\
{[\rho(x, 0), v(x, 0), p(x, 0)] } & = \begin{cases}{\left[\rho_{0}, 0, p_{0}\right],} & \text { for } 0<x<x_{0}, \\
{\left[\rho_{1}, v_{1}, p_{1}\right],} & \text { for } x>x_{0},\end{cases}
\end{aligned}
$$

where the initial fluid states are chosen to correspond to a shock moving to the left with speed $S_{0}$. At time $t=t_{i}=x_{0} / S_{0}$ the incident shock impacts the solid. The solution at this time defines a fluid-solid Riemann problem with the constant solid state $\left[\bar{v}_{0}=0, \bar{\sigma}_{0}=0\right]$ next to the fluid state $\left[\rho_{1}, v_{1}, p_{1}\right]$. The solution to this problem is given in Section 4.1.2.

\section{Appendix C. Solution of the superseismic-shock problem}

Here we provide the analytic solution to the superseismic shock problem discussed in Section 6.2. The structure of the solution was shown earlier in Figure 17. (Note that there are also solutions where the s-wave 


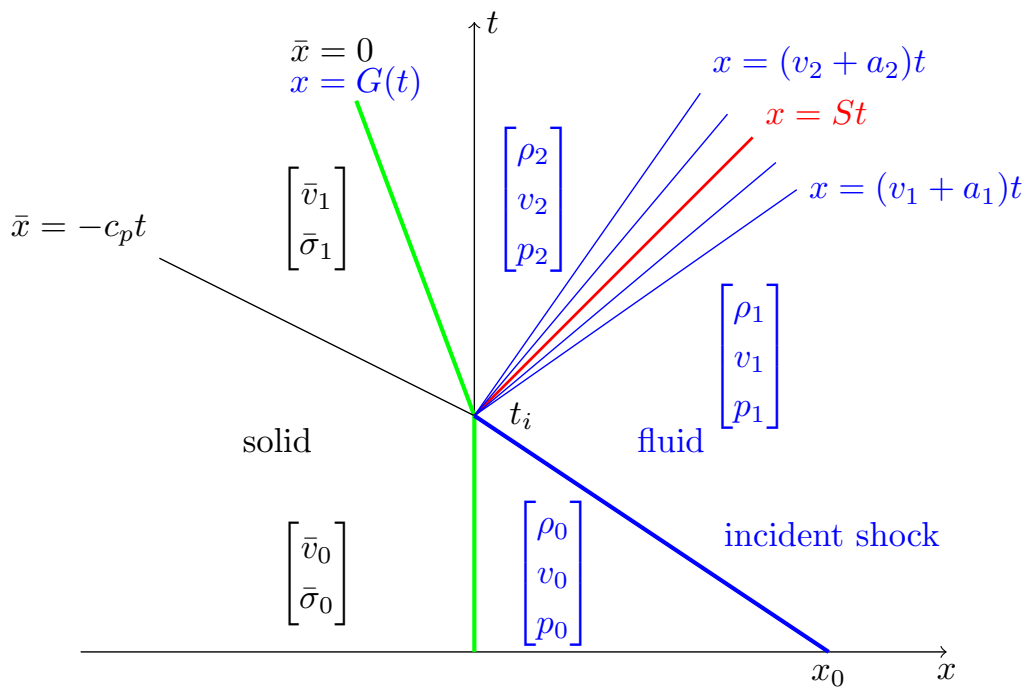

Figure B.34: The $x-t$ diagram for the elastic shock tube. An incident shock moves from right to left and impacts the solid at $t=t_{i}$. The impact causes the interface to move and sets up an elastic shock wave in the solid. The reflected wave in the fluid may be a shock or an expansion fan.

leads the p-wave and where the s- and p-waves lie in the lower right-half plane.) The input parameters to the problem are the solid material parameters $\bar{\rho}, \mu$ and $\lambda$, the speed of the structure $S$, the upstream fluid state $\mathbf{w}^{0}=\left[\rho^{0}, v_{1}^{0}, 0, p^{0}\right]$ and the ratio of specific heats in the fluid $\gamma$. Assume, to begin with, that the shock angle $\xi$ is known (even though $\xi$ will normally have to be computed as a solution to the equations given below). Define the angle $\beta=\pi / 2-\xi$, the upstream speed of sound $a_{0}=\sqrt{\gamma p^{0} / \rho^{0}}$ and the upstream Mach number $M_{0}=\left(S-v_{1}^{0}\right) / a_{0}$. The post-shock fluid state is given by the oblique shock conditions (see for example Liepmann and Roshko [44]),

$$
\begin{aligned}
\frac{p^{1}-p^{0}}{p^{0}} & =\frac{2 \gamma}{\gamma+1}\left[M_{0}^{2} \sin ^{2} \beta-1\right], \quad \rho^{1}=\rho^{0} \frac{(\gamma+1) M_{0}^{2} \sin ^{2} \beta}{(\gamma-1) M_{0}^{2} \sin ^{2} \beta+2}, \\
\mathbf{v}_{1}^{1} & =\left[\begin{array}{l}
v_{1}^{1} \\
v_{2}^{1}
\end{array}\right]=\left[\begin{array}{l}
S \\
0
\end{array}\right]+\left(v_{1}^{0}-S\right) \frac{\cos \beta}{\cos (\beta-\theta)}\left[\begin{array}{c}
\cos \theta \\
\sin \theta
\end{array}\right],
\end{aligned}
$$

where the angle of deflection of the interface, $\theta$, is related to $\xi$ by

$$
\tan \theta=2 \cot \beta\left\{\frac{M_{0}^{2} \sin ^{2} \beta-1}{M_{0}^{2}(\gamma+\cos 2 \beta)+2}\right\} .
$$

Define the wave-vector, $\boldsymbol{\kappa}$, eigenvector, a, and angle $\eta$ for each of the two traveling waves in the solid,

$$
\begin{aligned}
& \kappa_{1}^{p}=\cos \eta_{p}=c_{p} / S, \quad \kappa_{2}^{p}=-\sin \left(\eta_{p}\right), \quad \kappa_{1}^{s}=\cos \eta_{s}=c_{s} / S, \quad \kappa_{2}^{s}=-\sin \left(\eta_{s}\right), \\
& \boldsymbol{\kappa}^{p}=\left[\begin{array}{l}
\kappa_{1}^{p} \\
\kappa_{2}^{p}
\end{array}\right], \quad \boldsymbol{\kappa}^{s}=\left[\begin{array}{l}
\kappa_{1}^{s} \\
\kappa_{2}^{s}
\end{array}\right], \quad \mathbf{a}^{p}=\left[\begin{array}{l}
\kappa_{1}^{p} \\
\kappa_{2}^{p}
\end{array}\right], \quad \mathbf{a}^{s}=\left[\begin{array}{c}
-\kappa_{2}^{s} \\
\kappa_{1}^{s}
\end{array}\right] .
\end{aligned}
$$

In terms of the wave strengths $\alpha_{p}$ and $\alpha_{s}$ (yet to be determined), the displacements and velocities in the solid states $\overline{\mathbf{w}}^{0}, \overline{\mathbf{w}}^{1}$ and $\overline{\mathbf{w}}^{2}$ are

$$
\begin{aligned}
& \overline{\mathbf{u}}^{0}=\mathbf{0}, \quad \overline{\mathbf{u}}^{1}=-\alpha_{p}\left(\boldsymbol{\kappa}^{p} \cdot \overline{\mathbf{x}}-c_{p} t\right) \mathbf{a}^{p}, \quad \overline{\mathbf{u}}^{2}=\overline{\mathbf{u}}^{1}-\alpha_{s}\left(\boldsymbol{\kappa}^{s} \cdot \overline{\mathbf{x}}-c_{s} t\right) \mathbf{a}^{s}, \\
& \overline{\mathbf{v}}^{0}=\mathbf{0}, \quad \overline{\mathbf{v}}^{1}=\alpha_{p} c_{p} \mathbf{a}^{p}, \quad \overline{\mathbf{v}}^{2}=\overline{\mathbf{v}}^{1}+\alpha_{s} c_{s} \mathbf{a}^{s} .
\end{aligned}
$$

The three stress states of the solid can be written as

$$
\overline{\boldsymbol{\sigma}}^{0}=\mathbf{0}, \quad \overline{\boldsymbol{\sigma}}^{1}=\overline{\boldsymbol{\sigma}}^{p}, \quad \overline{\boldsymbol{\sigma}}^{2}=\overline{\boldsymbol{\sigma}}^{1}+\overline{\boldsymbol{\sigma}}^{s},
$$

where the components of the tensors $\overline{\boldsymbol{\sigma}}^{p}$ and $\overline{\boldsymbol{\sigma}}^{s}$ are given by

$$
\bar{\sigma}_{i j}^{p}=\left[\begin{array}{cc}
\lambda+2 \mu\left(\kappa_{1}^{p}\right)^{2} & 2 \mu \kappa_{1}^{p} \kappa_{2}^{p} \\
2 \mu \kappa_{1}^{p} \kappa_{2}^{p} & \lambda+2 \mu\left(\kappa_{2}^{p}\right)^{2}
\end{array}\right] \quad\left(-\alpha_{p}\right), \quad \bar{\sigma}_{i j}^{s}=\left[\begin{array}{cc}
-2 \mu \kappa_{1}^{s} \kappa_{2}^{s} & \mu\left(\left(\kappa_{1}^{s}\right)^{2}-\left(\kappa_{2}^{s}\right)^{2}\right) \\
\mu\left(\left(\kappa_{1}^{s}\right)^{2}-\left(\kappa_{2}^{s}\right)^{2}\right) & 2 \mu \kappa_{1}^{s} \kappa_{2}^{s}
\end{array}\right]\left(-\alpha_{s}\right) .
$$


The wave strengths $\alpha_{p}$ and $\alpha_{s}$ are then solutions to traction interface condition,

$$
\overline{\boldsymbol{\sigma}}^{2} \overline{\mathbf{n}}=-\left(p^{1}-p^{0}\right) \mathbf{n}
$$

where the normal vectors used for the solid and fluid are

$$
\overline{\mathbf{n}}=\left[\begin{array}{l}
0 \\
1
\end{array}\right] \text { and } \mathbf{n}=\left[\begin{array}{c}
-\sin \theta \\
\cos \theta
\end{array}\right]
$$

Note that the normal for the solid is taken from the reference state $\overline{\mathbf{x}}$ as is often done for linear elasticity. Given $\alpha_{p}$ and $\alpha_{s}$ from the solution to (C.10) we then know the solid states. Supposing the value of $\xi$ we started with was correct, then the velocity interface condition

$$
\tan \theta=\frac{\bar{v}_{2}^{2}}{\bar{v}_{1}^{2}}
$$

will also be satisfied. Otherwise one can use a root-finding procedure to determine a value for $\xi$ that satisfies the above relationships.

\section{References}

[1] J. Donea, S. Giuliani, J. P. Halluex, An arbitrary Lagrangian-Eulerian finite element method for transient dynamic fluid-structure interactions, Comput. Method. Appl. M. 33 (1982) 689-723.

[2] R. Löhner, C. Yang, J. D. Baum, H. Luo, D. Pelessone, C. M. Charman, The numerical simulation of strongly unsteady flow with hundreds of moving bodies, Int. J. Numer. Meth. Fl. 31 (1999) 113-120.

[3] E. Kuhl, S. Hulshoff, R. de Borst, An arbitray Lagrangian Eulerian finite-element approach for fluidstructure interaction phenomena, Int. J. Numer. Meth. Eng. 57 (2003) 117-142.

[4] H. T. Ahn, Y. Kallinderis, Strongly coupled flow/structure interactions with a geometrically conservative ALE scheme on general hybrid meshes, J. Comput. Phys. 219 (2006) 671-696.

[5] I. Borazjani, L. Ge, F. Sotiropolous, Curvilinear immersed boundary method for simulating fluid structure interaction with complex 3D rigid bodies, J. Comput. Phys. 227 (2008) 7587-7620.

[6] J. T. Grétarsson, N. Kwatra, R. Fedkiw, Numerically stable fluid-structure interactions between compressible flow and solid structures, J. Comput. Phys. 230 (2011) $3062-3084$.

[7] F. Cirak, R. Deiterding, S. P. Mauch, Large-scale fluid-structure interaction simulation of viscoplastic and fracturing thin-shells subjected to shocks and detonations, Computers \& Structures 85 (2007) 1049 - 1065. Fourth MIT Conference on Computational Fluid and Solid Mechanics.

[8] M. Arienti, P. Hung, E. Morano, J. E. Shepherd, A level set approach to Eulerian-Lagrangian coupling, J. Comput. Phys. 185 (2003) $213-251$.

[9] P. T. Barton, B. Obadia, D. Drikakis, A conservative level-set based method for compressible solid/fluid problems on fixed grids, J. Comput. Phys. In Press, Accepted Manuscript (2011) -.

[10] J. Glimm, J. W. Grove, X. L. Li, D. C. Tan, Robust computational algorithms for dynamic interface tracking in three dimensions, SIAM J. Sci. Comput. 21 (1999) 2240-2256.

[11] J. Du, B. Fix, J. Glimm, X. Jia, X. Li, Y. Li, L. Wu, A simple package for front tracking, J. Comput. Phys. 213 (2006) 613-628.

[12] R. van Loon, P. D. Anderson, F. N. van de Vosse, S. J. Sherwin, Comparison of various fluid-structure interaction methods for deformable bodies, Comput. Struct. 85 (2007) 833 - 843. Fourth MIT Conference on Computational Fluid and Solid Mechanics.

[13] T. E. Tezduyar, S. Sathe, R. Keedy, K. Stein, Space-time finite element techniques for computation of fluid-structure interactions, Comput. Method. Appl. M. 195 (2006) 2002 - 2027. Fluid-Structure Interaction. 
[14] M. Schäfer, I. Teschauer, Numerical simulation of coupled fluid-solid problems, Comput. Method. Appl. M. 190 (2001) 3645-3667.

[15] G. H. Miller, P. Colella, A conservative three-dimensional Eulerian method for coupled solid-fluid shock capturing, J. Comput. Phys. 183 (2002) 26-82.

[16] L. C. Wilcox, G. Stadler, C. Burstedde, O. Ghattas, A high-order discontinuous galerkin method for wave propagation through coupled elastic-acoustic media, J. Comput. Phys. 229 (2010) 9373 - 9396.

[17] C.-S. Yih, Fluid Mechanics, West River Press, 1977.

[18] W. D. Henshaw, A high-order accurate parallel solver for Maxwell's equations on overlapping grids, SIAM J. Sci. Comput. 28 (2006) 1730-1765.

[19] D. Appelö, J. W. Banks, W. D. Henshaw, D. W. Schwendeman, Numerical methods for solid mechanics on overlapping grids: Linear elasticity (2010). Submitted for publication.

[20] F. C. Dougherty, J.-H. Kuan, Transonic Store Separation Using a Three-Dimensional Chimera Grid Scheme, paper 89-0637, AIAA, 1989.

[21] R. Meakin, Moving Body Overset Grid Methods for Complete Aircraft Tiltrotor Simulations, paper 93-3350, AIAA, 1993.

[22] W. D. Henshaw, D. W. Schwendeman, Moving overlapping grids with adaptive mesh refinement for high-speed reactive and non-reactive flow, J. Comput. Phys. 216 (2006) 744-779.

[23] F. Zahle, J. Johansen, N. N. Sørensen, J. M. R. Graham, Wind Turbine Rotor-Tower Interaction Using an Incompressible Overset Grid Method, paper 2007-425, AIAA, 2007.

[24] W. M. Chan, Overset grid technology development at NASA Ames Research Center, Comput. Fl. 38 (2009) 496-503.

[25] D. D. J. Chandar, M. Damodaran, Numerical study of the free flight characteristics of a flapping wing in low Reynolds numbers, AIAA J. Aircraft 47 (2010) 141-150.

[26] W. D. Henshaw, K. K. Chand, A composite grid solver for conjugate heat transfer in fluid-structure systems, J. Comput. Phys. 228 (2009) 3708-3741.

[27] J. W. Banks, B. Sjögreen, A normal mode stability analysis of numerical interface conditions for fluid/structure interaction, Commun. Comput. Phys. 10 (2011) 279-304.

[28] G. B. Whitham, Linear and Nonlinear Waves, John Wiley and Sons, New York, 1974.

[29] W. D. Henshaw, D. W. Schwendeman, An adaptive numerical scheme for high-speed reactive flow on overlapping grids, J. Comput. Phys. 191 (2003) 420-447.

[30] W. J. Rider, Revisiting wall heating, J. Comput. Phys. 162 (2000) 395-410.

[31] J. W. Banks, T. Aslam, W. J. Rider, On sub-linear convergence for linearly degenerate waves in capturing schemes, J. Comput. Phys. 227 (2008) 6985-7002.

[32] W. D. Henshaw, D. W. Schwendeman, Parallel computation of three-dimensional flows using overlapping grids with adaptive mesh refinement, J. Comput. Phys. 227 (2008) 7469-7502.

[33] G. S. Chesshire, W. D. Henshaw, Composite overlapping meshes for the solution of partial differential equations, J. Comput. Phys. 90 (1990) 1-64.

[34] W. D. Henshaw, Ogen: An Overlapping Grid Generator for Overture, Research Report UCRL-MA132237, Lawrence Livermore National Laboratory, 1998.

[35] G. Starius, Constructing orthogonal curvilinear meshes by solving initial value problems, Numer. Math. 28 (1977) $25-48$.

[36] J. L. Steger, D. S. Chaussee, Generation of body fitted coordinates using hyperbolic partial differential equations, SIAM J. Sci. Comput. 1 (1980) 431-437. 
[37] W. M. Chan, J. L. Steger, Enhancements of a three-dimensional hyperbolic grid generation scheme, Appl. Math. Comput. 51 (1992) 181-205.

[38] W. D. Henshaw, The Overture Hyperbolic Grid Generator, User Guide, Version 1.0, Research Report UCRL-MA-134240, Lawrence Livermore National Laboratory, 1999.

[39] R. K. Jaiman, X. Jiao, P. H. Geubelle, E. Loth, Assessment of conservative load transfer for fluid-solid interface with non-matching meshes, Int. J. Numer. Meth. Fl. 64 (2005) 2014-2038.

[40] A. A. Kaufman, A. L. Levshin, Acoustic and Elastic Wave Fields in Geophysics, Elsevier, 2005.

[41] W. D. Henshaw, Mappings for Overture, A Description of the Mapping Class and Documentation for Many Useful Mappings, Research Report UCRL-MA-132239, Lawrence Livermore National Laboratory, 1998.

[42] G. Terrones, Fastest growing linear Rayleigh-Taylor modes at solid/fluid and solid/solid interfaces, Phys. Rev. E 71 (2005) 036306.

[43] J. J. L. Cela, A. R. Piriz, M. C. S. Moreno, N. A. Tahir, Numerical simulations of Rayleigh-Taylor instability in elastic solids, Lasers Part. Beams 24 (2006).

[44] H. W. Liepmann, A. Roshko, Elements of Gasdynamics, John Wiley and Sons, New York, 1966. 